\title{
Partially and fully frustrated coupled oscillators with random pinning fields
}

\author{
A C C Coolen $\dagger$ and C Pérez-Vicente $\ddagger$ \\ $\dagger$ Department of Mathematics, King's College London, The Strand, London \\ WC2R 2LS, UK \\ $\ddagger$ Departament de Fisica Fonamental, Facultat de Fisica, Universitat de \\ Barcelona, 08028 Barcelona, Spain \\ PACS numbers: $75.10 . \mathrm{Nr}, 05.20 .-\mathrm{y}, 64.60 . \mathrm{Cn}$ \\ E-mail: tcoolen@mth.kcl.ac.uk, conrad@ffn.ub.es
}

\begin{abstract}
We have studied two specific models of frustrated and disordered coupled Kuramoto oscillators, all driven with the same natural frequency, in the presence of random external pinning fields. Our models are structurally similar, but differ in their degree of bond frustration and in their finite size ground state properties (one has random ferro- and anti-ferromagnetic interactions; the other has random chiral interactions). We have calculated the equilibrium properties of both models in the thermodynamic limit using the replica method, with emphasis on the role played by symmetries of the pinning field distribution, leading to explicit predictions for observables, transitions, and phase diagrams. For absent pinning fields our two models are found to behave identically, but pinning fields (provided with appropriate statistical properties) break this symmetry. Simulation data lend satisfactory support to our theoretical predictions.
\end{abstract}

\section{Introduction}

The dynamics and the analysis of models of interacting oscillators have received an increasing amount of attention during the last few decades [1]. These models are excellent for studying synchronization phenomena and other features related to the temporal activity of populations of microscopic elements with limit cycle behaviour. They have been studied in many fields, including physics, chemistry and biology [2]. One of the most successful models was proposed by Kuramoto [3], who assumed that each member of the population can be modeled as an oscillator moving in a globally attracting limit cycle of constant amplitude. The interactions between the oscillators are sufficiently weak to ensure that perturbations will not move them away from their individual limit cycles. Only one degree of freedom, the phase $\theta_{i}$, is then required to describe the dynamics of each oscillator $i$. The latter evolves according to $\frac{d}{d t} \theta_{i}=\omega_{i}+\sum_{j} K_{i j} f\left(\theta_{i}-\theta_{j}\right)$. Here $\omega_{i}$ is the natural frequency of oscillator $i$ (drawn randomly from some distribution), $K_{i j}$ is the coupling strength between oscillators $i$ and $j$, and $f(x)$ a non-linear function. Kuramoto initially considered mean field coupling strengths $K_{i j}=K / N$, where $N$ is the total number of oscillators, and $f(x)=\sin (x)$. This particular model has been investigated intensively. Generally, for some critical (positive) value of the coupling $K$ a phase transition occurs from a state where all the oscillators run incoherently to a state where a certain degree of 
synchronization emerges spontaneously. The details of this transition depend on the the distribution of natural frequencies [4, 5, 6, 7] and the nature of the interactions (viz. short range interactions [8, 9], random site disorder 10], or even more complex types [1]). Kuramoto-type models have in addition been studied extensively in the presence of noise 四, 12, 13.

The equations of the Kuramoto model have also been derived in the context of neural networks [14, 15] and Josephson junctions [16, 17, 18]. There, however, one usually obtains an extra term $A_{i j}$ in the argument of the nonlinear function:

$$
\frac{d}{d t} \theta_{i}=\omega_{i}+\sum_{j} K_{i j} f\left(\theta_{i}-\theta_{j}+A_{i j}\right)
$$

The simplest case, $A_{i j}=\alpha$ for all $(i, j)$, was studied in [19]. Here the degree of spontaneous synchronization was found to be maximal when $\alpha=0$, decreasing monotonically to zero as $\alpha \rightarrow \frac{\pi}{2}$. A similar, but more complicated case is the subject of [20]. However, the problem is far from solved for the case of quenched bond disorder. Here only simulation studies [21, 22] have been published so far; these emphasize the crucial role played by the disorder in determining the long time properties of the model, as measured by correlation functions. The situation is even more complex when random pinning fields are added, leading to further competition.

The simplest models with randomness in both interactions and external fields are those where all oscillators have the same natural frequency, $\omega_{i}=\omega$; here one can switch to a new basis and transform away the $\omega_{i}$. Moreover, if the matrix $\left\{A_{i j}\right\}$ fulfills certain symmetry criteria, then (1) can be written as a gradient descent process, enabling (upon adding Gaussian white noise) an equilibrium statistical mechanical analysis. Low dimensional versions of the resulting system have also been studied; they are equivalent to frustrated XY models, used to describe Josephson junction arrays. Here the sum over interacting pairs in the Hamiltonian is restricted to neighbours which form a plaquette, with $\theta_{i}$ denoting the orientation of the spin at site $i$, and $A_{i j}$ denoting the bond angle, such that the plaquette sum $\sum_{(i, j) \in \text { plaq }} A_{i j}=2 \pi f$, where $f$ is a measure of the frustration. For $f=1 / 2$ one has the so-called fully frustrated model. Both the critical behaviour and the symmetries of these low dimensional systems are known to depend highly discontinuously on $f$ [23. Random external fields trigger local fluctuations of $f$, giving rise to a complex phenomenology which is still subject of study. It would be interesting to investigate whether such features remain in meanfield models. To our knowledge, this has not yet been studied; the effect of random fields has so far only been analyzed in systems without quenched disorder [24, or for $m$-vector spin glass models 22, 26, 27] (but for local uni-axial an-isotropic fields of a different nature than the ones discussed here).

Our paper is structured as follows. We first define two mean-field models with disorder in both bonds and pinning fields, and their appropriate macroscopic observables. The first model is of a conventional type, with individual pairs of oscillators trying to either fully synchronize or fully anti-synchronize, depending on the sign of their interaction. The second model is less conventional in that neuron pairs prefer phase differences $A_{i j}$ of either $\pi / 2$ or $-\pi / 2$. For both models the presence of random external pinning forces increases energetic conflicts and frustration further. In section three we solve both models in equilibrium, using the replica method; we make the replica-symmetric ansatz and calculate the conditions for replicon instabilities. In sections four and five we study the effects of global symmetries. Finally, in section six we present results (in the form of phase diagrams and the temperature and field 
strength dependence of observables) for a number of specific choices for the pinning field distributions, and validate our predictions via numerical simulations.

\section{Model definitions}

We study systems of $N$ coupled Kuramoto oscillators (or XY spins) with external pinning fields, described by Hamiltonians of the form

$$
H=-\frac{2 K}{\sqrt{N}} \sum_{i<j} \cos \left(\theta_{i}-\theta_{j}+A_{i j}\right)-h \sum_{i} \cos \left(\theta_{i}-\phi_{i}\right)
$$

where $\theta_{i} \in[0,2 \pi]$ denotes the phase of the $i$-th oscillator. The natural dynamics leading to a Boltzmann state with Hamiltonian (2) is the Langevin equation $\frac{d}{d t} \theta_{i}=$ $-\partial H / \partial \theta_{i}+\xi_{i}(t)$, with Gaussian random forces $\xi_{i}(t)$ which obey $\left\langle\xi_{i}(t)\right\rangle=0$ and $\left\langle\xi_{i}(t) \xi_{j}\left(t^{\prime}\right)\right\rangle=2 T \delta_{i j} \delta\left[t-t^{\prime}\right]$. Working out this expression gives

$$
\begin{aligned}
\frac{d}{d t} \theta_{i}= & -\frac{2 K}{\sqrt{N}} \sum_{k \neq i}\left[\cos \left(\tilde{A}_{i k}\right) \sin \left(\theta_{i}-\theta_{k}\right)+\sin \left(\tilde{A}_{i k}\right) \cos \left(\theta_{i}-\theta_{k}\right)\right] \\
& -h \sin \left(\theta_{i}-\phi_{i}\right)+\xi_{i}(t)
\end{aligned}
$$

with $\tilde{A}_{i k}=A_{i k}$ for $i<j$, and $\tilde{A}_{i k}=-A_{k i}$ for $i>j$. We introduce disorder by drawing the pinning angles $\phi_{i}$ independently at random from a distribution $p(\phi)$, and the relative angles $A_{i j}$ independently at random from a distribution $P(A)$, with $\int d A P(A) \cos (A)=0$ (to ensure that the bond disorder will retain significance for $N \rightarrow \infty)$. This system will generally exhibit competition between alignment of the spins to the pinning fields and the realization of prescribed relative angles between pairs, and have a high degree of frustration. Each pair $(i, j)$ of spins try to achieve $\theta_{i}-\theta_{j}+\tilde{A}_{i j}=0$, so a bond-frustrated loop $\left\{i_{1}, \ldots, i_{L}\right\}$ is one where $\tilde{A}_{i_{1} i_{2}}+\tilde{A}_{i_{2} i_{3}}+\ldots+\tilde{A}_{i_{L-1} i_{L}}+\tilde{A}_{i_{L} i_{1}} \neq 0(\bmod 2 \pi)$. We simplify the bond average by choosing the $\left\{A_{i j}\right\}$ to be binary: $P(A)=\frac{1}{2} \delta\left[A-A^{\star}\right]+\frac{1}{2} \delta\left[A-A^{\star}-\pi\right]$. Without loss of generality we may take $-\frac{\pi}{2}<A^{\star} \leq \frac{\pi}{2}$. However, only for $A^{\star} \in\left\{0, \frac{1}{2} \pi\right\}$ will it be possible to calculate the disorder-averaged free energy for the system (2) in terms of a standard replica mean field theory; these two cases are the subject of our paper.

Our first (conventional) model corresponds to $P\left(A_{i j}\right)=\frac{1}{2} \delta\left[A_{i j}\right]+\frac{1}{2} \delta\left[A_{i j}-\pi\right]$ :

$$
\text { Model I : } \quad H=-\frac{2 K}{\sqrt{N}} \sum_{i<j} J_{i j} \cos \left(\theta_{i}-\theta_{j}\right)-h \sum_{i} \cos \left(\theta_{i}-\phi_{i}\right)
$$

with $J_{i j}=\cos \left(A_{i j}\right) \in\{-1,1\}$. In this model energy minimization will translate for any pair $(i, j)$ of oscillators into the objectives

$$
\begin{aligned}
& J_{i j}=1: \quad \theta_{i}-\theta_{j} \rightarrow 0 \quad(\bmod 2 \pi) \\
& J_{i j}=-1: \quad \theta_{i}-\theta_{j} \rightarrow \pi \quad(\bmod 2 \pi)
\end{aligned}
$$

(i.e. ferro- and anti-ferromagnetic interactions, respectively). The Hamiltonian (4) defines a Gibbs measure corresponding to the Langevin forces $f_{i}=-\partial H / \partial \theta_{i}=$ $-(2 K / \sqrt{N}) \sum_{k \neq i} J_{i k} \sin \left(\theta_{i}-\theta_{k}\right)-h \sin \left(\theta_{i}-\phi_{i}\right)$, provided we define $J_{i j}=J_{j i}$. A triplet of spins $(i, j, k)$ is now bond-frustrated if an odd number of the relative angles involved equals -1 , and unfrustrated otherwise (see figure 1). Our second (less conventional) model is obtained for $P\left(A_{i j}\right)=\frac{1}{2} \delta\left[A_{i j}-\frac{\pi}{2}\right]+\frac{1}{2} \delta\left[A_{i j}+\frac{\pi}{2}\right]$ :

$$
\text { Model II : } \quad H=-\frac{2 K}{\sqrt{N}} \sum_{i<j} J_{i j} \sin \left(\theta_{i}-\theta_{j}\right)-h \sum_{i} \cos \left(\theta_{i}-\phi_{i}\right)
$$


Model I:

$A_{i j} \in\{0, \pi\}$

Model II:

$A_{i j} \in\left\{-\frac{\pi}{2}, \frac{\pi}{2}\right\}$
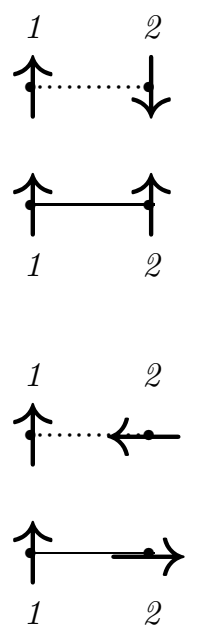
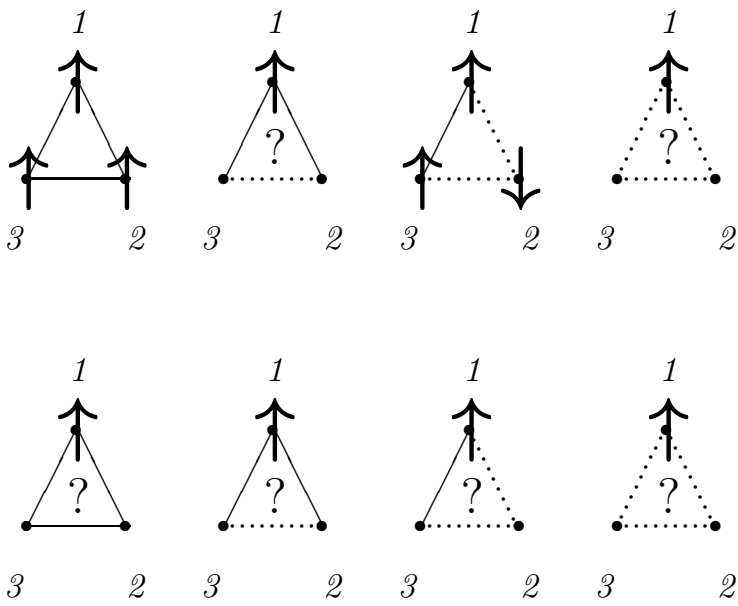

Figure 1. The low energy states of the two models (4) (upper row) and (5) (lower row), for $N \in\{2,3\}$ and in the absence of pinning fields. Arrows indicate the orientations of the vectors $\mathbf{S}_{i}=\left(\cos \left(\theta_{i}\right), \sin \left(\theta_{i}\right)\right)$. Without pinning fields only relative angles are important, so we may put $\theta_{1}=\frac{1}{2} \pi$. The bonds $J_{i j} \in\{-1,1\}$ are drawn as solid (1) and dotted (-1) line segments, respectively, which connect the vectors $\mathbf{S}_{i}$ and $\mathbf{S}_{j}$. Frustrated clusters, where no state exists which minimizes all terms in the Hamiltonian simultaneously, are indicated with '?'. In model I the triplet $(1,2,3)$ is bond-frustrated if an odd number of the bonds $\left\{J_{12}, J_{13}, J_{23}\right\}$ equals -1 . In model II the triplet $(1,2,3)$ is always bond-frustrated.

with $J_{i j}=-\sin \left(A_{i j}\right) \in\{-1,1\}$. Here energy minimization will translate for any pair $(i, j)$ of oscillators into the objectives

$$
\begin{aligned}
& J_{i j}=1: \quad \theta_{i}-\theta_{j} \rightarrow \pi / 2 \quad(\bmod 2 \pi) \\
& J_{i j}=-1: \quad \theta_{i}-\theta_{j} \rightarrow-\pi / 2 \quad(\bmod 2 \pi)
\end{aligned}
$$

The Hamiltonian (4) defines a Gibbs measure corresponding to the Langevin forces $f_{i}=-\partial H / \partial \theta_{i}=-(2 K / \sqrt{N}) \sum_{k \neq i} J_{i j} \cos \left(\theta_{i}-\theta_{k}\right)-h \sin \left(\theta_{i}-\phi_{i}\right)$, provided we define $J_{i j}=-J_{j i}$. Now every triplet $(i, j, k)$ is bond-frustrated, since with $A_{i j} \in\left\{-\frac{\pi}{2}, \frac{\pi}{2}\right\}$ one always has $\tilde{A}_{i j}+\tilde{A}_{j k}+\tilde{A}_{i k}=\ell \pi / 2$, with $\ell$ integer and odd (see figure 1).

It is not a priori clear whether and how the solutions of models (4) and (5) are related. For instance, finding their ground states for $N=3$ reduces to minimizing $H_{\mathrm{I}}\left(\theta_{1}, \theta_{2}\right)=J_{1} \cos \left(\theta_{1}\right)+J_{2} \cos \left(\theta_{2}\right)+J_{3} \cos \left(\theta_{1}-\theta_{2}\right)$ and $H_{\mathrm{II}}\left(\theta_{1}, \theta_{2}\right)=J_{1} \sin \left(\theta_{1}\right)+$ $J_{2} \sin \left(\theta_{2}\right)+J_{3} \sin \left(\theta_{1}-\theta_{2}\right)$, respectively (since rotation invariance allows us to put $\theta_{3}=0$ ), with $J_{i} \in\{-1,1\}$ (chosen randomly). Solving this simple problem reveals that the ground state energy $E_{\mathrm{I}}$ of $H_{\mathrm{I}}$ is dependent on the bonds $\left\{J_{i}\right\}, E_{\mathrm{I}} \in\{-1,-3\}$. Averaging over the bond realizations gives $\overline{E_{\mathrm{I}}}=-2$. In contrast, for the ground state energy of model II one finds $E_{\mathrm{II}}=-\frac{3}{2} \sqrt{3}$, independent of the bond realization.

Pinning fields break the global rotation invariance of (2) which is present for $h=0$, and simplifications of our equations will thus have to be based on symmetries of the pinning field distribution. Any non-zero value of the average pinning direction $\langle\phi\rangle_{\phi}$ can be transformed away, via $\theta_{i} \rightarrow \theta_{i}+\langle\phi\rangle_{\phi}$, without affecting the interaction terms in our Hamiltonian. Thus, without loss of generality we may choose $\langle\phi\rangle_{\phi}=0$. In the remainder of this paper we restrict ourselves, for simplicity, to reflection-symmetric 
pinning field distributions, where $p(\phi)=p(-\phi)$ for all $\phi \in[-\pi, \pi]$. However, the free energies of our models (4,5) are both invariant

$$
p(\phi) \rightarrow \frac{1}{2}(1+\eta) p\left(\phi-\lambda-\frac{\pi}{2}\right)+\frac{1}{2}(1-\eta) p\left(\phi-\lambda+\frac{\pi}{2}\right)
$$

for any $\eta \in[-1,1]$ and $\lambda \in[0,2 \pi]$, so we solve implicitly for a larger family of models. For high temperatures we expect the symmetries of $p(\phi)$ to be inherited by the solutions of our models. We define a complex (single-site) disorder-averaged susceptibility $\chi$, upon adding small perturbations to the external pinning angles $\phi_{i}$ :

$$
\chi=\frac{1}{i N h} \sum_{i} \overline{\left[e^{-i \phi_{i}} \frac{\partial}{\partial \phi_{i}}\left\langle e^{i \theta_{i}}\right\rangle\right]}
$$

Here $\langle\ldots\rangle$ and $\overline{[\ldots]}$ denote equilibrium and disorder averages, respectively. For perfectly linear single-site response, i.e. $\left\langle e^{i \theta_{i}}\right\rangle=x e^{i \phi_{i}}$, definition (7) would give $\chi=x / h$. Evaluating (7) for systems with Hamiltonians of the form (2) gives

$$
\chi=\frac{\beta}{i N} \sum_{i} \overline{\left[\left\langle\sin \left(\theta_{i}-\phi_{i}\right) e^{i\left(\theta_{i}-\phi_{i}\right)}\right\rangle-\left\langle\sin \left(\theta_{i}-\phi_{i}\right)\right\rangle\left\langle e^{i\left(\theta_{i}-\phi_{i}\right)}\right\rangle\right]}
$$

\section{Equilibrium analysis}

\subsection{Solution via replica theory}

We calculate the disorder-averaged free energy per spin $\bar{f}=-\lim _{N \rightarrow \infty}(\beta N)^{-1} \overline{\log Z}$ for the Hamiltonian (2), using the identity $\overline{\log Z}=\lim _{n \rightarrow 0} n^{-1} \log \overline{Z^{n}}$ and the standard manipulations of conventional replica theory [29], giving

$$
\bar{f}=-\lim _{N \rightarrow \infty} \lim _{n \rightarrow 0} \frac{1}{\beta N n} \log \int \ldots \int\left[\prod_{\alpha=1}^{n} d \boldsymbol{\theta}^{\alpha}\right] \overline{e^{-\beta \sum_{\alpha} H\left(\boldsymbol{\theta}^{\alpha}\right)}}
$$

The disorder average, with $P(A)=\frac{1}{2} \delta\left[A-A^{\star}\right]+\frac{1}{2} \delta\left[A-A^{\star}-\pi\right]$, gives:

$$
\overline{e^{-\beta \sum_{\alpha} H\left(\boldsymbol{\theta}^{\alpha}\right)}}=e^{\beta h \sum_{i \alpha} \cos \left(\theta_{i}^{\alpha}-\phi_{i}\right)} \prod_{i<j} \cosh \left[\frac{2 \beta K}{\sqrt{N}} \sum_{\alpha} \cos \left(\theta_{i}^{\alpha}-\theta_{j}^{\alpha}+A^{\star}\right)\right]
$$

Expansion for large $N$, however, only leads to an expression in terms of the usual single-site replica order parameters if $\sin \left(2 A^{\star}\right)=0$, i.e. for $A^{\star} \in\left\{0, \frac{\pi}{2}\right\}$ (因河). Here

$$
\begin{aligned}
\overline{e^{-\beta \sum_{\alpha} H\left(\boldsymbol{\theta}^{\alpha}\right)}} & =e^{\beta h \sum_{i \alpha} \cos \left(\theta_{i}^{\alpha}-\phi_{i}\right)+2 \beta^{2} K^{2} N \sin ^{2}\left(A^{\star}\right) \sum_{\alpha \beta}\left[q_{\alpha \beta}^{s s}(\boldsymbol{\theta}) q_{\alpha \beta}^{c c}(\boldsymbol{\theta})-q_{\alpha \beta}^{s c}(\boldsymbol{\theta}) q_{\alpha \beta}^{c s}(\boldsymbol{\theta})\right]+\mathcal{O}\left(N^{0}\right)} \\
& \times e^{\beta^{2} K^{2} N \cos ^{2}\left(A^{\star}\right) \sum_{\alpha \beta}\left[q_{\alpha \beta}^{c c}(\boldsymbol{\theta}) q_{\alpha \beta}^{c c}(\boldsymbol{\theta})+q_{\alpha \beta}^{s s}(\boldsymbol{\theta}) q_{\alpha \beta}^{s s}(\boldsymbol{\theta})+q_{\alpha \beta}^{c s}(\boldsymbol{\theta}) q_{\alpha \beta}^{c s}(\boldsymbol{\theta})+q_{\alpha \beta}^{s c}(\boldsymbol{\theta}) q_{\alpha \beta}^{s c}(\boldsymbol{\theta})\right]}
\end{aligned}
$$

with

$$
\begin{aligned}
q_{\alpha \beta}^{c c}(\boldsymbol{\theta}) & =\frac{1}{N} \sum_{i} \cos \left(\theta_{i}^{\alpha}\right) \cos \left(\theta_{i}^{\beta}\right), & q_{\alpha \beta}^{s s}(\boldsymbol{\theta}) & =\frac{1}{N} \sum_{i} \sin \left(\theta_{i}^{\alpha}\right) \sin \left(\theta_{i}^{\beta}\right) \\
q_{\alpha \beta}^{s c}(\boldsymbol{\theta}) & =\frac{1}{N} \sum_{i} \sin \left(\theta_{i}^{\alpha}\right) \cos \left(\theta_{i}^{\beta}\right), & q_{\alpha \beta}^{c s}(\boldsymbol{\theta}) & =\frac{1}{N} \sum_{i} \cos \left(\theta_{i}^{\alpha}\right) \sin \left(\theta_{i}^{\beta}\right)
\end{aligned}
$$

$\ddagger$ The transformation (6) implies $\phi_{i} \rightarrow \phi_{i}+\lambda+\tau_{i} \frac{\pi}{2}$, where $\tau_{i} \in\{-1,1\}$. The variables $\lambda$ and $\left\{\tau_{i}\right\}$ can be gauged away by putting $\theta_{i} \rightarrow \theta_{i}+\lambda+\tau_{i} \frac{\pi}{2}$ in combination with $J_{i j} \rightarrow J_{i j} \cos \left(\frac{\pi}{2}\left(\tau_{i}-\tau_{j}\right)\right)$. 
We proceed as usual, isolating observables via appropriate $\delta$-distributions (which introduces conjugate order parameters), leading to site factorisation. The result is

$$
\begin{aligned}
& \bar{f}=-\lim _{n \rightarrow 0} \frac{1}{n} \operatorname{extr}\left\{\Phi\left[\left\{\hat{\boldsymbol{q}}^{\star \star}, \boldsymbol{q}^{\star \star}\right\}\right]+\Psi\left[\left\{\hat{\boldsymbol{q}}^{\star \star}\right\}\right]\right\} \\
& \Phi[\ldots]=\frac{i}{\beta} \sum_{\star \star} \sum_{\alpha \beta} \hat{q}_{\alpha \beta}^{\star \star} q_{\alpha \beta}^{\star \star}+\beta K^{2} \cos ^{2}\left(A^{\star}\right) \sum_{\star \star} \sum_{\alpha \beta}\left[q_{\alpha \beta}^{\star \star}\right]^{2} \\
&+2 \beta K^{2} \sin ^{2}\left(A^{\star}\right) \sum_{\alpha \beta}\left[q_{\alpha \beta}^{s s} q_{\alpha \beta}^{c c}-q_{\alpha \beta}^{s c} q_{\alpha \beta}^{c s}\right] \\
& \beta \Psi[\ldots]=\left\langle\log \int d \boldsymbol{\theta} M\left(\boldsymbol{\theta} \mid\left\{\hat{\boldsymbol{q}}^{\star \star}\right\}\right)\right\rangle_{\phi}
\end{aligned}
$$

with $(\star \star) \in\{(c c),(s s),(s c),(c s)\}$, and with

$$
\begin{aligned}
M\left(\boldsymbol{\theta} \mid\left\{\hat{\boldsymbol{q}}^{\star \star}\right\}\right)= & e^{\beta h \sum_{\alpha} \cos \left(\theta^{\alpha}-\phi\right)-i \sum_{\alpha \beta}\left[\hat{q}_{\alpha \beta}^{c c} \cos \left(\theta_{\alpha}\right) \cos \left(\theta_{\beta}\right)+\hat{q}_{\alpha \beta}^{c s} \cos \left(\theta_{\alpha}\right) \sin \left(\theta_{\beta}\right)\right]} \\
& \times e^{-i \sum_{\alpha \beta}\left[\hat{q}_{\alpha \beta}^{s s} \sin \left(\theta_{\alpha}\right) \sin \left(\theta_{\beta}\right)+\hat{q}_{\alpha \beta}^{s c} \sin \left(\theta_{\alpha}\right) \cos \left(\theta_{\beta}\right)\right]}
\end{aligned}
$$

One always has $q_{\lambda \rho}^{c s}=q_{\rho \lambda}^{s c}$, which allows us to simplify the saddle point equations. For both models we find upon varying the conjugate order parameters $\left\{\hat{q}_{\alpha \beta}^{\star \star}\right\}$ :

$$
\begin{aligned}
& q_{\lambda \rho}^{c c}=\left\langle\frac{\int d \boldsymbol{\theta} \cos \left(\theta_{\lambda}\right) \cos \left(\theta_{\rho}\right) M\left(\boldsymbol{\theta} \mid\left\{\hat{\boldsymbol{q}}^{\star \star}\right\}\right)}{\int d \boldsymbol{\theta} M\left(\boldsymbol{\theta} \mid\left\{\hat{\boldsymbol{q}}^{\star \star}\right\}\right)}\right\rangle_{\phi} \\
& q_{\lambda \rho}^{s s}=\left\langle\frac{\int d \boldsymbol{\theta} \sin \left(\theta_{\lambda}\right) \sin \left(\theta_{\rho}\right) M\left(\boldsymbol{\theta} \mid\left\{\hat{\boldsymbol{q}}^{\star \star}\right\}\right)}{\int d \boldsymbol{\theta} M\left(\boldsymbol{\theta} \mid\left\{\hat{\boldsymbol{q}}^{\star \star}\right\}\right)}\right\rangle_{\phi} \\
& q_{\lambda \rho}^{s c}=\left\langle\frac{\int d \boldsymbol{\theta} \sin \left(\theta_{\lambda}\right) \cos \left(\theta_{\rho}\right) M\left(\boldsymbol{\theta} \mid\left\{\hat{\boldsymbol{q}}^{\star \star}\right\}\right)}{\int d \boldsymbol{\theta} M\left(\boldsymbol{\theta} \mid\left\{\hat{\boldsymbol{q}}^{\star \star}\right\}\right)}\right\rangle_{\phi}
\end{aligned}
$$

The models I $\left(A^{\star}=0\right)$ and II $\left(A^{\star}=\frac{\pi}{2}\right)$ differ only in the equations resulting from variation of the order parameters $\left\{q_{\alpha \beta}^{\star \star}\right\}$ :

$$
\begin{array}{lll}
\text { Model I : } & \hat{q}_{\alpha \beta}^{c c}=2 i \beta^{2} K^{2} q_{\alpha \beta}^{c c}, & \hat{q}_{\alpha \beta}^{s s}=2 i \beta^{2} K^{2} q_{\alpha \beta}^{s s} \\
& \hat{q}_{\alpha \beta}^{c s}=2 i \beta^{2} K^{2} q_{\alpha \beta}^{c s}, & \hat{q}_{\alpha \beta}^{s c}=2 i \beta^{2} K^{2} q_{\alpha \beta}^{s c} \\
\text { Model II : } & \hat{q}_{\alpha \beta}^{c c}=2 i \beta^{2} K^{2} q_{\alpha \beta}^{s s}, & \hat{q}_{\alpha \beta}^{s s}=2 i \beta^{2} K^{2} q_{\alpha \beta}^{c c} \\
& \hat{q}_{\alpha \beta}^{c s}=-2 i \beta^{2} K^{2} q_{\alpha \beta}^{s c}, & \hat{q}_{\alpha \beta}^{s c}=-2 i \beta^{2} K^{2} q_{\alpha \beta}^{c s}
\end{array}
$$

\subsection{Replica-symmetric solutions}

We now make the replica-symmetric (or ergodic) ansatz (RS) for the saddle-points:

$$
\begin{aligned}
& q_{\alpha \beta}^{\star \star}=\left(Q_{\star \star}-q_{\star \star}\right) \delta_{\alpha \beta}+q_{\star \star} \\
& \hat{q}_{\alpha \beta}^{\star \star}=\frac{1}{2} i(2 \beta K)^{2}\left(R_{\star \star}-r_{\star \star}\right) \delta_{\alpha \beta}+\frac{1}{2} i(2 \beta K)^{2} r_{\star \star}
\end{aligned}
$$

Insertion into the saddle-point equations tells us that

$$
\begin{array}{ll}
R_{c c}=\cos ^{2}\left(A^{\star}\right) Q_{c c}+\sin ^{2}\left(A^{\star}\right) Q_{s s} & r_{c c}=\cos ^{2}\left(A^{\star}\right) q_{c c}+\sin ^{2}\left(A^{\star}\right) q_{s s} \\
R_{s s}=\cos ^{2}\left(A^{\star}\right) Q_{s s}+\sin ^{2}\left(A^{\star}\right) Q_{c c} & r_{s s}=\cos ^{2}\left(A^{\star}\right) q_{s s}+\sin ^{2}\left(A^{\star}\right) q_{c c} \\
R_{c s}=\cos \left(2 A^{\star}\right) Q_{c s} & r_{c s}=\cos \left(2 A^{\star}\right) q_{c s}
\end{array}
$$


The identity $Q_{c c}+Q_{s s}=1$ allows us to reduce the problem to solving five coupled non-linear equations. We will compactify our notation by using the short-hands

$$
\left\langle\langle\ldots\rangle=\int D x D y D u D v \ldots \quad\langle f(\theta)\rangle_{\star}=\frac{\int d \theta f(\theta) M(\theta \mid x, y, u, v, \phi)}{\int d \theta M(\theta \mid x, y, u, v, \phi)}\right.
$$

We can write the five remaining saddle-point equations as

$$
\begin{aligned}
& Q_{c c}=\left\langle\left\langle\left\langle\left\langle\cos ^{2}(\theta)\right\rangle_{\star}\right\rangle\right\rangle_{\phi} \quad Q_{c s}=\left\langle\left\langle\left\langle\langle\cos (\theta) \sin (\theta)\rangle_{\star}\right\rangle\right\rangle\right\rangle_{\phi}\right. \\
& q_{c c}=\left\langle\left\langle\left\langle\langle\cos (\theta)\rangle_{\star}^{2}\right\rangle\right\rangle\right\rangle_{\phi} \quad q_{s s}=\left\langle\left\langle\left\langle\langle\sin (\theta)\rangle_{\star}^{2}\right\rangle\right\rangle_{\phi}\right. \\
& q_{c s}=\left\langle\left\langle\left\langle\langle\cos (\theta)\rangle_{\star}\langle\sin (\theta)\rangle_{\star}\right\rangle\right\rangle\right\rangle_{\phi}
\end{aligned}
$$

Tracing back the physical meaning of these observables gives:

$$
\begin{aligned}
Q_{c c} & =\lim _{N \rightarrow \infty} \frac{1}{N} \sum_{i} \overline{\left\langle\cos ^{2}\left(\theta_{i}\right)\right\rangle}, \quad Q_{c s}=\lim _{N \rightarrow \infty} \frac{1}{N} \sum_{i} \overline{\left\langle\cos \left(\theta_{i}\right) \sin \left(\theta_{i}\right)\right\rangle} \\
q_{c c} & =\lim _{N \rightarrow \infty} \frac{1}{N} \sum_{i} \overline{\left\langle\cos \left(\theta_{i}\right)\right\rangle^{2}}, \quad q_{s s}=\lim _{N \rightarrow \infty} \frac{1}{N} \sum_{i} \overline{\left\langle\sin \left(\theta_{i}\right)\right\rangle^{2}} \\
q_{c s} & =\lim _{N \rightarrow \infty} \frac{1}{N} \sum_{i} \overline{\left\langle\cos \left(\theta_{i}\right)\right\rangle\left\langle\sin \left(\theta_{i}\right)\right\rangle}
\end{aligned}
$$

Our two models differ only in the form taken by the effective single spin measure $M(\theta \mid x, y, u, v, \phi)$ in the RS saddle-point equations:

$$
\begin{aligned}
& M_{\mathrm{I}}(\theta \mid x, y, u, v, \phi)=e^{\beta h \cos (\theta-\phi)+(\beta K)^{2}\left[\left[2 Q_{c c}-1+q_{s s}-q_{c c}\right] \cos (2 \theta)+2\left(Q_{c s}-q_{c s}\right) \sin (2 \theta)\right]} \\
& \quad \times e^{\beta K \cos (\theta) \sqrt{2}\left[x \sqrt{2} \sqrt{q_{c c}}+(u-i v) \sqrt{q_{c s}}\right]+\beta K \sin (\theta) \sqrt{2}\left[y \sqrt{2} \sqrt{q_{s s}}+(u+i v) \sqrt{q_{c s}}\right]} \\
& \quad \times M_{\mathrm{II}}(\theta \mid x, y, u, v, \phi)=e^{\beta h \cos (\theta-\phi)-(\beta K)^{2}\left[\left[2 Q_{c c}-1+q_{s s}-q_{c c}\right] \cos (2 \theta)+2\left(Q_{c s}-q_{c s}\right) \sin (2 \theta)\right]} \\
& \quad \times e^{\beta K \cos (\theta) \sqrt{2}\left[x \sqrt{2} \sqrt{q_{s s}}+(i u+v) \sqrt{q_{c s}}\right]+\beta K \sin (\theta) \sqrt{2}\left[y \sqrt{2} \sqrt{q_{c c}}+(i u-v) \sqrt{q_{c s}}\right]}
\end{aligned}
$$

(apart, in both cases, from a constant pre-factor $e^{(\beta K)^{2}\left[1-q_{c c}-q_{s s}\right]}$ ). To compactify future notation we define the following short-hands

$$
\begin{aligned}
& \gamma_{c c}=\left\langle\cos ^{2}(\theta)\right\rangle_{\star}-\langle\cos (\theta)\rangle_{\star}^{2} \quad \gamma_{s s}=\left\langle\sin ^{2}(\theta)\right\rangle_{\star}-\langle\sin (\theta)\rangle_{\star}^{2} \\
& \gamma_{c s}=\langle\cos (\theta) \sin (\theta)\rangle_{\star}-\langle\cos (\theta)\rangle_{\star}\langle\sin (\theta)\rangle_{\star}
\end{aligned}
$$

Note that in replica-symmetric states one has $\left\langle\left\langle\left\langle\gamma_{c c}\right\rangle\right\rangle_{\phi}=Q_{c c}-q_{c c},\left\langle\left\langle\left\langle\gamma_{s s}\right\rangle\right\rangle_{\phi}=\right.\right.$ $1-Q_{c c}-q_{s s},\left\langle\left\langle\left\langle\gamma_{c s}\right\rangle\right\rangle\right\rangle_{\phi}=Q_{c s}-q_{c s}$, and $\left|\gamma_{c s}\right| \leq \frac{1}{2}\left(\gamma_{c c}+\gamma_{s s}\right)$.

Similarly we can work out the RS free energy per oscillator, by insertion of the RS ansatz into (9), followed by standard manipulations. This results in

$$
\begin{aligned}
& \bar{f}=\operatorname{extr}_{\left\{Q_{\star \star}, q_{\star \star}\right\}} f\left[\left\{Q_{\star \star}, q_{\star \star}\right\}\right] \\
& f[\ldots]=\beta K^{2} U-\frac{1}{\beta} \int D x D y D u D v\left\langle\log \int d \theta M(\theta \mid x, y, u, v, \phi)\right\rangle_{\phi}
\end{aligned}
$$

with the effective measures $M(\theta \mid \ldots)$ defined in (26) 27), and with

$$
\begin{aligned}
& U_{\mathrm{I}}=2 Q_{c c}\left(Q_{c c}-1\right)+2 Q_{c s}^{2}-q_{c c}\left(q_{c c}-1\right)-q_{s s}\left(q_{s s}-1\right)-2 q_{c s}^{2} \\
& U_{\mathrm{II}}=-2 Q_{c c}\left(Q_{c c}-1\right)-1-2 Q_{c s}^{2}+q_{c c}+q_{s s}-2 q_{c c} q_{s s}+2 q_{c s}^{2}
\end{aligned}
$$


The nature of the extremum in (30) follows from the high-temperature state. Expanding for $\beta \rightarrow 0$ gives $f[\ldots]=-\frac{1}{\beta} \log (2 \pi)-\frac{1}{4} \beta h^{2}+2 \beta K^{2} \operatorname{extr}\{\tilde{U}\}+\mathcal{O}\left(\beta^{2}\right)$, with

$$
\begin{aligned}
& \tilde{U}_{\mathrm{I}}=Q_{c c}^{2}-Q_{c c}+Q_{c s}^{2}-\frac{1}{2}\left(q_{c c}^{2}+q_{s s}^{2}\right)-q_{c s}^{2} \\
& \tilde{U}_{\mathrm{II}}=-Q_{c c}^{2}+Q_{c c}-\frac{1}{2}-Q_{c s}^{2}-q_{c c} q_{s s}+q_{c s}^{2}
\end{aligned}
$$

For $\beta \rightarrow 0$ we must find the paramagnetic solution $Q_{c c}=\frac{1}{2}$ and $Q_{c s}=q_{c c}=q_{s s}=$ $q_{c s}=0$, so the nature of the desired extremum in (30) is

$$
\begin{array}{lll}
\text { I : } & \min \text { w.r.t. }\left\{Q_{c c}, Q_{c s}\right\}, & \max \text { w.r.t. }\left\{q_{c c}, q_{s s}, q_{c s}\right\} \\
\text { II : } & \min \text { w.r.t. }\left\{q_{c s}, q_{c c}-q_{s s}\right\}, & \max \text { w.r.t. }\left\{Q_{c c}, Q_{c s}, q_{c c}+q_{s s}\right\}
\end{array}
$$

Finally, for RS solutions we can also work out expression (8) for $\chi$ in the limit $N \rightarrow \infty$ :

$$
\begin{aligned}
\frac{\chi_{\mathrm{RS}}}{\beta} & =\left\langle\left\langle\left\langle\left\langle\sin ^{2}(\theta-\phi)\right\rangle_{\star}-\langle\sin (\theta-\phi)\rangle_{\star}^{2}\right\rangle\right\rangle_{\phi}\right. \\
& -i\left\langle\left\langle\left\langle\langle\sin (\theta-\phi) \cos (\theta-\phi)\rangle_{\star}-\langle\sin (\theta-\phi)\rangle_{\star}\langle\cos (\theta-\phi)\rangle_{\star}\right\rangle\right\rangle\right\rangle_{\phi}
\end{aligned}
$$

Equivalently, with the conventions $(28,29)$ this can be written as

$$
\begin{aligned}
\frac{\chi_{\mathrm{RS}}}{\beta}= & \frac{1}{2}\left(1-q_{c c}-q_{s s}\right)-\left\langle\left\langle\left\langle\frac{1}{2} \cos (2 \phi)\left(\gamma_{c c}-\gamma_{s s}\right)+\sin (2 \phi) \gamma_{c s}\right\rangle\right\rangle\right\rangle_{\phi} \\
& -i\left\langle\left\langle\left\langle\cos (2 \phi) \gamma_{c s}-\frac{1}{2} \sin (2 \phi)\left(\gamma_{c c}-\gamma_{s s}\right)\right\rangle\right\rangle_{\phi}\right.
\end{aligned}
$$

\subsection{The AT lines}

Here we determine the stability of our RS solution against 'replicon' perturbations: $q_{\alpha \beta}^{\star \star} \rightarrow q_{\alpha \beta}^{\star \star, \mathrm{RS}}+\eta_{\alpha \beta}^{\star \star}$ with $\eta_{\alpha \beta}^{c c}=\eta_{\beta \alpha}^{c c}$ and $\eta_{\alpha \beta}^{s s}=\eta_{\beta \alpha}^{s s}$ (for all $\left.\alpha, \beta\right), \eta_{\alpha \alpha}^{\star \star}=0$ for all $\alpha$, $\sum_{\alpha} \eta_{\alpha \beta}^{\star \star}=\sum_{\alpha} \eta_{\beta \alpha}^{\star \star}=0$ for all $\alpha$, and with all $\left|\eta_{\alpha \beta}^{\star \star}\right| \ll 1$. Details of the derivation of the equations signaling the this type of instability (the AT [28] lines), are given in Appendix B. Our calculation is more complicated than that in e.g. [25, 27], because, due to the pinning fields, we cannot generally use rotational symmetry; replicon fluctuations can now have a more complicated structure. We found two types of AT instabilities (the physical RSB transition associated with these is the one occurring at the highest temperature). The first is the same for our two models:

$$
\text { Models I \& II : } \quad(T / 2 K)^{2}=\left\langle\left\langle\left\langle\gamma_{c c} \gamma_{s s}-\gamma_{c s}^{2}\right\rangle\right\rangle\right\rangle_{\phi}
$$

The second AT instability is found to be model dependent:

$$
\begin{array}{ll}
\text { Model I : } & \operatorname{Det}\left[\boldsymbol{E}-(T / 2 K)^{2} \mathbf{I}\right]=0 \\
\text { Model II : } & \operatorname{Det}\left[\boldsymbol{E}-(T / 2 K)^{2} \boldsymbol{C}\right]=0
\end{array}
$$

with

$$
\begin{gathered}
\boldsymbol{C}=\left(\begin{array}{ccc}
1 & 0 & 0 \\
0 & -1 & 0 \\
0 & 0 & -1
\end{array}\right) \\
\boldsymbol{E}=\left(\begin{array}{ccc}
\left\langle\left\langle\left\langle\frac{1}{2}\left(\gamma_{c c}^{2}+\gamma_{s s}^{2}\right)+\gamma_{c s}^{2}\right\rangle\right\rangle\right\rangle_{\phi} & \left\langle\left\langle\left\langle\frac{1}{2}\left(\gamma_{c c}^{2}-\gamma_{s s}^{2}\right)\right\rangle\right\rangle\right\rangle_{\phi} & \left\langle\left\langle\left\langle\gamma_{c s}\left(\gamma_{c c}+\gamma_{s s}\right)\right\rangle\right\rangle_{\phi}\right. \\
\left\langle\left\langle\left\langle\frac{1}{2}\left(\gamma_{c c}^{2}-\gamma_{s s}^{2}\right)\right\rangle\right\rangle_{\phi}\right. & \left\langle\left\langle\left\langle\frac{1}{2}\left(\gamma_{c c}^{2}+\gamma_{s s}^{2}\right)-\gamma_{c s}^{2}\right\rangle\right\rangle\right\rangle_{\phi} & \left\langle\left\langle\left\langle\gamma_{c s}\left(\gamma_{c c}-\gamma_{s s}\right)\right\rangle\right\rangle_{\phi}\right. \\
\left\langle\left\langle\left\langle\gamma_{c s}\left(\gamma_{c c}+\gamma_{s s}\right)\right\rangle\right\rangle\right\rangle_{\phi} & \left\langle\left\langle\left\langle\gamma_{c s}\left(\gamma_{c c}-\gamma_{s s}\right)\right\rangle\right\rangle_{\phi}\right. & \left\langle\left\langle\left\langle\gamma_{c c} \gamma_{s s}+\gamma_{c s}^{2}\right\rangle\right\rangle\right\rangle_{\phi}
\end{array}\right)
\end{gathered}
$$




\section{States with global reflection symmetry}

We inspect the effect of global reflection $\theta_{i} \rightarrow-\theta_{i}$, on the order parameters (within the RS ansatz), with the help of the identifications (23,24,25):

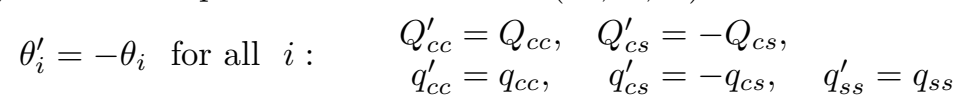

Invariance under this transformation implies $Q_{c s}=q_{c s}=0$, and breaking of the associated symmetry is signaled by a bifurcation of $Q_{c s} \neq 0$ and/or $q_{c s} \neq 0$.

\subsection{Implications for free energy and order parameters}

For reflection-symmetric states the Gaussian variables $\{u, v\}$ disappear from the problem, and the effective single-spin measures (26,27) simplify to

$$
\begin{aligned}
& M_{\mathrm{I}}(\theta \mid x, y, \phi)=e^{\beta h \cos (\theta-\phi)+(\beta K)^{2}\left[2 Q_{c c}-1+q_{s s}-q_{c c}\right] \cos (2 \theta)+2 \beta K\left[\cos (\theta) x \sqrt{q_{c c}}+\sin (\theta) y \sqrt{q_{s s}}\right]} \\
& M_{\mathbb{I}}(\theta \mid x, y, \phi)=e^{\beta h \cos (\theta-\phi)-(\beta K)^{2}\left[2 Q_{c c}-1+q_{s s}-q_{c c}\right] \cos (2 \theta)+2 \beta K\left[\cos (\theta) x \sqrt{q_{s s}}+\sin (\theta) y \sqrt{q_{c c}}\right]}
\end{aligned}
$$

These expressions show, in combination with the underlying symmetry $p(\phi)=p(-\phi)$, that for any set of functions $\left\{k_{\ell}\right\}$ (with $\ell=0,1,2, \ldots$ ):

$$
\left\langle\left\langle\left\langle k_{0}(-\phi) \prod_{\ell>0}\left\langle k_{\ell}(-\theta)\right\rangle_{\star}\right\rangle\right\rangle\right\rangle_{\phi}=\left\langle\left\langle\left\langle k_{0}(\phi) \prod_{\ell>0}\left\langle k_{\ell}(\theta)\right\rangle_{\star}\right\rangle\right\rangle\right\rangle_{\phi}
$$

One is left with just three coupled order parameter equations:

$$
Q_{c c}=\left\langle\left\langle\left\langle\left\langle\cos ^{2}(\theta)\right\rangle_{\star}\right\rangle\right\rangle\right\rangle_{\phi} \quad q_{c c}=\left\langle\left\langle\left\langle\langle\cos (\theta)\rangle_{\star}^{2}\right\rangle\right\rangle\right\rangle_{\phi} \quad q_{s s}=\left\langle\left\langle\left\langle\langle\sin (\theta)\rangle_{\star}^{2}\right\rangle\right\rangle\right\rangle_{\phi}(46)
$$

Inserting $Q_{c s}=q_{c s}=0$ into (30,32, 33) shows that for reflection-symmetric states the free energy per oscillator equals $f=\operatorname{extr}_{\left\{Q_{c c}, q_{s s}, q_{c c}\right\}} f\left[Q_{c c}, q_{s s}, q_{c c}\right]$, with

$$
\begin{array}{r}
f_{\mathrm{I}}\left[Q_{c c}, q_{s s}, q_{c c}\right]=\beta K^{2}\left[q_{c c}+q_{s s}+2 Q_{c c}\left(Q_{c c}-1\right)-q_{c c}^{2}-q_{s s}^{2}\right] \\
-\frac{1}{\beta}\left\langle\int D x D y \log \int d \theta M_{\mathrm{I}}(\theta \mid x, y, \phi)\right\rangle_{\phi} \\
f_{\mathbb{I}}\left[Q_{c c}, q_{s s}, q_{c c}\right]=\beta K^{2}\left[q_{c c}+q_{s s}-2 Q_{c c}\left(Q_{c c}-1\right)-2 q_{s s} q_{c c}-1\right] \\
-\frac{1}{\beta}\left\langle\int D x D y \log \int d \theta M_{\mathbb{I}}(\theta \mid x, y, \phi)\right\rangle_{\phi}
\end{array}
$$

From these expressions one can easily extract the replica symmetric ground states, using a saddle-point argument as $\beta \rightarrow \infty$ in the entropic term:

$$
\begin{aligned}
& \lim _{\beta \rightarrow \infty} f_{\mathrm{I}}[\ldots] / \beta K^{2}=q_{c c}+q_{s s}+2 Q_{c c}\left(Q_{c c}-1\right)-q_{c c}^{2}-q_{s s}^{2}-\left|2 Q_{c c}-1+q_{s s}-q_{c c}\right| \\
& \lim _{\beta \rightarrow \infty} f_{\Pi}[\ldots] / \beta K^{2}=q_{c c}+q_{s s}-2 Q_{c c}\left(Q_{c c}-1\right)-2 q_{s s} q_{c c}-1-\left|2 Q_{c c}-1+q_{s s}-q_{c c}\right|
\end{aligned}
$$

According to (34, 35), we must for model I minimize with respect to $Q_{c c}$ and maximize with respect to $\left\{q_{c c}, q_{s s}\right\}$, whereas for model II we must minimize with respect to $q_{c c}-q_{s s}$ and maximize with respect to $\left\{Q_{c c}, q_{c c}+q_{s s}\right\}$. For both models this leads to

$$
q_{c c}+q_{s s}=1, \quad Q_{c c}=\frac{1}{2}\left[1+q_{c c}-q_{s s}\right]
$$

giving in both cases $\operatorname{extr}_{Q_{c c}, q_{s s}, q_{c c}} \lim _{\beta \rightarrow \infty}\left(\beta K^{2}\right)^{-1} f\left[Q_{c c}, q_{s s}, q_{c c}\right]=0$. Hence there is a finite ground state energy. To find the RS ground states of our two models from the family $q_{c c}+q_{s s}=1$, one would have to inspect the next order in $T$. In addition we must, of course, expect replica symmetry to be broken for low temperatures. 


\subsection{Implications for AT lines and $R S$ susceptibility}

Reflection symmetry is also found to simplify expressions (38,39) for the AT instabilities, due to $\left\langle\left\langle\left\langle\gamma_{c c} \gamma_{c s}\right\rangle\right\rangle\right\rangle_{\phi}=\left\langle\left\langle\left\langle\gamma_{s s} \gamma_{c s}\right\rangle\right\rangle\right\rangle_{\phi}=0$. In particular, with (45) one can simplify the matrix (41) to

$$
\boldsymbol{E}=\left(\begin{array}{ccc}
\left\langle\left\langle\left\langle\frac{1}{2}\left(\gamma_{c c}^{2}+\gamma_{s s}^{2}\right)+\gamma_{c s}^{2}\right\rangle\right\rangle\right\rangle_{\phi} & \left\langle\left\langle\left\langle\frac{1}{2}\left(\gamma_{c c}^{2}-\gamma_{s s}^{2}\right)\right\rangle\right\rangle\right\rangle_{\phi} & 0 \\
\left\langle\left\langle\left\langle\frac{1}{2}\left(\gamma_{c c}^{2}-\gamma_{s s}^{2}\right)\right\rangle\right\rangle\right\rangle_{\phi} & \left\langle\left\langle\left\langle\frac{1}{2}\left(\gamma_{c c}^{2}+\gamma_{s s}^{2}\right)-\gamma_{c s}^{2}\right\rangle\right\rangle\right\rangle_{\phi} & 0 \\
0 & 0 & \left\langle\left\langle\left\langle\gamma_{c c} \gamma_{s s}+\gamma_{c s}^{2}\right\rangle\right\rangle\right\rangle_{\phi}
\end{array}\right)
$$

In combination with (37), and upon rejecting solutions which are immediately seen not to give the highest transition temperature, the AT lines of our two models are found to be the solutions of the following equations, respectively:

I : $\left[\frac{T}{2 K}\right]^{2}=\max \left\{\frac{1}{2}\left\langle\left\langle\left\langle\gamma_{c c}^{2}+\gamma_{s s}^{2}\right\rangle\right\rangle\right\rangle_{\phi}+\sqrt{\left.\left.\left\langle\left\langle\gamma_{c s}^{2}\right\rangle\right\rangle\right\rangle_{\phi}^{2}+\frac{1}{4}\left\langle\left\langle\gamma_{c c}^{2}-\gamma_{s s}^{2}\right\rangle\right\rangle\right\rangle_{\phi}^{2}},\left\langle\left\langle\left\langle\gamma_{c c} \gamma_{s s}+\gamma_{c s}^{2}\right\rangle\right\rangle\right\rangle_{\phi}\right\}$

II : $\left[\frac{T}{2 K}\right]^{2}=\max \left\{\left\langle\left\langle\left\langle\gamma_{c s}^{2}\right\rangle\right\rangle_{\phi}+\sqrt{\left\langle\left\langle\left\langle\gamma_{c c}^{2}\right\rangle\right\rangle_{\phi}\left\langle\left\langle\left\langle\gamma_{s s}^{2}\right\rangle\right\rangle_{\phi}\right.\right.},\left\langle\left\langle\left\langle\gamma_{c c} \gamma_{s s}-\gamma_{c s}^{2}\right\rangle\right\rangle_{\phi}\right\}\right.\right.$

For both models the left of the two arguments in the corresponding extremization problems give the required maximum. To see this, one first notes that

$$
\begin{aligned}
& \left\langle\left\langle\left\langle\gamma_{c c} \gamma_{s s}\right\rangle\right\rangle\right\rangle_{\phi}=\frac{1}{2}\left\langle\left\langle\left\langle\gamma_{c c}^{2}+\gamma_{s s}^{2}-\left(\gamma_{c c}-\gamma_{s s}\right)^{2}\right\rangle\right\rangle\right\rangle_{\phi} \leq \frac{1}{2}\left\langle\left\langle\left\langle\gamma_{c c}^{2}+\gamma_{s s}^{2}\right\rangle\right\rangle\right\rangle_{\phi} \\
& \left\langle\left\langle\left\langle\gamma_{c c}^{2}\right\rangle\right\rangle\right\rangle_{\phi}\left\langle\left\langle\left\langle\gamma_{s s}^{2}\right\rangle\right\rangle_{\phi}=\frac{1}{2}\left\{\left\langle\left\langle\gamma_{c c}^{2}+\gamma_{s s}^{2}\right\rangle\right\rangle\right\rangle_{\phi}^{2}-\left\langle\left\langle\left\langle\gamma_{c c}^{2}\right\rangle\right\rangle\right\rangle_{\phi}^{2}-\left\langle\left\langle\left\langle\gamma_{c c}^{2}\right\rangle\right\rangle\right\rangle_{\phi}^{2}\right\} \geq \frac{1}{2}\left\langle\left\langle\left\langle\gamma_{c c}^{2}+\gamma_{s s}^{2}\right\rangle\right\rangle\right\rangle_{\phi}^{2}
\end{aligned}
$$

For model I we subtract the right argument of (49) from the left argument and find

$$
\begin{aligned}
\mathrm{LA}-\mathrm{RA} & =\frac{1}{2}\left\langle\left\langle\left(\gamma_{c c}-\gamma_{s s}\right)^{2}\right\rangle\right\rangle_{\phi}-\left\langle\left\langle\left\langle\gamma_{c s}^{2}\right\rangle\right\rangle\right\rangle_{\phi}+\sqrt{\left\langle\left\langle\left\langle\gamma_{c s}^{2}\right\rangle\right\rangle\right\rangle_{\phi}^{2}+\frac{1}{4}\left\langle\left\langle\left\langle\gamma_{c c}^{2}-\gamma_{s s}^{2}\right\rangle\right\rangle\right\rangle_{\phi}^{2}} \\
& \geq \frac{1}{2}\left\langle\left\langle\left(\gamma_{c c}-\gamma_{s s}\right)^{2}\right\rangle\right\rangle_{\phi} \geq 0
\end{aligned}
$$

Thus the maximum in (49) is always realized by the left argument. Similarly, upon subtracting the right argument from the left argument in (50), using (51,52), we find

$$
\begin{aligned}
\mathrm{LA}-\mathrm{RA} & =2\left\langle\left\langle\left\langle\gamma_{c s}^{2}\right\rangle\right\rangle\right\rangle_{\phi}+\sqrt{\left\langle\left\langle\left\langle\gamma_{c c}^{2}\right\rangle\right\rangle\right\rangle_{\phi}\left\langle\left\langle\left\langle\gamma_{s s}^{2}\right\rangle\right\rangle\right\rangle_{\phi}}-\left\langle\left\langle\left\langle\gamma_{c c} \gamma_{s s}\right\rangle\right\rangle\right\rangle_{\phi} \\
& \geq \sqrt{\left.\left\langle\left\langle\gamma_{c c}^{2}\right\rangle\right\rangle_{\phi}^{2}\left\langle\left\langle\gamma_{s s}^{2}\right\rangle\right\rangle\right\rangle_{\phi}^{2}}-\frac{1}{2}\left\langle\left\langle\left\langle\gamma_{c c}^{2}+\gamma_{s s}^{2}\right\rangle\right\rangle\right\rangle_{\phi}=\frac{1}{2}(\sqrt{2}-1)\left\langle\left\langle\left\langle\gamma_{c c}^{2}+\gamma_{s s}^{2}\right\rangle\right\rangle_{\phi} \geq 0\right.
\end{aligned}
$$

Hence also the maximum in (50) is realized by the left argument. We conclude that

$$
\begin{aligned}
\text { AT line I : } & {\left[\frac{T}{2 K}\right]^{2}=\frac{1}{2}\left\langle\left\langle\left\langle\gamma_{c c}^{2}+\gamma_{s s}^{2}\right\rangle\right\rangle\right\rangle_{\phi}+\sqrt{\left.\left\langle\left\langle\left\langle\gamma_{c s}^{2}\right\rangle\right\rangle\right\rangle_{\phi}^{2}+\frac{1}{4}\left\langle\left\langle\gamma_{c c}^{2}-\gamma_{s s}^{2}\right\rangle\right\rangle\right\rangle_{\phi}^{2}} } \\
\text { AT line II : } & {\left[\frac{T}{2 K}\right]^{2}=\left\langle\left\langle\left\langle\gamma_{c s}^{2}\right\rangle\right\rangle_{\phi}+\sqrt{\left\langle\left\langle\left\langle\gamma_{c c}^{2}\right\rangle\right\rangle\right\rangle_{\phi}\left\langle\left\langle\left\langle\gamma_{s s}^{2}\right\rangle\right\rangle_{\phi}\right.}\right.}
\end{aligned}
$$

Finally, in reflection symmetric states with $p(\phi)=p(-\phi) \forall \phi$, where we may use identity (45), the replica-symmetric susceptibility (36) is found to be purely real:

$$
\frac{\chi_{\mathrm{RS}}}{\beta}=\frac{1}{2}\left(1-q_{c c}-q_{s s}\right)-\left\langle\left\langle\left\langle\frac{1}{2} \cos (2 \phi)\left(\gamma_{c c}-\gamma_{s s}\right)+\sin (2 \phi) \gamma_{c s}\right)\right\rangle_{\phi}\right.
$$




\subsection{Reflection symmetry breaking transitions}

Assuming reflection symmetry breaking (to states with $Q_{c s} \neq 0$ and/or $q_{c s} \neq 0$ ) to happen via second order transitions, allows us to determine its occurrence by studying the relevant entries of the RS Hessian of the free energy. Such transitions occur when

$$
\operatorname{Det}\left|\begin{array}{cc}
\partial^{2} f\left[Q_{c c, q_{s s}, q_{c c}}\right] / \partial Q_{c s}^{2} & \partial^{2} f\left[Q_{c c, q_{s s}, q_{c c}}\right] / \partial Q_{c s} \partial q_{c s} \\
\partial^{2} f\left[Q_{c c, q_{s s}, q_{c c}}\right] / \partial Q_{c s} \partial q_{c s} & \partial^{2} f\left[Q_{c c, q_{s s}, q_{c c}}\right] / \partial q_{c s}^{2}
\end{array}\right|=0
$$

The relevant matrix elements of the Hessian are given in Appendix A. We can deal with both our two models simultaneously upon defining the variable $\tau \in\{-1,1\}$, where $\tau=1$ for model I and $\tau=-1$ for model II. This allows us to write:

$$
\begin{array}{ll}
\frac{1}{\beta K^{2}} \frac{\partial^{2} f}{\partial Q_{c s}^{2}} & =4 \tau-8(\beta K)^{2} \lambda_{1} \\
\frac{1}{\beta K^{2}} \frac{\partial^{2} f}{\partial q_{c s}^{2}} & =-4 \tau+8(\beta K)^{2} \lambda_{2} \\
\frac{1}{\beta K^{2}} \frac{\partial^{2} f}{\partial Q_{c s} \partial q_{c s}} & =4(\beta K)^{2} \lambda_{3}
\end{array}
$$

They involve

$$
\begin{gathered}
\lambda_{1}=\frac{1}{2}\left\langle\left\langle\left\langle\left\langle\sin ^{2}(2 \theta)\right\rangle_{\star}-\langle\sin (2 \theta)\rangle_{\star}^{2}\right\rangle\right\rangle\right\rangle_{\phi} \\
\lambda_{2}=\left\langle\left\langle\left\langle\left[\left\langle\sin ^{2}(\theta)\right\rangle_{\star}-\langle\sin (\theta)\rangle_{\star}^{2}\right]\left[\left\langle\cos ^{2}(\theta)\right\rangle_{\star}-3\langle\cos (\theta)\rangle_{\star}^{2}\right]\right.\right.\right. \\
+\left[\left\langle\cos ^{2}(\theta)\right\rangle_{\star}-\langle\cos (\theta)\rangle_{\star}^{2}\right]\left[\left\langle\sin ^{2}(\theta)\right\rangle_{\star}-3\langle\sin (\theta)\rangle_{\star}^{2}\right] \\
\left.\left.+2\left[\langle\sin (\theta) \cos (\theta)\rangle_{\star}-\langle\sin (\theta)\rangle_{\star}\langle\cos (\theta)\rangle_{\star}\right]\left[\langle\sin (\theta) \cos (\theta)\rangle_{\star}-3\langle\sin (\theta)\rangle_{\star}\langle\cos (\theta)\rangle_{\star}\right]\right\rangle\right\rangle_{\phi} \\
\lambda_{3}=2\left\langle\left\langle\left\langle\langle\sin (2 \theta) \cos (\theta)\rangle_{\star}\langle\sin (\theta)\rangle_{\star}+\langle\sin (2 \theta) \sin (\theta)\rangle_{\star}\langle\cos (\theta)\rangle_{\star}\right.\right.\right. \\
\left.\left.-2\langle\sin (2 \theta)\rangle_{\star}\langle\sin (\theta)\rangle_{\star}\langle\cos (\theta)\rangle_{\star}\right\rangle\right\rangle_{\phi}
\end{gathered}
$$

Insertion of the second derivatives into (56), using the above short-hands (60, 61, 62 reveals that reflection symmetry breaking transitions are marked by the highest temperature for which the following functions $\sum_{\mathrm{I}, \mathrm{II}}^{\mathrm{ref}}(T)$ become negative:

$$
\begin{array}{ll}
\text { Model I : } & \Sigma_{\mathrm{I}}^{\mathrm{ref}}(T)=(T / K)^{2}-\sqrt{\left(\lambda_{1}-\lambda_{2}\right)^{2}-\lambda_{3}^{2}}-\lambda_{1}-\lambda_{2} \\
\text { Model II : } & \Sigma_{\mathrm{II}}^{\mathrm{ref}}(T)=(T / K)^{2}-\sqrt{\left(\lambda_{1}-\lambda_{2}\right)^{2}-\lambda_{3}^{2}}+\lambda_{1}+\lambda_{2}
\end{array}
$$

Since the $\left\{\lambda_{i}\right\}$ are bounded, expressions (63,64) confirm that reflection symmetry will always be stable for sufficiently high temperatures.

\section{States with global rotation symmetry}

For uniformly distributed pinning angles, i.e. $p(\phi)=(2 \pi)^{-1}$, we may expect the macroscopic state to have global rotation symmetry for sufficiently high temperatures, in addition to reflection symmetry. We therefore inspect the effect of global rotations $\theta_{i} \rightarrow \theta_{i}+\psi$ on the order parameters (within the RS ansatz), with the help of 23.24.25):

$$
\theta_{i}^{\prime}=\theta_{i}+\psi \text { forall } i: \quad q_{c c}^{\prime}+q_{s s}^{\prime}=q_{c c}+q_{s s}
$$


Frustrated coupled oscillators with random fields - October 23rd, 2002

$$
\begin{aligned}
\left(\begin{array}{c}
Q_{c c}^{\prime}-\frac{1}{2} \\
Q_{c s}^{\prime}
\end{array}\right) & =\left(\begin{array}{cc}
\cos (2 \psi) & -\sin (2 \psi) \\
\sin (2 \psi) & \cos (2 \psi)
\end{array}\right)\left(\begin{array}{c}
Q_{c c}-\frac{1}{2} \\
Q_{c s}
\end{array}\right) \\
\left(\begin{array}{cc}
\frac{1}{2}\left(q_{c c}^{\prime}-q_{s s}^{\prime}\right) \\
q_{c s}^{\prime}
\end{array}\right) & =\left(\begin{array}{cc}
\cos (2 \psi) & -\sin (2 \psi) \\
\sin (2 \psi) & \cos (2 \psi)
\end{array}\right)\left(\begin{array}{c}
\frac{1}{2}\left(q_{c c}-q_{s s}\right) \\
q_{c s}
\end{array}\right)
\end{aligned}
$$

Invariance under all global rotations implies $Q_{c c}=\frac{1}{2}, Q_{c s}=q_{c s}=0, q_{c c}=q_{s s}=q$ (rotation-invariance implies reflection-invariance), leaving just one order parameter in rotation invariant states. The invariant manifolds in order parameter space are

$$
\begin{aligned}
& \left(Q_{c c}-\frac{1}{2}\right)^{2}+Q_{c s}^{2}=\epsilon_{1} \\
& \frac{1}{4}\left(q_{c c}-q_{s s}\right)^{2}+q_{c s}^{2}=\epsilon_{2}
\end{aligned}
$$

with the invariant state corresponding to $\epsilon_{1}=\epsilon_{2}=0$. We will also find instances of rotation-invariant states without the pinning field distribution having rotational symmetry. Insertion of $\left\{Q_{c c}=\frac{1}{2}, Q_{c s}=q_{c s}=0, q_{c c}=q_{s s}=q\right\}$ as an ansatz into our $\mathrm{RS}$ order parameter equations reveals that (for nonzero $h$ ) the following condition is necessary and sufficient for the existence of a rotation-invariant solution:

$$
\left\langle\cos ^{2}(\phi)\right\rangle_{\phi}=\left\langle\sin ^{2}(\phi)\right\rangle_{\phi}
$$

Hence, unless explicitly stated we will in this section not assume $p(\phi)$ to be uniform, but rely only on the two properties $p(\phi)=p(-\phi)$ (assumed to hold throughout this paper) and $\langle\cos (2 \phi)\rangle_{\phi}=0$ (to guarantee the existence of a rotation-invariant state).

\subsection{Implications for free energy and order parameters}

For rotation-symmetric states the measures (43,44) become identical:

$$
M(\theta \mid x, y, \phi)=e^{\beta h \cos (\theta-\phi)+2 \beta K \sqrt{q}[x \cos (\theta)+y \sin (\theta)]}
$$

For any value of $\phi$ one can carry out a suitable rotation of the Gaussian variables $(x, y)$ to eliminate $\phi$ from the measure (69), leading to the following general identity for any set of functions $\left\{k_{\ell}\right\}$ (with $\left.\ell=0,1,2, \ldots\right)$ :

$$
\left\langle\left\langle\left\langle k_{0}(\phi) \prod_{\ell>0}\left\langle k_{\ell}(\theta)\right\rangle_{\star}\right\rangle\right\rangle\right\rangle_{\phi}=\left\langle\left\langle\left\langle k_{0}(\phi) \prod_{\ell>0}\left\langle k_{\ell}(\theta+\phi)\right\rangle_{\circ}\right\rangle\right\rangle_{\phi}\right.
$$

where $\langle\ldots\rangle_{\circ}$ refers to averages calculated with the $\phi$-independent measure $M(\theta \mid x, y)=$ $M(\theta \mid x, y, 0)$. As a consequence our subsequent calculations for rotation-invariant states will repeatedly involve various derivatives of the following generating function:

$$
\begin{aligned}
Z[x, y] & =\log \int d \theta e^{\beta h \cos (\theta)+2 \beta K \sqrt{q}[x \cos (\theta)+y \sin (\theta)]} \\
& =\log (2 \pi)+\log I_{0}[\Xi]
\end{aligned}
$$

with the short-hand $\Xi=\beta \sqrt{(h+2 K x \sqrt{q})^{2}+(2 K y \sqrt{q})^{2}}$, and in which $I_{n}[z]$ denotes the $n$-th modified Bessel function 30 . For instance:

$$
\begin{array}{ll}
\frac{1}{2 \beta K \sqrt{q}} \frac{\partial}{\partial x} Z[x, y] & =\langle\cos (\theta)\rangle_{\circ} \\
\frac{1}{2 \beta K \sqrt{q}} \frac{\partial}{\partial y} Z[x, y] & =\langle\sin (\theta)\rangle_{\circ} \\
\frac{1}{(2 \beta K \sqrt{q})^{2}} \frac{\partial^{2}}{\partial x^{2}} Z[x, y] & =\left\langle\cos ^{2}(\theta)\right\rangle_{\circ}-\langle\cos (\theta)\rangle_{\circ}^{2}
\end{array}
$$


Frustrated coupled oscillators with random fields - October 23rd, 2002

$$
\begin{aligned}
\frac{1}{(2 \beta K \sqrt{q})^{2}} \frac{\partial^{2}}{\partial y^{2}} Z[x, y]= & \left\langle\sin ^{2}(\theta)\right\rangle_{\circ}-\langle\sin (\theta)\rangle_{\circ}^{2} \\
\frac{1}{(2 \beta K \sqrt{q})^{2}} \frac{\partial^{2}}{\partial x \partial y} Z[x, y]= & \langle\sin (\theta) \cos (\theta)\rangle_{\circ}-\langle\sin (\theta)\rangle_{\circ}\langle\cos (\theta)\rangle_{\circ} \\
\frac{1}{(2 \beta K \sqrt{q})^{3}} \frac{\partial^{3}}{\partial x^{2} \partial y} Z[x, y]= & \left\langle\sin (\theta) \cos ^{2}(\theta)\right\rangle_{\circ}-\langle\sin (\theta)\rangle_{\circ}\left\langle\cos ^{2}(\theta)\right\rangle_{\circ} \\
& -\langle\sin (2 \theta)\rangle_{\circ}\langle\cos (\theta)\rangle_{\circ}+2\langle\sin (\theta)\rangle_{\circ}\langle\cos (\theta)\rangle_{\circ}^{2} \\
\frac{1}{(2 \beta K \sqrt{q})^{3}} \frac{\partial^{3}}{\partial x \partial y^{2}} Z[x, y]= & \left\langle\sin ^{2}(\theta) \cos (\theta)\right\rangle_{\circ}-\langle\cos (\theta)\rangle_{\circ}\left\langle\sin ^{2}(\theta)\right\rangle_{\circ} \\
& -\langle\sin (2 \theta)\rangle_{\circ}\langle\sin (\theta)\rangle_{\circ}+2\langle\cos (\theta)\rangle_{\circ}\langle\sin (\theta)\rangle_{\circ}^{2}
\end{aligned}
$$

In order to work out the remaining order parameter equation for $q$ we apply the identity $(70)$ to $k_{1}(\theta)=k_{2}(\theta)=\cos (\theta)$ and to $k_{1}(\theta)=k_{2}(\theta)=\sin (\theta)$, giving

$$
\begin{aligned}
& \left\langle\left\langle\left\langle\langle\cos (\theta)\rangle_{\star}^{2}\right\rangle\right\rangle\right\rangle_{\phi}=\left\langle\cos ^{2}(\phi)\right\rangle_{\phi}\left\langle\left\langle\langle\cos (\theta)\rangle_{\circ}^{2}\right\rangle\right\rangle+\left\langle\sin ^{2}(\phi)\right\rangle_{\phi}\left\langle\left\langle\langle\sin (\theta)\rangle_{\circ}^{2}\right\rangle\right\rangle \\
& \left\langle\left\langle\left\langle\langle\sin (\theta)\rangle_{\star}^{2}\right\rangle\right\rangle\right\rangle_{\phi}=\left\langle\sin ^{2}(\phi)\right\rangle_{\phi}\left\langle\left\langle\langle\cos (\theta)\rangle_{\circ}^{2}\right\rangle\right\rangle+\left\langle\cos ^{2}(\phi)\right\rangle_{\phi}\left\langle\left\langle\langle\sin (\theta)\rangle_{\circ}^{2}\right\rangle\right\rangle
\end{aligned}
$$

Thus the remaining order parameter $q$ is, for both models, to be solved from

$$
\begin{aligned}
q & =\frac{1}{2} \int D x D y\left\langle\langle\cos (\theta)\rangle_{\star}^{2}+\langle\sin (\theta)\rangle_{\star}^{2}\right\rangle_{\phi} \\
& =\frac{1}{2} \int D x D y\left[\langle\cos (\theta)\rangle_{\circ}^{2}+\langle\sin (\theta)\rangle_{\circ}^{2}\right]
\end{aligned}
$$

Using properties of modified Bessel functions, such as $\frac{d}{d z} I_{n}[z]=\frac{1}{2}\left(I_{n+1}[z]+I_{n-1}[z]\right)$ and $I_{n}[z]=(z / 2 n)\left(I_{n-1}[z]-I_{n+1}[z]\right)$, the two relevant expressions (72,73) give

$$
\begin{aligned}
& \langle\cos (\theta)\rangle_{\circ}=\frac{\beta(h+2 K x \sqrt{q})}{\Xi} \frac{I_{1}[\Xi]}{I_{0}[\Xi]} \\
& \langle\sin (\theta)\rangle_{\circ}=\frac{\beta(2 K y \sqrt{q})}{\Xi} \frac{I_{1}[\Xi]}{I_{0}[\Xi]}
\end{aligned}
$$

After writing $(x, y)$ in polar coordinates, one then finds (31) reducing to

$$
q=\int_{0}^{\pi} \frac{d \psi}{2 \pi} \int_{0}^{\infty} d r r e^{-\frac{1}{2} r^{2}}\left\{\frac{I_{1}[\Xi(r, \psi)]}{I_{0}[\Xi(r, \psi)]}\right\}^{2}
$$

with

$$
\Xi(r, \psi)=\beta \sqrt{4 q K^{2} r^{2}+h^{2}+4 K h r \sqrt{q} \cos (\psi)}
$$

Inserting $Q_{c s}=q_{c s}=0, Q_{c c}=\frac{1}{2}$, and $q_{c c}=q_{s s}=q$ into expressions $30,32,33$, shows that for rotation-symmetric states and for both models I and II the disorderaveraged free energy per oscillator equals $\bar{f}=\operatorname{extr}_{q} f[q]-\frac{1}{\beta} \log (2 \pi)$, with

$$
f[q]=-2 \beta K^{2}\left(q-\frac{1}{2}\right)^{2}-\frac{1}{\beta} \int_{0}^{\pi} \frac{d \psi}{\pi} \int_{0}^{\infty} d r r e^{-\frac{1}{2} r^{2}} \log I_{0}[\Xi(r, \psi)]
$$

According to (34, 35) the function $f[q]$ is in both cases to be maximized, so the replica symmetric rotation-invariant ground state would be $q=\frac{1}{2}$. 


\subsection{Implications for covariances, AT lines, and RS susceptibility}

Various terms involving the covariances $\gamma_{\star \star}(28,29)$ can be simplified via (70). We define $\bar{\gamma}_{\star \star}=\left.\gamma_{\star \star}\right|_{\phi=0}$ (i.e. as calculated with the $\phi=0$ averages $\langle\ldots\rangle_{\circ}$ ). For instance, focusing on the terms occurring in (54,54) and using (68):

$$
\begin{aligned}
& \left\langle\left\langle\left\langle\gamma_{c c}^{2}\right\rangle\right\rangle\right\rangle_{\phi}=\left\langle\left\langle\frac{1}{4}\left(\bar{\gamma}_{c c}+\bar{\gamma}_{s s}\right)^{2}+\bar{\gamma}_{c s}^{2}\right\rangle\right\rangle+\left\langle\cos ^{2}(2 \phi)\right\rangle_{\phi}\left\langle\left\langle\frac{1}{4}\left(\bar{\gamma}_{c c}-\bar{\gamma}_{s s}\right)^{2}-\bar{\gamma}_{c s}^{2}\right\rangle\right\rangle \\
& \left\langle\left\langle\left\langle\gamma_{s s}^{2}\right\rangle\right\rangle\right\rangle_{\phi}=\left\langle\left\langle\frac{1}{4}\left(\bar{\gamma}_{c c}+\bar{\gamma}_{s s}\right)^{2}+\bar{\gamma}_{c s}^{2}\right\rangle\right\rangle+\left\langle\cos ^{2}(2 \phi)\right\rangle_{\phi}\left\langle\left\langle\frac{1}{4}\left(\bar{\gamma}_{c c}-\bar{\gamma}_{s s}\right)^{2}-\bar{\gamma}_{c s}^{2}\right\rangle\right\rangle \\
& \left\langle\left\langle\left\langle\gamma_{c s}^{2}\right\rangle\right\rangle\right\rangle_{\phi}=\left\langle\sin ^{2}(2 \phi)\right\rangle_{\phi}\left\langle\left\langle\left\langle\frac{1}{4}\left(\bar{\gamma}_{c c}-\bar{\gamma}_{s s}\right)^{2}\right\rangle\right\rangle+\left\langle\cos ^{2}(2 \phi)\right\rangle_{\phi}\left\langle\left\langle\bar{\gamma}_{c s}^{2}\right\rangle\right\rangle\right. \\
& \frac{1}{4}\left\langle\left\langle\left\langle\left(\gamma_{c c}-\gamma_{s s}\right)^{2}\right\rangle\right\rangle\right\rangle_{\phi}=\left\langle\sin ^{2}(2 \phi)\right\rangle_{\phi}\left\langle\left\langle\bar{\gamma}_{c s}^{2}\right\rangle+\left\langle\cos ^{2}(2 \phi)\right\rangle_{\phi}\left\langle\left\langle\frac{1}{4}\left(\bar{\gamma}_{c c}-\bar{\gamma}_{s s}\right)^{2}\right\rangle\right\rangle\right.
\end{aligned}
$$

From (74, 75,76$)$, in turn, one obtains explicit expressions for the required $\left\{\bar{\gamma}_{\star \star}\right\}$ :

$$
\begin{aligned}
& \bar{\gamma}_{c c}=\frac{1}{2}-\frac{I_{2}[\Xi]}{2 I_{0}[\Xi]}+\frac{\beta^{2}(h+2 K x \sqrt{q})^{2}}{\Xi^{2}}\left\{\frac{I_{2}[\Xi]}{I_{0}[\Xi]}-\frac{I_{1}^{2}[\Xi]}{I_{0}^{2}[\Xi]}\right\} \\
& \bar{\gamma}_{s s}=\frac{1}{2}-\frac{I_{2}[\Xi]}{2 I_{0}[\Xi]}+\frac{\beta^{2}(2 K y \sqrt{q})^{2}}{\Xi^{2}}\left\{\frac{I_{2}[\Xi]}{I_{0}[\Xi]}-\frac{I_{1}^{2}[\Xi]}{I_{0}^{2}[\Xi]}\right\} \\
& \bar{\gamma}_{c s}=\frac{\beta^{2}(h+2 K x \sqrt{q})(2 K y \sqrt{q})}{\Xi^{2}}\left\{\frac{I_{2}[\Xi]}{I_{0}[\Xi]}-\frac{I_{1}^{2}[\Xi]}{I_{0}^{2}[\Xi]}\right\}
\end{aligned}
$$

Combination of the above results with $(53,54)$ gives the equations for the AT lines. We note that for rotation invariant states the two expressions (53,54) become identical:

$$
\begin{aligned}
{\left[\frac{T}{K}\right]^{2} } & =4\left\langle\left\langle\frac{1}{2} \bar{\gamma}_{c c}^{2}+\frac{1}{2} \bar{\gamma}_{s s}^{2}+\bar{\gamma}_{c s}^{2}\right\rangle\right. \\
& =\int D x D y\left\{\left[\frac{I_{2}[\Xi]}{I_{0}[\Xi]}-\frac{I_{1}^{2}[\Xi]}{I_{0}^{2}[\Xi]}\right]^{2}+\left[1-\frac{I_{1}^{2}[\Xi]}{I_{0}^{2}[\Xi]}\right]^{2}\right\}
\end{aligned}
$$

After transformation of the Gaussian variables to polar coordinates, this gives for the AT lines of our two models the appealing result

$$
\left[\frac{T}{K}\right]^{2}=\int_{0}^{\pi} \frac{d \psi}{\pi} \int_{0}^{\infty} d r r e^{-\frac{1}{2} r^{2}}\left\{\left[\frac{I_{2}[\Xi(r, \psi)]}{I_{0}[\Xi(r, \psi)]}-\frac{I_{1}^{2}[\Xi(r, \psi)]}{I_{0}^{2}[\Xi(r, \psi)]}\right]^{2}+\left[1-\frac{I_{1}^{2}[\Xi(r, \psi)]}{I_{0}^{2}[\Xi(r, \psi)]}\right]^{2}\right\}
$$

In a similar manner we work out expression (55) for rotation-invariant states:

$$
\begin{aligned}
\frac{\chi_{\mathrm{RS}}}{\beta}= & \left.\frac{1}{2}(1-2 q)-\left\langle\left\langle\frac{1}{2} \cos (2 \phi)\left(\gamma_{c c}-\gamma_{s s}\right)+\sin (2 \phi) \gamma_{c s}\right\rangle\right\rangle\right\rangle_{\phi} \\
= & \frac{1}{2}(1-2 q)-\frac{1}{2} \int D x D y\left(\bar{\gamma}_{c c}-\bar{\gamma}_{s s}\right) \\
= & \frac{1}{2}-q-\int_{0}^{\pi} \frac{d \psi}{2 \pi} \int_{0}^{\infty} d r r e^{-\frac{1}{2} r^{2}}\left[\frac{I_{2}[\Xi(r, \psi)]}{I_{0}[\Xi(r, \psi)]}-\frac{I_{1}^{2}[\Xi(r, \psi)]}{I_{0}^{2}[\Xi(r, \psi)]}\right] \\
& \times\left[\frac{h^{2}+4 K^{2} r^{2} q \cos (2 \psi)+4 h K r \sqrt{q} \cos (\psi)}{h^{2}+4 K^{2} r^{2} q+4 h K r \sqrt{q} \cos (\psi)}\right]
\end{aligned}
$$

with the abbreviation (85). Note that $\lim _{h \rightarrow 0} \Xi(r, \psi)=2 \beta K r \sqrt{q}$ (i.e. independent of $\psi$ ), so that for rotation invariant states one has

$$
\lim _{h \rightarrow 0} \chi_{\mathrm{RS}}=\beta\left(\frac{1}{2}-q\right)
$$




\subsection{Rotation symmetry breaking transitions}

According to (66, 67) we can study rotation symmetry-breaking bifurcations most conveniently after the following transformation of the order parameters:

$$
\begin{array}{ll}
Q_{c c}=\frac{1}{2}+\epsilon_{1} \cos \left(\omega_{1}\right), & Q_{c s}=\epsilon_{1} \sin \left(\omega_{1}\right) \\
q_{c c}=q+\epsilon_{2} \cos \left(\omega_{2}\right), & q_{s s}=q-\epsilon_{2} \cos \left(\omega_{2}\right),
\end{array}
$$

Reflection symmetry breaking transitions are already marked by (63,64), so we restrict ourselves to rotation symmetry breaking transitions which preserve reflection symmetry, i.e. $Q_{c s}=q_{c s}=0$. Thus we put $\omega_{1}=\omega_{2}=0$ and focus on

$$
Q_{c c}=\frac{1}{2}+\epsilon_{1}, \quad Q_{c s}=q_{c s}=0, \quad q_{c c}=q+\epsilon_{2}, \quad q_{s s}=q-\epsilon_{2}
$$

Our interest is in continuous bifurcations of $\epsilon_{1} \neq 0$ and/or $\epsilon_{2} \neq 0$. We expand the free energy per oscillator $f[\ldots]$ to be extremized (30) around the rotation invariant solution, using the second order derivatives in Appendix A. Due to reflection symmetry, only those second derivatives with an even total number of 's' subscripts in the two corresponding order parameters can be nonzero. This gives for $0 \ll \epsilon_{1}, \epsilon_{2} \ll 1$ (with $f_{\mathrm{RI}}[\ldots]$ denoting the free energy of the rotation invariant state):

$$
\begin{aligned}
f[\ldots]-f_{\mathrm{RI}}[\ldots]= & \frac{1}{2} \epsilon_{1}^{2} \frac{\partial^{2} f}{\partial Q_{c c}^{2}}+\frac{1}{2} \epsilon_{2}^{2}\left\{\frac{\partial^{2} f}{\partial q_{c c}^{2}}+\frac{\partial^{2} f}{\partial q_{s s}^{2}}-2 \frac{\partial^{2} f}{\partial q_{c c} \partial q_{s s}}\right\} \\
& +\epsilon_{1} \epsilon_{2}\left\{\frac{\partial^{2} f}{\partial Q_{c c} \partial q_{c c}}-\frac{\partial^{2} f}{\partial Q_{c c} \partial q_{s s}}\right\}+\mathcal{O}\left(\epsilon^{3}\right)
\end{aligned}
$$

Hence the continuous rotation symmetry breaking transitions are marked by

$$
\operatorname{Det}\left|\begin{array}{cc}
\partial^{2} f / \partial Q_{c c}^{2} & \partial^{2} f / \partial Q_{c c} \partial q_{c c}-\partial^{2} f / \partial Q_{c c} \partial q_{s s} \\
\partial^{2} f / \partial Q_{c c} \partial q_{c c}-\partial^{2} f / \partial Q_{c c} \partial q_{s s} & \partial^{2} f / \partial q_{c c}^{2}+\partial^{2} f / \partial q_{s s}^{2}-2 \partial^{2} f / \partial q_{c c} \partial q_{s s}
\end{array}\right|=0
$$

We work out the second derivatives for rotation invariant states (where the effective measures of the two models are identical), using (70). Given our requirements $p(\phi)=p(-\phi)$ and $\langle\cos (2 \phi)\rangle_{\phi}$, these are found to depend on the choice made for the pinning field distribution only through $\langle\cos (4 \phi)\rangle_{\phi}$. Again we deal with both models simultaneously upon defining $\tau \in\{-1,1\}$, where $\tau=1$ for model I and $\tau=-1$ for model II. This allows us to write:

$$
\begin{array}{ll}
\frac{1}{\beta K^{2}} \frac{\partial^{2} f}{\partial Q_{c c}^{2}} & =4 \tau-8(\beta K)^{2} \rho_{1} \\
\frac{1}{\beta K^{2}}\left\{\frac{\partial^{2} f}{\partial q_{c c}^{2}}+\frac{\partial^{2} f}{\partial q_{s s}^{2}}-2 \frac{\partial^{2} f}{\partial q_{c c} \partial q_{s s}}\right\} & =-4 \tau+8(\beta K)^{2} \rho_{2} \\
\frac{1}{\beta K^{2}}\left\{\frac{\partial^{2} f}{\partial Q_{c c} \partial q_{c c}}-\frac{\partial^{2} f}{\partial Q_{c c} q_{s s}}\right\} & =4(\beta K)^{2} \rho_{3}
\end{array}
$$

where straightforward but tedious bookkeeping shows the quantities $\left\{\rho_{1}, \rho_{2}, \rho_{3}\right\}$ to be

$\rho_{1}=\frac{1}{4}\left\langle\left\langle 1-\left\langle\cos (2 \theta\rangle_{\circ}^{2}-\left\langle\sin (2 \theta\rangle_{\circ}^{2}\right\rangle\right\rangle+\frac{1}{4}\langle\cos (4 \phi)\rangle_{\phi}\left\langle\left\langle\langle\cos (4 \theta)\rangle_{\circ}-\left\langle\cos (2 \theta\rangle_{\circ}^{2}+\left\langle\sin (2 \theta\rangle_{\circ}^{2}\right\rangle\right\rangle\right.\right.\right.\right.$

$\rho_{2}=\left\langle\left\langle\left[\left\langle\cos ^{2}(\theta)\right\rangle_{\circ}-\langle\cos (\theta)\rangle_{0}^{2}\right]\left[\left\langle\cos ^{2}(\theta)\right\rangle_{\circ}-3\langle\cos (\theta)\rangle_{\circ}^{2}\right]\right.\right.$ 
Frustrated coupled oscillators with random fields - October 23rd, 2002

$$
\begin{gathered}
+\left[\left\langle\sin ^{2}(\theta)\right\rangle_{\circ}-\langle\sin (\theta)\rangle_{\circ}^{2}\right]\left[\left\langle\sin ^{2}(\theta)\right\rangle_{\circ}-3\langle\sin (\theta)\rangle_{\circ}^{2}\right] \\
\left.\left.-\frac{1}{2}\left[\langle\cos (2 \theta)\rangle_{\circ}-\langle\cos (\theta)\rangle_{\circ}^{2}+\langle\sin (\theta)\rangle_{\circ}^{2}\right]\left[\langle\cos (2 \theta)\rangle_{\circ}-3\langle\cos (\theta)\rangle_{\circ}^{2}+3\langle\sin (\theta)\rangle_{\circ}^{2}\right]\right\rangle\right\rangle \\
+\langle\cos (4 \phi)\rangle_{\phi}\left\langle\left\langle\frac{1}{2}\left[\langle\cos (2 \theta)\rangle_{\circ}-\langle\cos (\theta)\rangle_{\circ}^{2}+\langle\sin (\theta)\rangle_{\circ}^{2}\right]\left[\langle\cos (2 \theta)\rangle_{\circ}-3\langle\cos (\theta)\rangle_{\circ}^{2}+3\langle\sin (\theta)\rangle_{0}^{2}\right]\right.\right. \\
\left.-2\left[\langle\sin (\theta) \cos (\theta)\rangle_{\circ}-\langle\sin (\theta)\rangle_{\circ}\langle\cos (\theta)\rangle_{\circ}\right]\left[\langle\sin (\theta) \cos (\theta)\rangle_{\circ}-3\langle\sin (\theta)\rangle_{\circ}\langle\cos (\theta)\rangle_{\circ}\right]\right\rangle \\
\rho_{3}=2\left\langle\left\langle\left\langle\sin ^{2}(\theta)\right\rangle_{\circ}\langle\cos (\theta)\rangle_{\circ}^{2}+\left\langle\cos ^{2}(\theta)\right\rangle_{\circ}\langle\sin (\theta)\rangle_{\circ}^{2}-\langle\sin (2 \theta)\rangle_{\circ}\langle\sin (\theta)\rangle_{\circ}\langle\cos (\theta)\rangle_{\circ}\right\rangle\right\rangle \\
+2\langle\cos (4 \phi)\rangle_{\phi}\left\langle\left\langle\left\langle\sin ^{2}(\theta)\right\rangle_{\circ}\langle\cos (\theta)\rangle_{\circ}^{2}+\left\langle\cos ^{2}(\theta)\right\rangle_{\circ}\langle\sin (\theta)\rangle_{\circ}^{2}+\langle\sin (2 \theta)\rangle_{\circ}\langle\sin (\theta)\rangle_{\circ}\langle\cos (\theta)\rangle_{\circ}\right.\right. \\
\left.\left.-2\left\langle\sin ^{2}(\theta) \cos (\theta)\right\rangle_{\circ}\langle\cos (\theta)\rangle_{\circ}-2\left\langle\cos ^{2}(\theta) \sin (\theta)\right\rangle_{\circ}\langle\sin (\theta)\rangle_{\circ}\right\rangle\right\rangle
\end{gathered}
$$

Insertion of the second derivatives into (97), using (101,102,103), shows that rotation symmetry breaking transitions which preserve reflection symmetry are marked by the highest temperature for which the following functions become negative:

$$
\begin{array}{ll}
\text { Model I : } & \Sigma_{\mathrm{I}}^{\mathrm{rot}}(T)=(T / K)^{2}-\sqrt{\left(\rho_{1}-\rho_{2}\right)^{2}-\rho_{3}^{2}}-\rho_{1}-\rho_{2} \\
\text { Model II : } & \Sigma_{\mathrm{II}}^{\mathrm{rot}}(T)=(T / K)^{2}-\sqrt{\left(\rho_{1}-\rho_{2}\right)^{2}-\rho_{3}^{2}}+\rho_{1}+\rho_{2}
\end{array}
$$

What remains is to work out (101, 102, 103). For most terms we can use $71,72-78,91$. 93). One further object in $\rho_{1},\left\langle\left\langle\langle\cos (4 \theta)\rangle_{\circ}\right\rangle\right\rangle$, can be calculated directly as follows

$$
\begin{aligned}
\left\langle\left\langle\langle\cos (4 \theta)\rangle_{0}\right\rangle\right\rangle & =\left\langle\left\langle\frac{\int d \theta \cos \left(4 \theta+4 \operatorname{atan}\left[\frac{2 K y \sqrt{q}}{h+2 K x \sqrt{q}}\right]\right) e^{\Xi \cos (\theta)}}{2 \pi I_{0}(\Xi)}\right\rangle\right\rangle \\
& =\left\langle\left\langle\cos \left(4 \operatorname{atan}\left[\frac{2 K y \sqrt{q}}{h+2 K x \sqrt{q}}\right]\right) \frac{I_{4}[\Xi]}{I_{0}[\Xi]}\right\rangle\right. \\
& =\left\langle\left\langle\left[1-\frac{8 \beta^{4}(2 K y \sqrt{q})^{2}(h+2 K x \sqrt{q})^{2}}{\Xi^{4}}\right] \frac{I_{4}[\Xi]}{I_{0}[\Xi]}\right\rangle\right.
\end{aligned}
$$

The final result of the exercise is, after transformation of $(x, y)$ to polar coordinates:

$$
\begin{aligned}
& \rho_{1}=\frac{1}{4} \int_{0}^{\infty} d r r e^{-\frac{1}{2} r^{2}} \int_{0}^{\pi} \frac{d \psi}{\pi}\left\{1-\frac{I_{2}^{2}[\Xi(r, \psi)]}{I_{0}^{2}[\Xi(r, \psi)]}\right\} \\
&+\frac{1}{4}\langle\cos (4 \phi)\rangle_{\phi} \int_{0}^{\infty} d r r e^{-\frac{1}{2} r^{2}} \int_{0}^{\pi} \frac{d \psi}{\pi}\left\{\frac{I_{4}[\Xi(r, \psi)]}{I_{0}[\Xi(r, \psi)]}-\frac{I_{2}^{2}[\Xi(r, \psi)]}{I_{0}^{2}[\Xi(r, \psi)]}\right\} \\
& \times\left\{1-\frac{8 \beta^{4}[2 K r \sqrt{q} \sin (\psi)]^{2}[h+2 K r \sqrt{q} \cos (\psi)]^{2}}{\Xi^{4}(r, \psi)}\right\} \\
& \rho_{2}=\frac{1}{2} \int_{0}^{\infty} d r r e^{-\frac{1}{2} r^{2}} \int_{0}^{\pi} \frac{d \psi}{\pi}\left\{1-\frac{I_{1}^{2}[\Xi(r, \psi)]}{I_{0}^{2}[\Xi(r, \psi)]}\right\}\left\{1-\frac{3 I_{1}^{2}[\Xi(r, \psi)]}{I_{0}^{2}[\Xi(r, \psi)]}\right\} \\
&+\frac{1}{2}\langle\cos (4 \phi)\rangle_{\phi} \int_{0}^{\infty} d r r e^{-\frac{1}{2} r^{2}} \int_{0}^{\pi} \frac{d \psi}{\pi}\left\{\frac{I_{2}[\Xi(r, \psi)]}{I_{0}[\Xi(r, \psi)]}-\frac{I_{1}^{2}[\Xi(r, \psi)]}{I_{0}^{2}[\Xi(r, \psi)]}\left\{\frac{I_{2}[\Xi(r, \psi)]}{I_{0}[\Xi(r, \psi)]}-\frac{3 I_{1}^{2}[\Xi(r, \psi)]}{I_{0}^{2}[\Xi(r, \psi)]}\right\}\right. \\
& \times\left\{1-\frac{8 \beta^{4}[2 K r \sqrt{q} \sin (\psi)]^{2}[h+2 K r \sqrt{q} \cos (\psi)]^{2}}{\Xi^{4}(r, \psi)}\right\}
\end{aligned}
$$




$$
\begin{aligned}
& \rho_{3}=\int_{0}^{\infty} d r r e^{-\frac{1}{2} r^{2}} \int_{0}^{\pi} \frac{d \psi}{\pi}\left\{1-\frac{I_{2}[\Xi(r, \psi)]}{I_{0}[\Xi(r, \psi)]}\right\} \frac{I_{1}^{2}[\Xi(r, \psi)]}{I_{0}^{2}[\Xi(r, \psi)]} \\
&+\langle\cos (4 \phi)\rangle_{\phi} \int_{0}^{\infty} d r r e^{-\frac{1}{2} r^{2}} \int_{0}^{\pi} \frac{d \psi}{\pi}\left\{1-\frac{I_{2}[\Xi(r, \psi)]}{I_{0}[\Xi(r, \psi)]}\right\}\left\{\frac{I_{1}^{2}[\Xi(r, \psi)]}{I_{0}^{2}[\Xi(r, \psi)]}-\frac{2 I_{2}[\Xi(r, \psi)]}{I_{0}[\Xi(r, \psi)]}\right\} \\
& \times\left\{1-\frac{8 \beta^{4}[2 K r \sqrt{q} \sin (\psi)]^{2}[h+2 K r \sqrt{q} \cos (\psi)]^{2}}{\Xi^{4}(r, \psi)}\right\}
\end{aligned}
$$

The general identity $I_{n+1}[z]=I_{n-1}[z]+n I_{n}[z]\left(I_{1}[z]-I_{0}[z]\right) / I_{1}[z]$ allows us to express any $I_{n>2}[z]$ in terms of the trio $\left\{I_{0}[z], I_{1}[z], I_{2}[z]\right\}$. Since the $\left\{\rho_{i}\right\}$ are bounded, expressions (104, 105) confirm that, if rotation invariant states exist (i.e. if $\left.\langle\cos (2 \phi)\rangle_{\phi}=0\right)$, rotation symmetry will be stable for sufficiently large temperatures.

To round off our discussion we return to reflection symmetry breaking transitions. We work out (60, 61, 62 $)$ for rotation invariant states, and find (after a lengthy but straightforward calculation) that

$$
\begin{aligned}
& \lambda_{1}=\frac{1}{4} \int_{0}^{\infty} d r r e^{-\frac{1}{2} r^{2}} \int_{0}^{\pi} \frac{d \psi}{\pi}\left\{1-\frac{I_{2}^{2}[\Xi(r, \psi)]}{I_{0}^{2}[\Xi(r, \psi)]}\right\} \\
& -\frac{1}{4}\langle\cos (4 \phi)\rangle_{\phi} \int_{0}^{\infty} d r r e^{-\frac{1}{2} r^{2}} \int_{0}^{\pi} \frac{d \psi}{\pi}\left\{\frac{I_{4}[\Xi(r, \psi)]}{I_{0}[\Xi(r, \psi)]}-\frac{I_{2}^{2}[\Xi(r, \psi)]}{I_{0}^{2}[\Xi(r, \psi)]}\right\} \\
& \times\left\{1-\frac{8 \beta^{4}[2 K r \sqrt{q} \sin (\psi)]^{2}[h+2 K r \sqrt{q} \cos (\psi)]^{2}}{\Xi^{4}(r, \psi)}\right\} \\
& \lambda_{2}=\frac{1}{2} \int_{0}^{\infty} d r r e^{-\frac{1}{2} r^{2}} \int_{0}^{\pi} \frac{d \psi}{\pi}\left\{1-\frac{I_{1}^{2}[\Xi(r, \psi)]}{I_{0}^{2}[\Xi(r, \psi)]}\right\}\left\{1-\frac{3 I_{1}^{2}[\Xi(r, \psi)]}{I_{0}^{2}[\Xi(r, \psi)]}\right\} \\
& -\frac{1}{2}\langle\cos (4 \phi)\rangle_{\phi} \int_{0}^{\infty} d r r e^{-\frac{1}{2} r^{2}} \int_{0}^{\pi} \frac{d \psi}{\pi}\left\{\frac{I_{2}[\Xi(r, \psi)]}{I_{0}[\Xi(r, \psi)]}-\frac{I_{1}^{2}[\Xi(r, \psi)]}{I_{0}^{2}[\Xi(r, \psi)]}\right\}\left\{\frac{I_{2}[\Xi(r, \psi)]}{I_{0}[\Xi(r, \psi)]}-\frac{3 I_{1}^{2}[\Xi(r, \psi)]}{I_{0}^{2}[\Xi(r, \psi)]}\right\} \\
& \times\left\{1-\frac{8 \beta^{4}[2 K r \sqrt{q} \sin (\psi)]^{2}[h+2 K r \sqrt{q} \cos (\psi)]^{2}}{\Xi^{4}(r, \psi)}\right\} \\
& \lambda_{3}=\int_{0}^{\infty} d r r e^{-\frac{1}{2} r^{2}} \int_{0}^{\pi} \frac{d \psi}{\pi}\left\{1-\frac{I_{2}[\Xi(r, \psi)]}{I_{0}[\Xi(r, \psi)]}\right\} \frac{I_{1}^{2}[\Xi(r, \psi)]}{I_{0}^{2}[\Xi(r, \psi)]} \\
& -\langle\cos (4 \phi)\rangle_{\phi} \int_{0}^{\infty} d r r e^{-\frac{1}{2} r^{2}} \int_{0}^{\pi} \frac{d \psi}{\pi}\left\{1-\frac{I_{2}[\Xi(r, \psi)]}{I_{0}[\Xi(r, \psi)]}\right\}\left\{\frac{I_{1}^{2}[\Xi(r, \psi)]}{I_{0}^{2}[\Xi(r, \psi)]}-\frac{2 I_{2}[\Xi(r, \psi)]}{I_{0}[\Xi(r, \psi)]}\right\} \\
& \times\left\{1-\frac{8 \beta^{4}[2 K r \sqrt{q} \sin (\psi)]^{2}[h+2 K r \sqrt{q} \cos (\psi)]^{2}}{\Xi^{4}(r, \psi)}\right\}
\end{aligned}
$$

Apparently, for rotation-invariant states we obtain the trio $\left\{\lambda_{1}, \lambda_{2}, \lambda_{3}\right\}$ from $\left\{\rho_{1}, \rho_{2}, \rho_{3}\right\}$ by making in the latter the replacement $\langle\cos (4 \phi)\rangle_{\phi} \rightarrow-\langle\cos (4 \phi)\rangle_{\phi}$ :

$$
\lambda_{i}\left(\langle\cos (4 \phi)\rangle_{\phi}\right)=\rho_{i}\left(-\langle\cos (4 \phi)\rangle_{\phi}\right) \quad i=1,2,3
$$

The same is true for the expressions (63, 64) for the reflection symmetry breaking transition lines, which can be obtained by making the substitution $\langle\cos (4 \phi)\rangle_{\phi} \rightarrow$ $-\langle\cos (4 \phi)\rangle_{\phi}$ in the two expressions (104,105) for those rotation symmetry breaking transitions which leave reflection symmetry intact. 


\section{Solution for specific choices for the pinning field statistics}

We now work out our results for specific choices of the pinning field distribution $p(\phi)$ with decreasing levels of symmetry, restricting ourselves (as throughout this paper) to those with $p(\phi)=p(-\phi)$. We start with the benchmark case $h=0$, i.e. absent pinning fields. Wherever possible we have tested our theoretical predictions against extensive numerical simulations. Here the Langevin dynamics defined by equation (3) was iterated with a stochastic Euler method with time step $\Delta t=10^{-3}$, for systems of size either $N=400$ (with the advantage of better equilibration within experimentally accessible time-scales) or $N=800$ (with the advantage of reduced finite size effects). All simulation results shown are averages over 10 experiments.

\subsection{Absent pinning fields}

For $h=0$ the natural solution is the one with full rotational symmetry. Given the RS ansatz we are left with a single order parameter, $q$, and the two measures (26,27) become identical. Insertion of $h=0$ into (84) and (866) immediately leads us to

$$
\begin{aligned}
& q=\frac{1}{2} \int_{0}^{\infty} d r r e^{-\frac{1}{2} r^{2}} \frac{I_{1}^{2}[\Xi(r)]}{I_{0}^{2}[\Xi(r)]} \\
& \bar{f}=\max _{q}\left\{-2 \beta K^{2}\left(q-\frac{1}{2}\right)^{2}-\frac{1}{\beta} \int_{0}^{\infty} d r r e^{-\frac{1}{2} r^{2}} \log I_{0}[\Xi(r)]-\frac{1}{\beta} \log (2 \pi)\right\}
\end{aligned}
$$

with $\Xi(r)=2 \beta K \sqrt{q}$. Expanding the right-hand side of (113) gives $\operatorname{RHS}(q)=$ $(\beta K)^{2} q-4(\beta K)^{4} q^{2}+\mathcal{O}\left(q^{3}\right)$. There is a second order transition at $T_{c}=K$ from a paramagnetic state $(q=0)$ to an ordered state $(q>0)$, and no evidence for firstorder transitions. As the temperature is lowered further, $q$ increases monotonically towards its maximum value at $T=0$, as $q=\frac{1}{2}-T \sqrt{\pi} / 4 K+\mathcal{O}\left(T^{2}\right)$. Close to the critical point we can expand $q$ in powers of $\tau=1-T / T_{c}$ and find $q=\frac{1}{2} \tau+\mathcal{O}\left(\tau^{2}\right)$ $(\tau \downarrow 0)$. All this is in perfect agreement with the results obtained earlier for Gaussian interactions [31].

For $h=0$ the RS susceptibility reduces to $\chi_{\mathrm{RS}}=\beta\left(\frac{1}{2}-q\right)$, according to (96), and thus obeys $\lim _{T \rightarrow 0} \chi_{\mathrm{RS}}=\sqrt{\pi} / 4 K$ and $\chi_{\mathrm{RS}}=1 / 2 T$ for $T \geq K$. Close to the critical point, expansion in $\tau=1-T / T_{c}$ reveals that $\chi_{\mathrm{RS}}=1 / 2 K+\mathcal{O}\left(\tau^{2}\right)$. $\chi_{\mathrm{RS}}$ thus increases from the value $\sqrt{\pi} / 4 K$ at $T=0$ to a cusp with value $1 / 2 K$ at $T=T_{c}$, followed by a monotonic $1 / 2 T$ decay to zero in the regime $T>T_{c}$ (see also figure 2 ).

Expression (94) for the AT instability of rotation invariant states simplifies similarly upon putting $h=0$ to

$$
\left[\frac{T}{K}\right]^{2}=\int_{0}^{\infty} d r r e^{-\frac{1}{2} r^{2}}\left\{\left[\frac{I_{2}[\Xi(r)]}{I_{0}[\Xi(r)]}-\frac{I_{1}^{2}[\Xi(r)]}{I_{0}^{2}[\Xi(r)]}\right]^{2}+\left[1-\frac{I_{1}^{2}[\Xi(r)]}{I_{0}^{2}[\Xi(r)]}\right]^{2}\right\}
$$

(with stability if the left-hand side is larger than the right-hand side). From this, in combination with the properties $I_{n>0}(0)=0$, we find upon inserting $q=0$ that the AT instability occurs at $T_{\mathrm{AT}}=K$, and thus coincides with the second order transition from a paramagnetic state to a $q>0$ state found earlier. In both models replica symmetry breaks as soon as we leave the paramagnetic region at $T=T_{c}=T_{\mathrm{AT}}=K$. Using the properties $I_{n}(z) / I_{0}(z)=1-n^{2} / 2 z+\mathcal{O}\left(z^{-2}\right)(|z| \rightarrow \infty)$ of the modified Bessel 
functions [30] we can also study the behaviour of both sides of (115) for $T \rightarrow 0$. This reveals that the degree of RS instability diverges near $T=0$ :

$$
\lim _{T \rightarrow 0} \frac{\mathrm{RHS}}{\mathrm{LHS}}=\int_{0}^{\infty} \frac{d r}{r} e^{-\frac{1}{2} r^{2}}=\infty
$$

Finally we work out the functions 104, 105) who's zeros mark continuous transitions away from the rotation-invariant state. For $h=0$ it follows from (112) that the two types of symmetry breaking coincide, i.e. $\Sigma_{\mathrm{II}}^{\text {rot }}=\Sigma_{\mathrm{LI}}^{\text {ref }}=\Sigma_{\mathrm{I}, \mathrm{II}}$. For $h \rightarrow 0$ all integrals over $\psi$ become trivial, and the quantities (106, 107, 108) reduce to

$$
\begin{aligned}
& \rho_{1}=\frac{1}{4} \int_{0}^{\infty} d r r e^{-\frac{1}{2} r^{2}}\left\{1-\frac{I_{2}^{2}[\Xi(r)]}{I_{0}^{2}[\Xi(r)]}\right\} \\
& \rho_{2}=\frac{1}{2} \int_{0}^{\infty} d r r e^{-\frac{1}{2} r^{2}}\left\{1-\frac{I_{1}^{2}[\Xi(r)]}{I_{0}^{2}[\Xi(r)]}\right\}\left\{1-\frac{3 I_{1}^{2}[\Xi(r)]}{I_{0}^{2}[\Xi(r)]}\right\} \\
& \rho_{3}=\int_{0}^{\infty} d r r e^{-\frac{1}{2} r^{2}}\left\{1-\frac{I_{2}[\Xi(r)]}{I_{0}[\Xi(r)]}\right\} \frac{I_{1}^{2}[\Xi(r)]}{I_{0}^{2}[\Xi(r)]}
\end{aligned}
$$

For $T \geq T_{c}=K$, where $\Xi(r)=0$, one simply obtains $\left(\rho_{1}, \rho_{2}, \rho_{3}\right)=\left(\frac{1}{4}, \frac{1}{2}, 0\right)$. Hence

$$
T \geq K: \quad \Sigma_{\mathrm{I}}(T)=(T / K)^{2}-1 \quad \Sigma_{\mathrm{II}}(T)=(T / K)^{2}+\frac{1}{2}
$$

Close to the critical point, expansion in $\tau=1-T / K$ reveals that $\left(\rho_{1}, \rho_{2}, \rho_{3}\right)=$ $\left(\frac{1}{4}, \frac{1}{2}-2 \tau, \tau\right)+\mathcal{O}\left(\tau^{3 / 2}\right)$, so

$$
\tau=1-T / K: \quad \Sigma_{\mathrm{I}}(T)=2 \tau+\mathcal{O}\left(\tau^{\frac{3}{2}}\right) \quad \Sigma_{\mathrm{II}}(T)=\frac{3}{2}-2 \tau+\mathcal{O}\left(\tau^{\frac{3}{2}}\right)
$$

Thus for $T>K$ the rotation-invariant RS solution is stable, with in the case of model I this stability becoming marginal at $T=T_{c}=K$, followed by restored stability for $T<T_{c}$. Close to $T=0$ one finds $\left(\rho_{1}, \rho_{2}, \rho_{3}\right)=(T \sqrt{\pi} / 2 K)(1,-1,2)+\mathcal{O}\left(T^{2}\right)$, so either $\Sigma_{\mathrm{I}, \mathrm{II}}(T)$ does not exist, or $\Sigma_{\mathrm{I}, \mathrm{II}}(T)=\mathcal{O}\left(T^{2}\right)$.

In figure 2 we show our theoretical results, together with those of homogeneously distributed pinning angles (to be studied next), by plotting the RS order parameter $q$ and the susceptibility $\chi_{\mathrm{RS}}$ as functions of temperature, and testing them against numerical simulations. We find reasonable agreement, given the CPU limitations on system size and equilibration times. In spite of the differences between models I and II at the microscopic level (notably in terms of frustration properties of spin loops), in the absence of pinning fields there is no macroscopic distinction between their physical behaviour in the replica-symmetric state, probably due to the overruling amount of frustration. The models have identical $q \neq 0$ and RSB transition lines and identical values of the replica-symmetric physical observables, with a paramagnetic state for $T>K$, and a spin-glass state for $T<K$. The only difference is the degree of stability of the rotation invariant state against non-rotationally-invariant fluctuations (since $\left.\Sigma_{\mathrm{I}}(T) \neq \Sigma_{\mathrm{II}}(T)\right)$, which cannot be measured directly.

\subsection{Homogeneously distributed pinning angles}

Our second choice is the homogeneous pinning angle distribution $p(\phi)=(2 \pi)^{-1}$. The Hamiltonian (2) is no longer invariant under simultaneous rotation of all spins, but we 

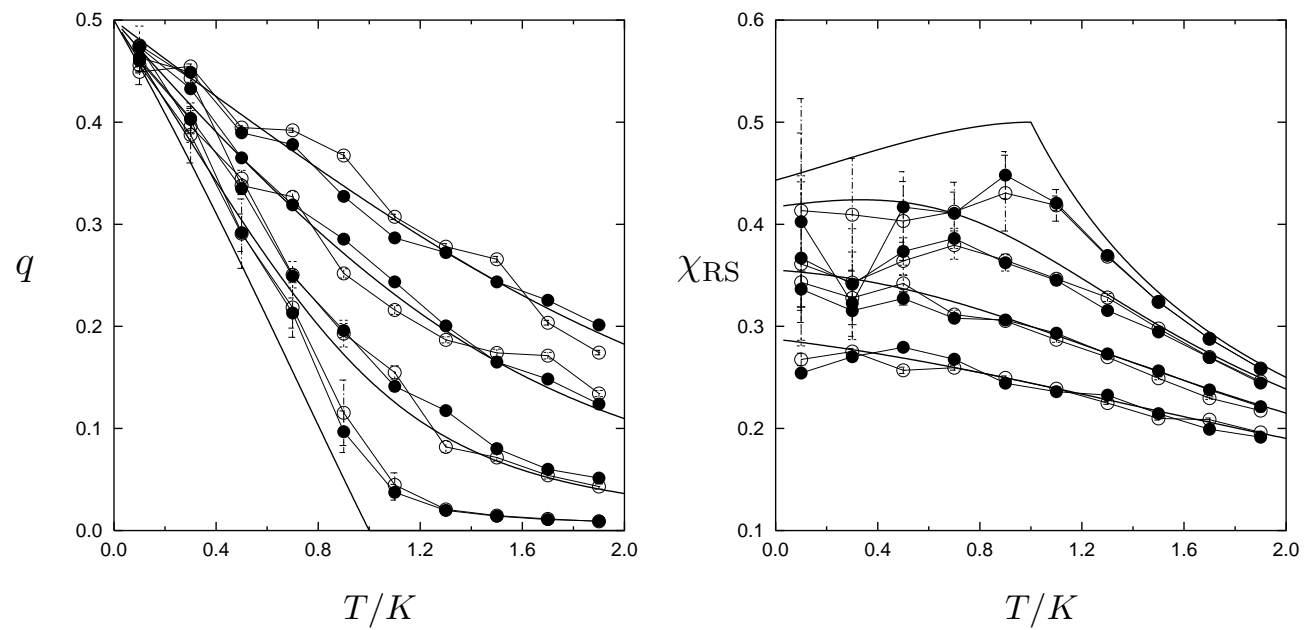

Figure 2. Theoretical predictions for the RS order parameter $q$ (left) and the susceptibility $\chi_{\mathrm{RS}}$ (right) as functions of temperature, for models I and II and with $K=1$, in the case of homogeneously distributed pinning angles. Different curves correspond to different values of the pinning field strength $h$, taken from $\{0,1,2,3\}$ (lower to upper in left picture, upper to lower in right picture). Connected markers: results of numerical simulations with $N=400$ (model I: $\bullet$, model II: o).

still expect the system globally to have full rotation symmetry. We cannot simplify equations $84,86,95$ ) further:

$$
\begin{gathered}
q=\int_{0}^{\pi} \frac{d \psi}{2 \pi} \int_{0}^{\infty} d r r e^{-\frac{1}{2} r^{2}}\left\{\frac{I_{1}[\Xi(r, \psi)]}{I_{0}[\Xi(r, \psi)]}\right\}^{2} \\
f[q]=-2 \beta K^{2}\left(q-\frac{1}{2}\right)^{2}-\frac{1}{\beta} \int_{0}^{\pi} \frac{d \psi}{\pi} \int_{0}^{\infty} d r r e^{-\frac{1}{2} r^{2}} \log I_{0}[\Xi(r, \psi)] \\
\frac{\chi_{\mathrm{RS}}}{\beta}=\frac{1}{2}-q-\int_{0}^{\pi} \frac{d \psi}{2 \pi} \int_{0}^{\infty} d r r e^{-\frac{1}{2} r^{2}}\left[\frac{I_{2}[\Xi(r, \psi)]}{I_{0}[\Xi(r, \psi)]}-\frac{I_{1}^{2}[\Xi(r, \psi)]}{I_{0}^{2}[\Xi(r, \psi)]}\right] \\
\times\left[\frac{h^{2}+4 K^{2} r^{2} q \cos (2 \psi)+4 h K r \sqrt{q} \cos (\psi)}{h^{2}+4 K^{2} r^{2} q+4 h K r \sqrt{q} \cos (\psi)}\right]
\end{gathered}
$$

with $\Xi(r, \psi)=\beta \sqrt{4 q K^{2} r^{2}+h^{2}+4 K h r \sqrt{q} \cos (\psi)}$. For $h \neq 0$ one no longer expects to find a phase transition from a $q=0$ to a $q>0$ state; this is clear upon expanding (119) for $\beta \rightarrow 0$, giving $q=\frac{1}{8}(\beta h)^{2}+\mathcal{O}\left(\beta^{3}\right)$. For weak fields one has $q=h^{2} / 8\left(T^{2}-K^{2}\right)+\mathcal{O}\left(h^{3}\right)$, in the regime $T>K$. Expansion for strong fields, $h \rightarrow \infty$, gives the leading orders

$$
q \rightarrow \frac{1}{2}, \quad \bar{f} \rightarrow-h, \quad \chi_{\mathrm{RS}} \rightarrow 0 \quad(h \rightarrow \infty)
$$

For $T \rightarrow 0$, on the other hand, one finds

$$
q=\frac{1}{2}-T \int_{0}^{\pi} \frac{d \psi}{2 \pi} \int_{0}^{\infty} \frac{d r r e^{-\frac{1}{2} r^{2}}}{\sqrt{h^{2}+2 K^{2} r^{2}+2 K h r \sqrt{2} \cos (\psi)}}+\mathcal{O}\left(T^{2}\right)
$$




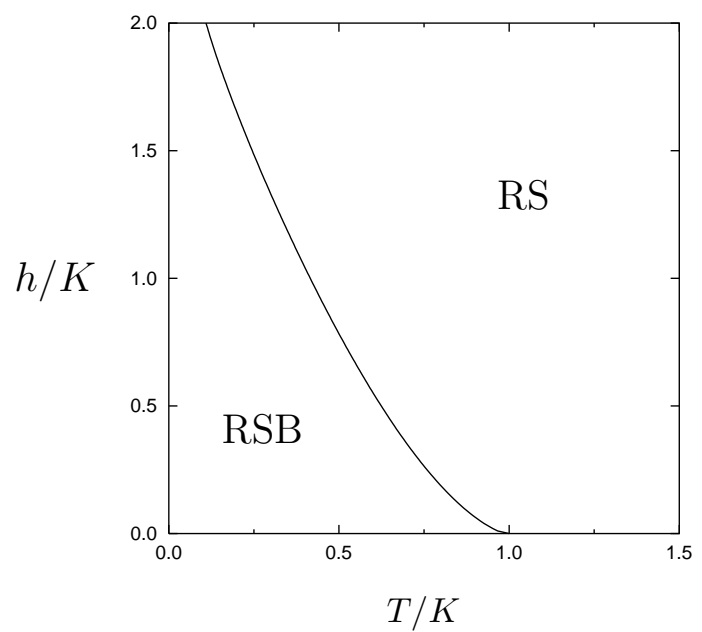

Figure 3. The phase diagram for models I and II, in the case of homogeneously distributed pinning angles. Solid: the AT instability, signaling a second order transition from a replica symmetric paramagnetic state (RS) to a locally ordered state without replica symmetry (RSB), and possibly to states without global spherical symmetry. The transition field strength diverges as $T / K \rightarrow 0$.

$$
\lim _{T \rightarrow 0} \chi_{\mathrm{RS}}=\int_{0}^{\pi} \frac{d \psi}{\pi} \int_{0}^{\infty} d r r e^{-\frac{1}{2} r^{2}} \frac{[h+\sqrt{2} K r \cos (\psi)]^{2}}{\left[h^{2}+2 K^{2} r^{2}+2 K h r \sqrt{2} \cos (\psi)\right]^{3 / 2}}
$$

All expression reduce for $h \rightarrow 0$ to those obtained in the previous sub-section (as they should). Away from $T, h \in\{0, \infty\}$ we must resort mainly to numerical evaluation of our equations. In figure 2 we compare the result of this exercise with numerical simulations, carried out for $N=400$ and $h \in\{0,1,2,3\}$. The agreement is satisfactory, apart from the $h=0$ and low temperature results, where finite size effects and equilibration problems are most prominent. We note, in comparison with the phase diagram of figure 3, that the the most serious deviations, observed in the susceptibility curves, occur in the RSB region where $\chi_{\mathrm{RS}}$ is indeed not expected to be correct.

Next we turn to our expression (124) for the AT line:

$$
\left[\frac{T}{K}\right]^{2}=\int_{0}^{\pi} \frac{d \psi}{\pi} \int_{0}^{\infty} d r r e^{-\frac{1}{2} r^{2}}\left\{\left[\frac{I_{2}[\Xi(r, \psi)]}{I_{0}[\Xi(r, \psi)]}-\frac{I_{1}^{2}[\Xi(r, \psi)]}{I_{0}^{2}[\Xi(r, \psi)]}\right]^{2}+\left[1-\frac{I_{1}^{2}[\Xi(r, \psi)]}{I_{0}^{2}[\Xi(r, \psi)]}\right]^{2}\right\}
$$

For $h \rightarrow 0$ we recover (115) with replica instability for $T<K$. Expanding (124) for weak fields in the regime $T>K$ gives $(T / K)^{2}=1-h^{2} / 2\left(T^{2}-K^{2}\right)+\mathcal{O}(h \sqrt{h})$, which has no solution; hence weak fields strengthen replica stability for $T>K$ (as expected). For $h \rightarrow \infty$ we find $\Xi(r, \psi) \rightarrow \beta h$, as a result of which we get, using $I_{n}(z) / I_{0}(z)=1-n^{2} / 2 z+\mathcal{O}\left(z^{-2}\right)(|z| \rightarrow \infty)$, for the right-hand side of (124) the asymptotic form $R H S=T^{2} / 2 h^{2}+\mathcal{O}\left(h^{-3}\right)(h \rightarrow \infty)$. Hence for every nonzero temperature there is a pinning field strength above which replica symmetry holds. Investigation of the limit $T \rightarrow 0$ in (124), upon again using the asymptotic forms of the modified Bessel functions inside integrals, we now end up with the divergent 
expression

$$
\begin{aligned}
\lim _{T \rightarrow 0} \frac{\mathrm{RHS}}{(T / K)^{2}} & =\int_{0}^{\pi} \frac{d \psi}{\pi} \int_{0}^{\infty} \frac{d r r e^{-\frac{1}{2} r^{2}}}{r^{2}+\frac{1}{2} h^{2} / K^{2}+\sqrt{2} r h \cos \psi / K} \\
& =\int \frac{D x D y}{(x+h / K \sqrt{2})^{2}+y^{2}}=e^{-\frac{1}{4} h^{2} / K^{2}} \int \frac{D x D y}{x^{2}+y^{2}} e^{x h / K \sqrt{2}} \\
& =e^{-\frac{1}{4} h^{2} / K^{2}} \int_{0}^{\infty} \frac{d r}{r} e^{-\frac{1}{2} r^{2}} I_{0}[h r / K \sqrt{2}]=\infty
\end{aligned}
$$

Thus the field strength $h_{\mathrm{AT}}(T)$ for which the AT instability occurs diverges as $T / K \rightarrow 0$. For general fields and temperatures the integrals in (124) will have to be evaluated numerically. This gives rise to the phase diagram shown in figure 3 .

For homogeneously distributed pinning angles, where $\langle\cos (4 \phi)\rangle_{\phi}=0$, it again follows from (112) that the two types of rotation symmetry breaking coincide: for all $T$ one has $\Sigma_{\mathrm{I}, \mathrm{II}}^{\mathrm{rot}}(T)=\Sigma_{\mathrm{I}, \mathrm{II}}^{\mathrm{ref}}(T)=\Sigma_{\mathrm{I}, \mathrm{II}}(T)$. The constituents $\left\{\rho_{1}, \rho_{2}, \rho_{3}\right\}$ of (104,105) become

$$
\begin{aligned}
& \rho_{1}=\frac{1}{4} \int_{0}^{\infty} d r r e^{-\frac{1}{2} r^{2}} \int_{0}^{\pi} \frac{d \psi}{\pi}\left\{1-\frac{I_{2}^{2}[\Xi(r, \psi)]}{I_{0}^{2}[\Xi(r, \psi)]}\right\} \\
& \rho_{2}=\frac{1}{2} \int_{0}^{\infty} d r r e^{-\frac{1}{2} r^{2}} \int_{0}^{\pi} \frac{d \psi}{\pi}\left\{1-\frac{I_{1}^{2}[\Xi(r, \psi)]}{I_{0}^{2}[\Xi(r, \psi)]}\right\}\left\{1-\frac{3 I_{1}^{2}[\Xi(r, \psi)]}{I_{0}^{2}[\Xi(r, \psi)]}\right\} \\
& \rho_{3}=\int_{0}^{\infty} d r r e^{-\frac{1}{2} r^{2}} \int_{0}^{\pi} \frac{d \psi}{\pi}\left\{1-\frac{I_{2}[\Xi(r, \psi)]}{I_{0}[\Xi(r, \psi)]}\right\} \frac{I_{1}^{2}[\Xi(r, \psi)]}{I_{0}^{2}[\Xi(r, \psi)]}
\end{aligned}
$$

Since $\left|I_{n}[z] / I_{0}[z]\right| \leq 1$ it is clear that $\rho_{1} \in\left[0, \frac{1}{4}\right], \rho_{2} \in\left[-1, \frac{1}{2}\right]$, and $\rho_{3} \in[0,1]$. Hence, given existence of the square root, one immediately obtains from 104, 105) the bounds $\Sigma_{\mathrm{I}}(T) \geq(T / K)^{2}-1$ and $\Sigma_{\mathrm{II}}(T) \geq(T / K)^{2}-\frac{3}{4}$. Thus the rotation invariant state is guaranteed to be stable for $T \geq K$ (model I) and $T>\frac{1}{2} \sqrt{3}$ (model II). For weak fields we may again, in the regime $T>K$, expand in powers of $h$. This gives

$$
\begin{aligned}
& \Sigma_{\mathrm{I}}(T)=\left[\frac{T}{K}\right]^{2}-1+\frac{h^{2}}{T^{2}-K^{2}}+\mathcal{O}\left(h^{3}\right) \\
& \Sigma_{\mathrm{II}}(T)=\left[\frac{T}{K}\right]^{2}+\frac{1}{2}+\mathcal{O}\left(h^{3}\right)
\end{aligned}
$$

In leading order, weak pinning fields increase the stability of the rotation-invariant state for model I, but do not effect the stability for model II. For strong pinning fields we expand our equations in powers of $h^{-1}$, which gives $\Sigma_{\mathrm{I}, \mathrm{II}}(T)=(T / K)^{2}+\mathcal{O}\left(h^{-2}\right)$, so that rotation symmetry is now stable for any temperature.

For arbitrary pinning field strengths and temperatures the integrals in (125, 126, 127) must be evaluated numerically. This reveals that for all non-zero temperatures and all field strengths $\Sigma_{\mathrm{I}, \mathrm{II}}(T) \geq 0$, implying (in combination with lacking evidence of first order transitions, and within the RS ansatz) the prediction that the system is always in a rotation-invariant state. Thus there are no further RS transitions, and the phase diagram is given by figure 3. As with absent pinning angles, models I and II differ only in the degree of stability of the rotation invariant state against non-rotationally-invariant fluctuations. 


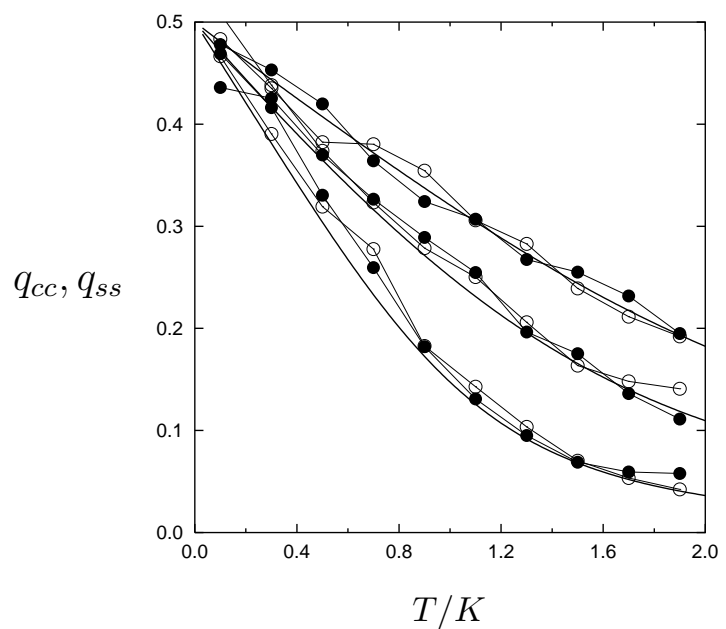

Figure 4. Theoretical predictions for the RS order parameters $q_{c c}$ and $q_{s s}$ as functions of temperature, for model I with $K=1$, in the case of pinning angle distribution $p(\phi)=\frac{1}{2} \delta\left[\phi-\frac{1}{4} \pi\right]+\frac{1}{2} \delta\left[\phi+\frac{1}{4} \pi\right]$ (where the theory predicts that $\left.q_{c c}=q_{s s}\right)$. Different curves correspond to different values of the pinning field strength $h$, taken from $\{1,2,3\}$ (lower to upper). Markers: results of numerical simulations with $N=400\left(q_{c c}: \bullet, q_{s s}:\right.$ o). Error bars in the measurements are of the order of 0.02 ; the deviations observed are mainly finite size effects. Note that the property $q_{c c}=q_{s s}$ is the fingerprint of rotation-invariant states.

\subsection{Inhomogeneous distributions: $p(\phi)=\frac{1}{2} \delta[\phi-\alpha]+\frac{1}{2} \delta[\phi+\alpha]$}

Finally we turn to pinning angle distributions of the form $p(\phi)=\frac{1}{2} \delta[\phi-\alpha]+\frac{1}{2} \delta[\phi+\alpha]$, with $\alpha \in[0, \pi]$. By varying the parameter $\alpha$ we can control this distribution to be either uni-modal or bi-modal. One now has $\langle\cos (2 \phi)\rangle_{\phi}=\cos (2 \alpha)$ and $\langle\cos (4 \phi)\rangle_{\phi}=$ $\cos (4 \alpha)$. Hence, according to (68) we can now have rotation-invariant solutions only when $\alpha \in\{\pi / 4,3 \pi / 4\}$; in the latter two cases one has $\langle\cos (4 \phi)\rangle_{\phi}=-1$, so as far as rotation-invariant solutions and their stability properties are concerned the $\alpha \in\{\pi / 4,3 \pi / 4\}$ models behave identically. For $\alpha \notin\{\pi / 4,3 \pi / 4\}$ there is generally only the overall reflection symmetry $\phi_{i} \rightarrow-\phi_{i}$ to be exploited, and we therefore must resort mainly to evaluating our equations for reflection symmetry states (where $\left.q_{c s}=Q_{c s}=0\right)(43,44,46)$, numerically.

In view of the above we concentrated on the cases $\alpha \in\{0, \pi / 4, \pi / 2\}$, which cover bi-modal $(\alpha=\pi / 4, \pi / 2)$ and uni-modal $(\alpha=0)$ distributions, and models with rotation invariant solutions $(\alpha=\pi / 4)$ as well as cases without $(\alpha=0, \pi / 2)$. We tested our theory by comparison with numerical simulations. The general effect of random pinning fields is to break the symmetry between models I and II, and the values of otherwise identical order parameter pairs such as $\left\{q_{c c}, q_{s s}\right\}$ and $\left\{Q_{c c}, Q_{s s}\right\}$. The agreement between theory and simulations is generally satisfactory, except for the susceptibility when measured at low values of $T$ and $h$, where one faces difficulties associated with equilibration and replica symmetry breaking. 
A: $\quad p(\phi)=\frac{1}{2} \delta\left[\phi-\frac{1}{4} \pi\right]+\frac{1}{2} \delta\left[\phi+\frac{1}{4} \pi\right]$

For $\alpha=\pi / 4$ one still has rotation-invariant solutions to our RS order parameter equations. In rotation invariant states all results obtained earlier for $p(\phi)=(2 \pi)^{-1}$ relating to the order parameter $q(119)$, the free energy per oscillator (120), the RS susceptibility $\chi_{\mathrm{RS}}(121)$ and the AT line $(124)$ can be taken over without alteration. The difference with $p(\phi)=(2 \pi)^{-1}$ is in the stability properties against fluctuations which violate rotational symmetry. Since here $\langle\cos (4 \pi)\rangle_{\phi}=-1$, we now obtain the symmetry breaking transitions as the zeros of the following functions (using (112)):

$$
\begin{aligned}
& \Sigma_{\mathrm{I}}^{\mathrm{rot}}(T)=(T / K)^{2}-\sqrt{\left(\rho_{1}(-1)-\rho_{2}(-1)\right)^{2}-\rho_{3}^{2}(-1)}-\rho_{1}(-1)-\rho_{2}(-1) \\
& \Sigma_{\mathrm{I}}^{\mathrm{ref}}(T)=(T / K)^{2}-\sqrt{\left(\rho_{1}(+1)-\rho_{2}(+1)\right)^{2}-\rho_{3}^{2}(+1)}-\rho_{1}(+1)-\rho_{2}(+1) \\
& \Sigma_{\mathrm{II}}^{\mathrm{rot}}(T)=(T / K)^{2}-\sqrt{\left(\rho_{1}(-1)-\rho_{2}(-1)\right)^{2}-\rho_{3}^{2}(-1)}+\rho_{1}(-1)+\rho_{2}(-1) \\
& \Sigma_{\mathrm{II}}^{\mathrm{ref}}(T)=(T / K)^{2}-\sqrt{\left(\rho_{1}(+1)-\rho_{2}(+1)\right)^{2}-\rho_{3}^{2}(+1)}+\rho_{1}(+1)+\rho_{2}(+1)
\end{aligned}
$$

with

$$
\begin{aligned}
& \rho_{1}(\kappa)=\frac{1}{4} \int_{0}^{\infty} d r r e^{-\frac{1}{2} r^{2}} \int_{0}^{\pi} \frac{d \psi}{\pi}\left\{1-\frac{I_{2}^{2}[\Xi(r, \psi)]}{I_{0}^{2}[\Xi(r, \psi)]}\right\} \\
& +\frac{1}{4} \kappa \int_{0}^{\infty} d r r e^{-\frac{1}{2} r^{2}} \int_{0}^{\pi} \frac{d \psi}{\pi}\left\{\frac{I_{4}[\Xi(r, \psi)]}{I_{0}[\Xi(r, \psi)]}-\frac{I_{2}^{2}[\Xi(r, \psi)]}{I_{0}^{2}[\Xi(r, \psi)]}\right\} \\
& \times\left\{1-\frac{8 \beta^{4}[2 K r \sqrt{q} \sin (\psi)]^{2}[h+2 K r \sqrt{q} \cos (\psi)]^{2}}{\Xi^{4}(r, \psi)}\right\} \\
& \rho_{2}(\kappa)=\frac{1}{2} \int_{0}^{\infty} d r r e^{-\frac{1}{2} r^{2}} \int_{0}^{\pi} \frac{d \psi}{\pi}\left\{1-\frac{I_{1}^{2}[\Xi(r, \psi)]}{I_{0}^{2}[\Xi(r, \psi)]}\right\}\left\{1-\frac{3 I_{1}^{2}[\Xi(r, \psi)]}{I_{0}^{2}[\Xi(r, \psi)]}\right\} \\
& +\frac{1}{2} \kappa \int_{0}^{\infty} d r r e^{-\frac{1}{2} r^{2}} \int_{0}^{\pi} \frac{d \psi}{\pi}\left\{\frac{I_{2}[\Xi(r, \psi)]}{I_{0}[\Xi(r, \psi)]}-\frac{I_{1}^{2}[\Xi(r, \psi)]}{I_{0}^{2}[\Xi(r, \psi)]}\right\}\left\{\frac{I_{2}[\Xi(r, \psi)]}{I_{0}[\Xi(r, \psi)]}-\frac{3 I_{1}^{2}[\Xi(r, \psi)]}{I_{0}^{2}[\Xi(r, \psi)]}\right\} \\
& \times\left\{1-\frac{8 \beta^{4}[2 K r \sqrt{q} \sin (\psi)]^{2}[h+2 K r \sqrt{q} \cos (\psi)]^{2}}{\Xi^{4}(r, \psi)}\right\} \\
& \rho_{3}(\kappa)=\int_{0}^{\infty} d r r e^{-\frac{1}{2} r^{2}} \int_{0}^{\pi} \frac{d \psi}{\pi}\left\{1-\frac{I_{2}[\Xi(r, \psi)]}{I_{0}[\Xi(r, \psi)]}\right\} \frac{I_{1}^{2}[\Xi(r, \psi)]}{I_{0}^{2}[\Xi(r, \psi)]} \\
& +\kappa \int_{0}^{\infty} d r r e^{-\frac{1}{2} r^{2}} \int_{0}^{\pi} \frac{d \psi}{\pi}\left\{1-\frac{I_{2}[\Xi(r, \psi)]}{I_{0}[\Xi(r, \psi)]}\right\}\left\{\frac{I_{1}^{2}[\Xi(r, \psi)]}{I_{0}^{2}[\Xi(r, \psi)]}-\frac{2 I_{2}[\Xi(r, \psi)]}{I_{0}[\Xi(r, \psi)]}\right\} \\
& \times\left\{1-\frac{8 \beta^{4}[2 K r \sqrt{q} \sin (\psi)]^{2}[h+2 K r \sqrt{q} \cos (\psi)]^{2}}{\Xi^{4}(r, \psi)}\right\}
\end{aligned}
$$

The inequality $\left|I_{n}[z] / I_{0}[z]\right| \leq 1$ allows one to obtain the crude general bound $T / K \leq 3$ for the zeros of (130,131,132,133). For strong pinning fields one has

$$
\left(\rho_{1}, \rho_{2}, \rho_{3}\right)=\frac{1-\kappa}{\beta h}(1,-1,2)+\mathcal{O}\left(h^{-2}\right) \quad(h \rightarrow \infty)
$$

and thus $\Sigma_{\mathrm{I}, \mathrm{II}}^{\mathrm{rot}, \mathrm{ref}}(T)=(T / K)^{2}+\mathcal{O}\left(h^{-2}\right)$, confirming stability of the rotation invariant state for sufficiently strong fields. For weak fields we may again in the regime $T>K$ expand in powers of $h$. This reveals that those terms in $\left\{\rho_{1}, \rho_{2}, \rho_{3}\right\}$ which are 
proportional to $\kappa$ are of sub-leading order $\mathcal{O}\left(h^{-3}\right)$ as $h \rightarrow 0$. From this it follows that we revert back to expressions (128, 129). Apparently, the leading order effect of switching on the pinning fields is again to stabilize the rotation invariant state.

For intermediate values of temperatures and fields we have evaluated (130,131, 132, 133) numerically, which showed that for all non-zero temperatures and all field strengths one has $\Sigma_{\mathrm{I}, \mathrm{II}}^{\text {rot,ref }}(T) \geq 0$. This, in turn, implies (in combination with lacking evidence of first order transitions, and within the RS ansatz) the non-trivial prediction that for $p(\phi)=\frac{1}{2} \delta\left[\phi-\frac{1}{4} \pi\right]+\frac{1}{2} \delta\left[\phi+\frac{1}{4} \pi\right]$, which is a pinning angle distribution without rotation invariance, the system is still always in a rotation-invariant state. This statement and its quantitative consequences are confirmed convincingly for model I by the numerical simulation data shown in 4 ; similar results can be shown for model II. We conclude that for $p(\phi)=\frac{1}{2} \delta\left[\phi-\frac{1}{4} \pi\right]+\frac{1}{2} \delta\left[\phi+\frac{1}{4} \pi\right]$ the phase diagram in the $(T / K, h / K)$ plane for both models I and II is simply identical to that of homogeneously distributed pinning angles, as shown in figure 3 .

$B: \quad p(\phi)=\delta[\phi]$

For $p(\phi)=\delta[\phi]$ we no longer have rotation invariant solutions, and the analysis becomes more complicated. Given the assumption of overall reflection symmetry $\left(Q_{c s}=q_{c s}=0\right.$, whose stability we will calculate below) we are left with three RS order parameters, $\left\{Q_{c c}, q_{c c}, q_{s s}\right\}$, and with the effective measures (43, 44 which now become

$$
\begin{aligned}
& M_{\mathrm{I}}(\theta \mid x, y)=e^{\beta h \cos (\theta)+(\beta K)^{2}\left[2 Q_{c c}-1+q_{s s}-q_{c c}\right] \cos (2 \theta)+2 \beta K\left[\cos (\theta) x \sqrt{q_{c c}}+\sin (\theta) y \sqrt{q_{s s}}\right]} \\
& M_{\mathbb{I}}(\theta \mid x, y)=e^{\beta h \cos (\theta)-(\beta K)^{2}\left[2 Q_{c c}-1+q_{s s}-q_{c c}\right] \cos (2 \theta)+2 \beta K\left[\cos (\theta) x \sqrt{q_{s s}}+\sin (\theta) y \sqrt{q_{c c}}\right]}
\end{aligned}
$$

The remaining order parameters are to be solved from the coupled equations

$$
Q_{c c}=\left\langle\left\langle\left\langle\left\langle\cos ^{2}(\theta)\right\rangle_{\star}\right\rangle\right\rangle\right\rangle_{\phi} \quad q_{c c}=\left\langle\left\langle\left\langle\langle\cos (\theta)\rangle_{\star}^{2}\right\rangle\right\rangle\right\rangle_{\phi} \quad q_{s s}=\left\langle\left\langle\left\langle\langle\sin (\theta)\rangle_{\star}^{2}\right\rangle\right\rangle\right\rangle_{\phi}
$$

At the RS ground state we know that $q_{c c}+q_{s s}=1$ and $Q_{c c}=\frac{1}{2}\left[1+q_{c c}-q_{s s}\right]$. Due to $p(\phi)=\delta[\phi]$ the RS susceptibility (55) simplifies immediately to:

$$
\chi_{\mathrm{RS}}=\beta\left(1-Q_{c c}-q_{s s}\right)
$$

For strong pinning fields one finds $\lim _{h \rightarrow \infty} Q_{c c}=\lim _{h \rightarrow \infty} q_{c c}=1$ and $\lim _{h \rightarrow \infty} q_{s s}=0$. Hence $\lim _{h \rightarrow \infty} \chi_{\mathrm{RS}}=0$. In the high temperature regime an expansion in powers of $\beta$ shows the solution of (139) to behave as

$$
Q_{c c}=\frac{1}{2}+\mathcal{O}\left(\beta^{2}\right), \quad q_{c c}=\frac{1}{4} \beta^{2} h^{2}+\mathcal{O}\left(\beta^{3}\right), \quad q_{s s}=\mathcal{O}\left(\beta^{3}\right)
$$

Hence $\chi_{\mathrm{RS}}=1 / 2 T+\mathcal{O}\left(T^{-3}\right)$ as $T \rightarrow \infty$.

The expressions for the AT line(s) (53,54) and reflection symmetry transitions (63,64) cannot be simplified further, except in special limits and in the special case described below. For $h \rightarrow \infty$ one trivially extracts from these equations that both replica symmetry and reflection symmetry are stable for all finite temperatures; the same is true for high temperatures and arbitrary field strengths (as expected).

However, analytical progress can be made for model I. Here one observes, in line with (141), that the order parameter equations allow for solutions with $q_{s s}=0$. Given the identification $q_{s s}=\lim _{N \rightarrow \infty} N^{-1} \sum_{i} \overline{\left\langle\sin \left(\theta_{i}\right)\right\rangle^{2}}$ such solutions imply that 

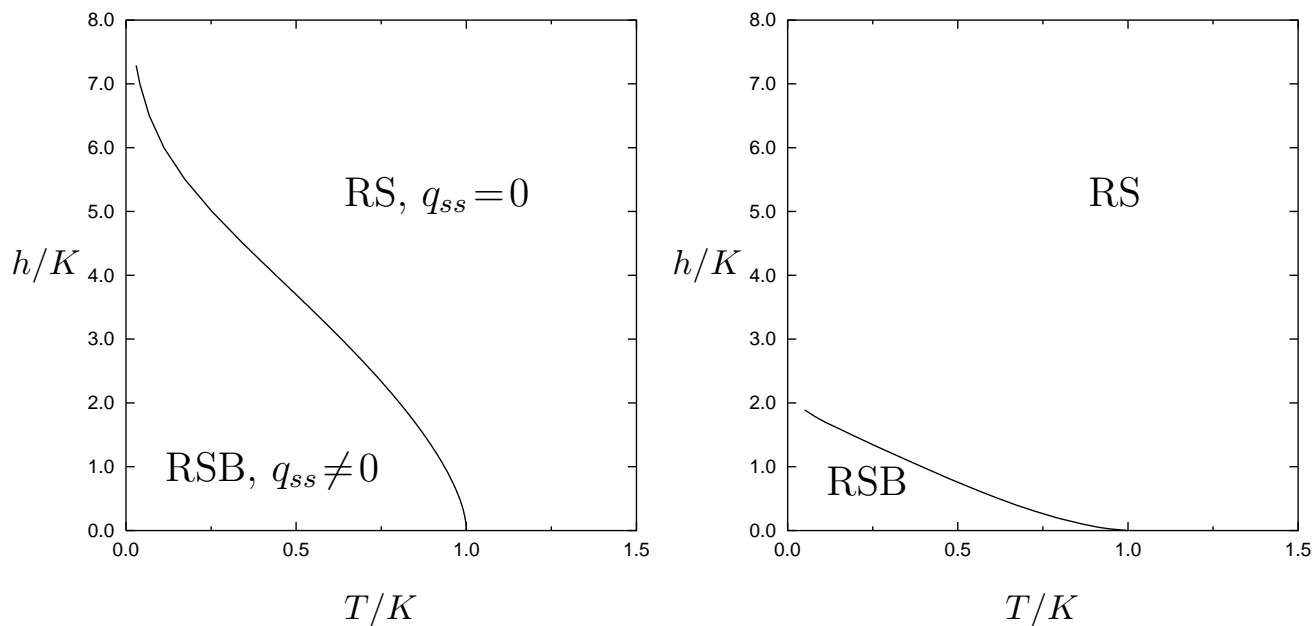

Figure 5. The phase diagrams for models I (left) and II (right), in the case of $p(\phi)=\delta[\phi]$. Solid: the AT instability, signaling a second order transition from a replica symmetric state (RS) to a state without replica symmetry (RSB). For model I the AT line coincides with line marking a continuous bifurcation of $q_{s s} \neq 0$ solutions of the RS order parameter equations.

$\left\langle\sin \left(\theta_{i}\right)\right\rangle=0$ for each oscillator. This can be understood as a result of the action of the pinning fields, which for $p(\phi)=\delta(\phi)$ tend to drive the oscillator phases towards $\phi_{i}=0$. Insertion of $q_{s s}=0$ as an ansatz into (1137) gives $\langle\sin (\theta)\rangle_{\star}=\langle\sin (\theta) \cos (\theta)\rangle_{\star}=0$, confirming $q_{s s}=0$ self-consistently (this is not possible for model II). This results in considerable analytical simplifications, such as $\gamma_{c s}=0$ and $\gamma_{s s}=\left\langle\sin ^{2}(\theta)\right\rangle_{\star}$, and also reduces the computational effort in the various numerical integrations. One now has only two RS order parameters, $Q_{c c}$ and $q_{c c}$, to be solved from

$$
Q_{c c}=\int D x\left\langle\cos ^{2}(\theta)\right\rangle_{\star}, \quad q_{c c}=\int D x\langle\cos (\theta)\rangle_{\star}^{2}
$$

with the simplified effective measure

$$
M_{\mathrm{I}}(\theta \mid x)=e^{\beta \cos (\theta)\left[h+2 K x \sqrt{q_{c c}}\right]+(\beta K)^{2}\left[2 Q_{c c}-1-q_{c c}\right] \cos (2 \theta)}
$$

The ground state has $Q_{c c}=q_{c c}=1$. The equation for the AT line of model I becomes

$$
(T / 2 K)^{2}=\max \left\{\int D x\left[\left\langle\cos ^{2}(\theta)\right\rangle_{\star}-\langle\cos (\theta)\rangle_{\star}^{2}\right]^{2}, \int D x\left\langle\sin ^{2}(\theta)\right\rangle_{\star}^{2}\right\}
$$

Similarly we can for $q_{s s}=0$ simplify the constituents $\left\{\lambda_{i}\right\}$ of (63) to

$$
\begin{aligned}
& \lambda_{1}=2 \int D x\left\langle\sin ^{2}(\theta) \cos ^{2}(\theta)\right\rangle_{\star} \\
& \lambda_{2}=2 \int D x\left\langle\sin ^{2}(\theta)\right\rangle_{\star}\left[\left\langle\cos ^{2}(\theta)\right\rangle_{\star}-2\langle\cos (\theta)\rangle_{\star}^{2}\right] \\
& \lambda_{3}=4 \int D x\left\langle\sin ^{2}(\theta) \cos (\theta)\right\rangle_{\star}\langle\cos (\theta)\rangle_{\star}
\end{aligned}
$$


These are to be inserted into (63), whose zeros mark reflection symmetry breaking. Finally we now have a third type of transition: de-stabilization of $q_{s s}=0$. The condition for this, $\partial^{2} \bar{f}_{I}[\ldots] / \partial q_{s s}^{2}=0$, can with help of Appendix A be written as

$$
(T / 2 K)^{2}=\int D x\left\langle\sin ^{2}(\theta)\right\rangle_{\star}^{2}
$$

Clearly, for $h \rightarrow 0$ (absent pinning fields, as studied in the first part of this section) the $q_{s s} \neq 0$ bifurcation occurs at $T=K$, and coincides with a bifurcation of $q_{c c} \neq 0$ and with the AT line. Comparison with (144) shows that the critical temperature $T_{c}$ defined by (148) obeys $T_{c} \leq T_{\mathrm{AT}}$. Hence the AT instability does occur for $q_{s s}=0$ and is thus given by (144). Numerical analysis reveals that along the line (144) one always has $\int D x\left[\left\langle\cos ^{2}(\theta)\right\rangle_{\star}-\langle\cos (\theta)\rangle_{\star}^{2}\right]^{2} \leq \int D x\left\langle\sin ^{2}(\theta)\right\rangle_{\star}^{2}$, and hence $T_{A T}=T_{c}$ (the two transition lines coincide, in agreement with [26, 27]).

Numerical solution of equations $(53,54)$ and (148) lead us to the phase diagrams shown in figure 5. The $q_{s s} \neq 0$ transition for model I coincides with the AT line. We observe that the RS stabilizing effect of the pinning fields is much greater for model II than for model I, as reflected in a significantly lower RSB transition temperature for any nonzero field strength. Numerical evaluation of the conditions (63.64) marking the continuous breaking of reflection symmetry shows for both models that this does not happen at any finite temperature; reflection symmetry remains locally stable.

The results of comparing the solutions of our RS order parameter equations (obtained numerically) with simulations are shown in figures 6 (model I) and 7 (model II). It is clear from the figures 6 and 7 that, due to the breaking of global rotation symmetry by the external pinning fields, there is a now a non-trivial difference between the macroscopic behaviour of our two models. The energetic conflicts in the two models are resolved differently. With the exception of the susceptibility, the agreement between theory and numerical experiment is good, taking into account finite size effects which lead as usual to a smoothening of the second order phase transition marking $q_{s s} \neq 0$ bifurcations. Comparison with the locations of the AT lines in figure 5 suggests again that the serious deviations in the susceptibility are due to replica symmetry breaking; $\chi_{\mathrm{RS}}$ cannot be expected to be correct in the RSB region.

$C: \quad p(\phi)=\frac{1}{2} \delta\left[\phi-\frac{1}{2} \pi\right]+\frac{1}{2} \delta\left[\phi+\frac{1}{2} \pi\right]$

Our final example is a bi-modal pinning angle distribution which, due to $\langle\cos (2 \theta)\rangle_{\phi}=$ $-1 \neq 0$, again does not allow for rotation invariant states. According to transformation (6) (with $\eta=\lambda=0$ ) this choice is related to the case $p(\phi)=\delta[\phi]$ by a simple gauge transformation, and our models must have identical free energies and phase diagrams. As a consistency test we will try to extract this property from the RS saddle-point equations. Given reflection symmetry, the two effective measures (43,44) now become

$$
\begin{aligned}
& M_{\mathrm{I}}\left(\theta \mid x, y, \pm \frac{\pi}{2}\right)=e^{ \pm \beta h \sin (\theta)+(\beta K)^{2}\left[2 Q_{c c}-1+q_{s s}-q_{c c}\right] \cos (2 \theta)+2 \beta K\left[\cos (\theta) x \sqrt{q_{c c}}+\sin (\theta) y \sqrt{q_{s s}}\right]} \\
& M_{\mathrm{II}}\left(\theta \mid x, y, \pm \frac{\pi}{2}\right)=e^{ \pm \beta h \sin (\theta)-(\beta K)^{2}\left[2 Q_{c c}-1+q_{s s}-q_{c c}\right] \cos (2 \theta)+2 \beta K\left[\cos (\theta) x \sqrt{q_{s s}}+\sin (\theta) y \sqrt{q_{c c}}\right]}
\end{aligned}
$$

We observe that our models can indeed be mapped onto those obtained for $p(\phi)=\delta[\phi]$. We introduce the transformation $\theta= \pm\left(\frac{1}{2} \pi-\theta^{\prime}\right)$ (permutation and reflection of 

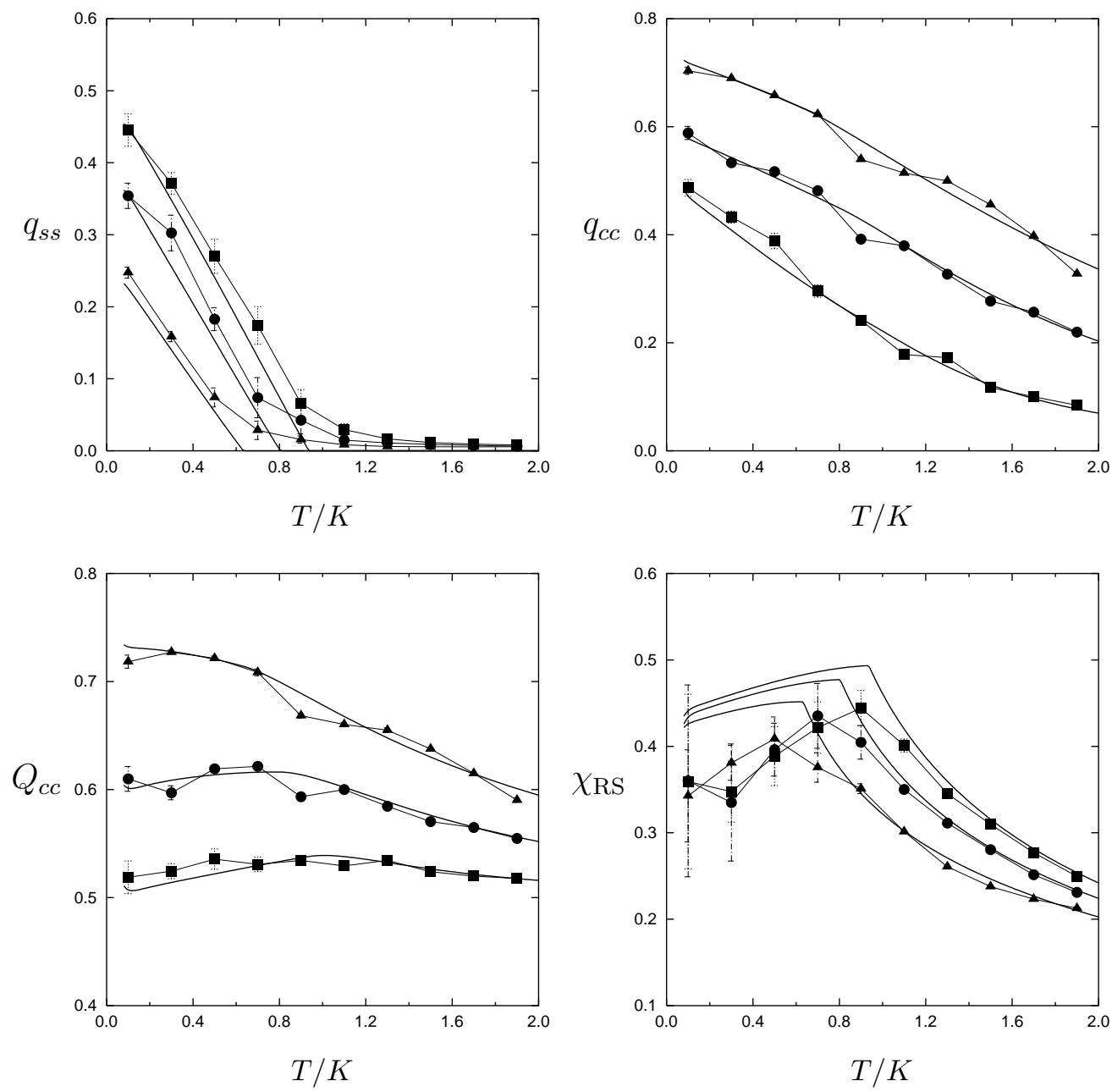

Figure 6. The theoretical predictions for the RS order parameters $\left\{q_{s s}, q_{c c}, Q_{c c}\right\}$ and the RS susceptibility $\chi_{\mathrm{RS}}$ as functions of temperature, for model I with $K=1$, in the case of $p(\phi)=\delta[\phi]$. Thick solid lines: theoretical predictions. Markers: results of numerical simulations with $N=800$. Different curves correspond to different values of the pinning field strength $h$, taken from $\{1,2,3\}$ (indicated by squares, circles and triangles, respectively).

axes), which, via the order parameter equations (139), induces a corresponding transformation of the order parameters:

$$
Q_{c c}=1-Q_{c c}^{\prime}, \quad q_{c c}=q_{s s}^{\prime}, \quad q_{s s}=q_{c c}^{\prime}
$$

The effect on the measures 1149,150 ) is that the latter can now be expressed in terms of the measures (137,138) describing the distribution $p(\phi)=\delta[\phi]$ studied previously:

$$
M_{\mathrm{I}}\left(\theta \mid x, y, \pm \frac{\pi}{2}\right)=M_{\mathrm{I}}\left(\theta^{\prime} \mid \pm y, x\right) \quad M_{\mathrm{II}}\left(\theta \mid x, y, \pm \frac{\pi}{2}\right)=M_{\mathrm{II}}\left(\theta^{\prime} \mid \pm y, x\right)
$$

Since operations such as $(x, y) \rightarrow( \pm y, x)$ have no physical consequences, and since all observables and transition lines are (within RS) constructed from the measures 

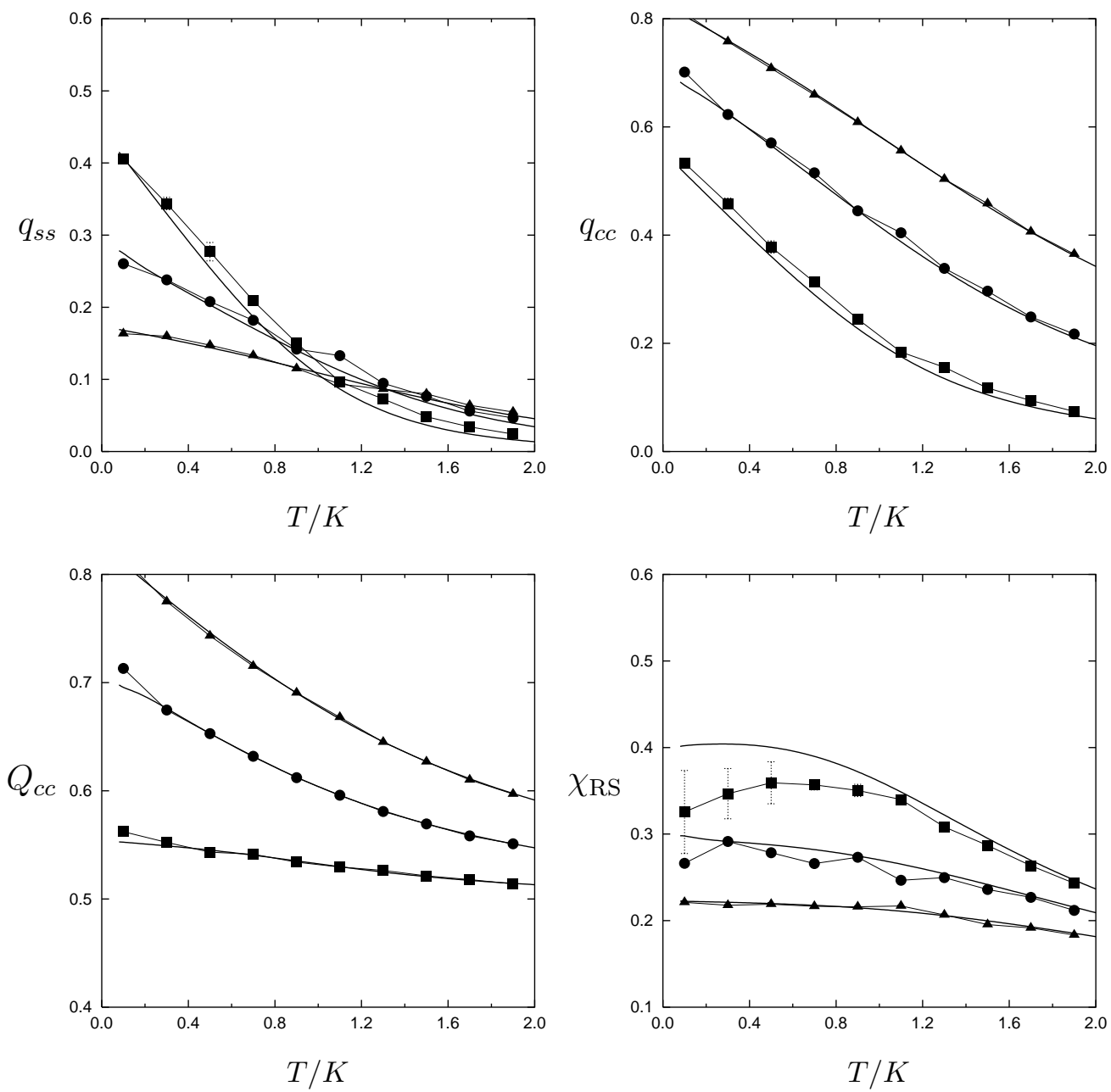

Figure 7. The theoretical predictions for the RS order parameters $\left\{q_{s s}, q_{c c}, Q_{c c}\right\}$ and the RS susceptibility $\chi_{\mathrm{RS}}$ as functions of temperature, for model II with $K=1$, in the case of $p(\phi)=\delta[\phi]$. Thick solid lines: theoretical predictions. Markers: results of numerical simulations with $N=800$. Different curves correspond to different values of the pinning field strength $h$, taken from $\{1,2,3\}$ (indicated by squares, circles and triangles, respectively).

(149, 150), we conclude that the macroscopic physics of the cases $p(\phi)=\delta[\phi]$ and $p(\phi)=\frac{1}{2} \delta\left[\phi-\frac{1}{2} \pi\right]+\frac{1}{2} \delta\left[\phi+\frac{1}{2} \pi\right]$ are indeed identical. The relations (151) map the order parameters $\left\{Q_{c c}, q_{c c}, q_{s s}\right\}$ of the present bi-modal distribution to the parameters $\left\{Q_{c c}^{\prime}, q_{c c}^{\prime}, q_{s s}^{\prime}\right\}$ of the case $p(\phi)=\delta[\phi]$, and both cases have identical free energies for all values of $\{T / K, h / K\}$ and identical phase diagrams. In particular, the $q_{s s}=0$ solution of model I for $p(\phi)=\delta[\phi]$ corresponds here to a solution with $q_{c c}=0$ (again for model I only), and the RS susceptibility now becomes $\chi_{\mathrm{RS}}=\beta\left(Q_{c c}-q_{c c}\right)$. The equivalence of the $p(\phi)=\delta[\phi]$ and $p(\phi)=\frac{1}{2} \delta\left[\phi-\frac{1}{2} \pi\right]+\frac{1}{2} \delta\left[\phi+\frac{1}{2} \pi\right]$ models is also immediately obvious in numerical simulations (which we do not show for brevity). 


\section{Discussion}

We have studied models of frustrated coupled Kuramoto oscillators in the presence of random pinning fields. To simplify our problem we have assumed that the natural frequency of all oscillators are the same and that the pinning fields distribution is reflection-symmetric, i.e. $p(\phi)=p(-\phi)$ (a simple gauge transform allows one to map the present models also to those with larger families of pinning field distributions). We have calculated the disorder-averaged free energy using the replica method for two types of random pair interactions (model I and model II, the first we standard ferroand anti-ferromagnetic interactions, the second with chiral interactions), which differ in the level of frustration. In terms of e.g. oscillator triplets, model I is partially bondfrustrated, whereas model II is fully bond-frustrated. For small system sizes the two models have significantly different ground state properties. Our main interest here is to understand the difference(s) in physical behaviour between the two models in equilibrium, in the thermodynamic limit. At the mathematical level our two models differ in the details of the effective (replicated) single-spin measure, but they have the same types of order parameters. Within the replica symmetric ansatz, one finds different expressions for the AT instabilities; more unexpectedly, the two models are also found to differ in the nature of the extremum of the free energy.

We inspected the effects of different types of symmetries which our models could inherit from the pinning field distribution. Global reflection symmetry leads to a simplified measure and a reduced number of order parameters. Reflection symmetry breaking transitions are found to be possible at most in the RSB regime. In the case of global rotation symmetry, only one order parameter remains (within RS), and the effective measure, the free enrgy, and the AT lines are identical for both models. We analyzed our order parameter equations in detail for four specific choices for the pinning fields, and tested our theoretical predictions against computer simulations. Without pinning fields, our two models both behave identically to a standard longrange XY model with non-chiral Gaussian interactions [31]. The same is found to be true for uniformly distributed pinning angles. The agreement between theory and simulation is quite satisfactory, modulo finite size effects, except for the susceptibility at low temperatures, where RSB effects in combination with equilibration difficulties play a major role. We then studied inhomogeneous pinning angle distributions, of the form $p(\phi)=\frac{1}{2} \delta[\phi-\alpha]+\frac{1}{2} \delta[\phi+\alpha]$. For the special value $\alpha=\frac{\pi}{4}$ one finds, remarkably, that our models are again always in a rotation invariant state (in spite of the fact that the pinning field distribution does not have rotation invariance); this is confirmed by simulations. For $\alpha=0$ the symmetry between our two models is broken. They now differ significantly in terms of the location of the AT line, which for model I in addition coincides with a further order parameter bifurcation, as in [26, 27]. The $\alpha 0 \pi 2$ case can be mapped onto the $\alpha=0$ one, by a suitable gauge transformation. Again, there is a good agreement between theory and simulations.

As a next stage it would be interesting to explore the behavior of these models for oscillators with random distributions of natural frequencies, as well as in the presence of more general chiral interactions. In both extensions/generalizations the standard equilibrium replica formalism can no longer be used; in the first case one has to rely on dynamical formalisms [5, 6], whereas in the second case one has to call upon renormalization tricks 20 . 
Acknowledgements The authors are grateful for support from the British Council (UK) and the Mcyt (grant BSM2000-0696) (Spain), and from the European Science Foundation (under the SPHINX programme).

\section{References}

[1] Winfree AT 19671980 The geometry of biological time Springer-Verlag, New York.

[2] Kuramoto Y 1984 Chemical oscillations, waves and turbulence Springer-Verlag, Berlin.

[3] Kuramoto Y 1984 Prog. Theor. Phys. Suppl 79223

[4] Strogatz SH and Mirollo RE 1991 J. Stat. Phys. 63613

[5] Bonilla LL, Neu JC and Spigler R 1992 J. Stat. Phys. 67313

[6] Bonilla LL, Perez Vicente CJ and Spigler R 1998 Physica D 11379

[7] Acebron JA, Bonilla LL, De Leo S and Spigler R 1998 Phys. Rev. E 575287

[8] Daido H 1988 Phys. Rev. Lett. 61231

[9] Strogatz SH and Mirollo RE 1988 J. Phys. A 21 L699

[10] Bonilla LL, Perez Vicente CJ and Rubi JM 1993 J. Stat. Phys. 70921

[11] Arenas A and Perez Vicente CJ 1994 Europhys. Lett. 2679

[12] Sakaguchi H 1988 Prog. Theor. Phys. 7939

[13] Reimann P, Van den Broeck C and Kawai R 1999 Phys. Rev. E 606402

[14] Schuster HG and Wagner P 1990 Biol. Cybern. 64 77, Biol. Cybern. 6483

[15] Abbott LF 1990 J. Phys. A 233835

[16] Wiesenfeld K, Colet P and Strogatz SH 1996 Phys. Rev. Lett. 76 404; 1998 Phys. Rev E 57 1563

[17] Granato E, Kosterlitz JM and Nightingale MP 1996 Physica B 222266

[18] Majhofer A 1996 Physica B 222273

[19] Sakaguchi H and Kuramoto Y 1986 Prog. Theor. Phys. 76576

[20] Rae HC, Coolen ACC and Sollich P 2002 in preparation

[21] Daido H 1992 Phys Rev Lett 681073 ,2000 Phys. Rev. E 612145

[22] Stiller JC and Radons G 1998 Phys. Rev. E 581789

[23] Choi MY and Doniach S 1985 Phys. Rev. B 314516

[24] Arenas A and Perez Vicente CJ 1994 Phys. Rev. E 50949

[25] Elderfield DJ and Sherrington D 1982 J. Phys. A 15 L513

[26] Cragg DM, Sherrington D and Gabay M Phys. Rev. Lett. 491982

[27] Elderfield D and Sherrington D 1983 J. Phys. C 164865

[28] De Almeida JRL and Thouless DJ 1978 J. Phys. A 11983

[29] Mézard M, Parisi G and Virasoro MA 1987 Spin-Glass Theory and Beyond (Singapore: World Scientific)

[30] Abramowitz M and Stegun IA 1972 Handbook of Mathematical Functions (New York: Dover)

[31] Kirkpatrick S and Sherrington D 1978 Phys. Rev. B 174384 


\section{Appendix A. Derivatives of the RS free energy}

The (first and second order) derivatives of $f[\ldots]$ in (30) with respect to the RS order parameters are calculated by working out the following general relations (where $\gamma$ and $\gamma^{\prime}$ denote any two order parameters from the set $\left.\left\{Q_{\star \star}, q_{\star \star}\right\}\right)$ :

$$
\begin{aligned}
\frac{1}{\beta K^{2}} \frac{\partial f}{\partial \gamma} & =\frac{\partial U}{\partial \gamma}-\frac{1}{\beta^{2} K^{2}}\left\langle\left\langle\left\langle\left\langle\frac{\partial \log M(\ldots)}{\partial \gamma}\right\rangle_{\star}\right\rangle\right\rangle\right\rangle_{\phi} \\
\frac{1}{\beta K^{2}} \frac{\partial^{2} f}{\partial \gamma \partial \gamma^{\prime}} & =\frac{\partial^{2} U}{\partial \gamma \partial \gamma^{\prime}}-\frac{1}{\beta^{2} K^{2}}\left\langle\left\langle\left\langle\left\langle\frac{\partial^{2} \log M(\ldots)}{\partial \gamma \partial \gamma^{\prime}}\right\rangle_{\star}\right.\right.\right. \\
+ & \left.\left.\left.\left.\left\langle\frac{\partial \log M(\ldots)}{\partial \gamma} \frac{\partial \log M(\ldots)}{\partial \gamma^{\prime}}\right\rangle_{\star}-\left\langle\frac{\partial \log M(\ldots)}{\partial \gamma}\right\rangle_{\star}\left\langle\frac{\partial \log M(\ldots)}{\partial \gamma^{\prime}}\right]\right\rangle_{\star}\right\rangle\right\rangle\right\rangle_{\phi}
\end{aligned}
$$

The Gaussian disorder variables $\{x, y, u, v\}$ generated by the differentiations are eliminated via integration by parts. Below we give the final results of the calculations, which, although not fundamentally complicated, can be lengthy.

Appendix A.1. First order derivatives

For model I one finds the following first order derivatives:

$$
\begin{aligned}
& \frac{1}{\beta K^{2}} \frac{\partial f_{\mathrm{I}}}{\partial Q_{c c}}=4\left\{Q_{c c}-\left\langle\left\langle\left\langle\left\langle\cos ^{2}(\theta)\right\rangle_{\star}\right\rangle\right\rangle\right\rangle_{\phi}\right\} \\
& \frac{1}{\beta K^{2}} \frac{\partial f_{\mathrm{I}}}{\partial Q_{c s}}=4\left\{Q_{c s}-\left\langle\left\langle\left\langle\langle\sin (\theta) \cos (\theta)\rangle_{\star}\right\rangle\right\rangle\right\rangle_{\phi}\right\} \\
& \frac{1}{\beta K^{2}} \frac{\partial f_{\mathrm{I}}}{\partial q_{c c}}=2\left\{\left\langle\left\langle\left\langle\langle\cos (\theta)\rangle_{\star}^{2}\right\rangle\right\rangle\right\rangle_{\phi}-q_{c c}\right\} \\
& \frac{1}{\beta K^{2}} \frac{\partial f_{\mathrm{I}}}{\partial q_{s s}}=2\left\{\left\langle\left\langle\left\langle\langle\sin (\theta)\rangle_{\star}^{2}\right\rangle\right\rangle\right\rangle_{\phi}-q_{s s}\right\} \\
& \frac{1}{\beta K^{2}} \frac{\partial f_{\mathrm{I}}}{\partial q_{c s}}=4\left\{\left\langle\left\langle\left\langle\langle\cos (\theta)\rangle_{\star}\langle\sin (\theta)\rangle_{\star}\right\rangle\right\rangle_{\phi}-q_{c s}\right\}\right.
\end{aligned}
$$

For model II one finds:

$$
\begin{aligned}
& \frac{1}{\beta K^{2}} \frac{\partial f_{\mathrm{II}}}{\partial Q_{c c}}=-4\left\{Q_{c c}-\left\langle\left\langle\left\langle\left\langle\cos ^{2}(\theta)\right\rangle_{\star}\right\rangle\right\rangle\right\rangle_{\phi}\right\} \\
& \frac{1}{\beta K^{2}} \frac{\partial f_{\mathrm{II}}}{\partial Q_{c s}}=-4\left\{Q_{c s}-\left\langle\left\langle\left\langle\langle\sin (\theta) \cos (\theta)\rangle_{\star}\right\rangle\right\rangle\right\rangle_{\phi}\right\} \\
& \frac{1}{\beta K^{2}} \frac{\partial f_{\mathrm{II}}}{\partial q_{c c}}=2\left\{\left\langle\left\langle\left\langle\langle\sin (\theta)\rangle_{\star}^{2}\right\rangle\right\rangle\right\rangle_{\phi}-q_{s s}\right\} \\
& \frac{1}{\beta K^{2}} \frac{\partial f_{\mathrm{II}}}{\partial q_{s s}}=2\left\{\left\langle\left\langle\left\langle\langle\cos (\theta)\rangle_{\star}^{2}\right\rangle\right\rangle\right\rangle_{\phi}-q_{c c}\right\} \\
& \frac{1}{\beta K^{2}} \frac{\partial f_{\mathrm{II}}}{\partial q_{c s}}=-4\left\{\left\langle\left\langle\left\langle\langle\cos (\theta)\rangle_{\star}\langle\sin (\theta)\rangle_{\star}\right\rangle\right\rangle\right\rangle_{\phi}-q_{c s}\right\}
\end{aligned}
$$

The above results re-confirm our replica-symmetric saddle-point equations.

Appendix A.2. Second order derivatives for model I

$\frac{1}{\beta K^{2}} \frac{\partial^{2} f_{\mathrm{I}}}{\partial Q_{c c}^{2}} \quad=4-4(\beta K)^{2}\left\langle\left\langle\left\langle\left\langle\cos ^{2}(2 \theta)\right\rangle_{\star}-\langle\cos (2 \theta)\rangle_{\star}^{2}\right\rangle\right\rangle\right\rangle_{\phi}$ 
Frustrated coupled oscillators with random fields - October 23rd, 2002

$$
\begin{aligned}
& \frac{1}{\beta K^{2}} \frac{\partial^{2} f_{\mathrm{I}}}{\partial Q_{c s}^{2}} \quad=4-4(\beta K)^{2}\left\langle\left\langle\left\langle\left\langle\sin ^{2}(2 \theta)\right\rangle_{\star}-\langle\sin (2 \theta)\rangle_{\star}^{2}\right\rangle\right\rangle\right\rangle_{\phi} \\
& \frac{1}{\beta K^{2}} \frac{\partial^{2} f_{\mathrm{I}}}{\partial Q_{c c} \partial Q_{c s}}=-4(\beta K)^{2}\left\langle\left\langle\left\langle\langle\sin (2 \theta) \cos (2 \theta)\rangle_{\star}-\langle\sin (2 \theta)\rangle_{\star}\langle\cos (2 \theta)\rangle_{\star}\right\rangle\right\rangle_{\phi}\right. \\
& \frac{1}{\beta K^{2}} \frac{\partial^{2} f_{\mathrm{I}}}{\partial Q_{c c} \partial q_{c c}}=8(\beta K)^{2}\left\langle\left\langle\left\langle\langle\cos (2 \theta) \cos (\theta)\rangle_{\star}\langle\cos (\theta)\rangle_{\star}-\langle\cos (2 \theta)\rangle_{\star}\langle\cos (\theta)\rangle_{\star}^{2}\right\rangle\right\rangle\right\rangle_{\phi} \\
& \frac{1}{\beta K^{2}} \frac{\partial^{2} f_{\mathrm{I}}}{\partial Q_{c c} \partial q_{s s}}=8(\beta K)^{2}\left\langle\left\langle\left\langle\langle\cos (2 \theta) \sin (\theta)\rangle_{\star}\langle\sin (\theta)\rangle_{\star}-\langle\cos (2 \theta)\rangle_{\star}\langle\sin (\theta)\rangle_{\star}^{2}\right\rangle\right\rangle\right\rangle_{\phi} \\
& \frac{1}{\beta K^{2}} \frac{\partial^{2} f_{\mathrm{I}}}{\partial Q_{c c} \partial q_{c s}}=8(\beta K)^{2}\left\langle\left\langle\left\langle\langle\cos (2 \theta) \cos (\theta)\rangle_{\star}\langle\sin (\theta)\rangle_{\star}+\langle\cos (2 \theta) \sin (\theta)\rangle_{\star}\langle\cos (\theta)\rangle_{\star}\right.\right.\right. \\
& \left.\left.-2\langle\cos (2 \theta)\rangle_{\star}\langle\sin (\theta)\rangle_{\star}\langle\cos (\theta)\rangle_{\star}\right\rangle\right\rangle_{\phi} \\
& \frac{1}{\beta K^{2}} \frac{\partial^{2} f_{\mathrm{I}}}{\partial Q_{c s} \partial q_{c c}}=8(\beta K)^{2}\left\langle\left\langle\left\langle\langle\sin (2 \theta) \cos (\theta)\rangle_{\star}\langle\cos (\theta)\rangle_{\star}-\langle\sin (2 \theta)\rangle_{\star}\langle\cos (\theta)\rangle_{\star}^{2}\right\rangle\right\rangle_{\phi}\right. \\
& \frac{1}{\beta K^{2}} \frac{\partial^{2} f_{\mathrm{I}}}{\partial Q_{c s} \partial q_{s s}}=8(\beta K)^{2}\left\langle\left\langle\left\langle\langle\sin (2 \theta) \sin (\theta)\rangle_{\star}\langle\sin (\theta)\rangle_{\star}-\langle\sin (2 \theta)\rangle_{\star}\langle\sin (\theta)\rangle_{\star}^{2}\right\rangle\right\rangle_{\phi}\right. \\
& \frac{1}{\beta K^{2}} \frac{\partial^{2} f_{\mathrm{I}}}{\partial Q_{c s} \partial q_{c s}}=8(\beta K)^{2}\left\langle\left\langle\left\langle\langle\sin (2 \theta) \cos (\theta)\rangle_{\star}\langle\sin (\theta)\rangle_{\star}+\langle\sin (2 \theta) \sin (\theta)\rangle_{\star}\langle\cos (\theta)\rangle_{\star}\right.\right.\right. \\
& \left.\left.\left.-2\langle\sin (2 \theta)\rangle_{\star}\langle\sin (\theta)\rangle_{\star}\langle\cos (\theta)\rangle_{\star}\right\rangle\right\rangle\right\rangle_{\phi} \\
& \frac{1}{\beta K^{2}} \frac{\partial^{2} f_{\mathrm{I}}}{\partial q_{c c}^{2}} \quad=-2+8(\beta K)^{2}\left\langle\left\langle\left\langle\left[\left\langle\cos ^{2}(\theta)\right\rangle_{\star}-\langle\cos (\theta)\rangle_{\star}^{2}\right]\left[\left\langle\cos ^{2}(\theta)\right\rangle_{\star}-3\langle\cos (\theta)\rangle_{\star}^{2}\right]\right\rangle\right\rangle\right\rangle_{\phi} \\
& \frac{1}{\beta K^{2}} \frac{\partial^{2} f_{\mathrm{I}}}{\partial q_{s s}^{2}} \quad=-2+8(\beta K)^{2}\left\langle\left\langle\left\langle\left[\left\langle\sin ^{2}(\theta)\right\rangle_{\star}-\langle\sin (\theta)\rangle_{\star}^{2}\right]\left[\left\langle\sin ^{2}(\theta)\right\rangle_{\star}-3\langle\sin (\theta)\rangle_{\star}^{2}\right]\right\rangle\right\rangle\right\rangle_{\phi} \\
& \frac{1}{\beta K^{2}} \frac{\partial^{2} f_{\mathrm{I}}}{\partial q_{c s}^{2}} \quad=-4+8(\beta K)^{2}\left\langle\left\langle\left\langle\left[\left\langle\sin ^{2}(\theta)\right\rangle_{\star}-\langle\sin (\theta)\rangle_{\star}^{2}\right]\left[\left\langle\cos ^{2}(\theta)\right\rangle_{\star}-3\langle\cos (\theta)\rangle_{\star}^{2}\right]\right.\right.\right. \\
& +\left[\left\langle\cos ^{2}(\theta)\right\rangle_{\star}-\langle\cos (\theta)\rangle_{\star}^{2}\right]\left[\left\langle\sin ^{2}(\theta)\right\rangle_{\star}-3\langle\sin (\theta)\rangle_{\star}^{2}\right] \\
& \left.\left.+2\left[\langle\sin (\theta) \cos (\theta)\rangle_{\star}-\langle\sin (\theta)\rangle_{\star}\langle\cos (\theta)\rangle_{\star}\right]\left[\langle\sin (\theta) \cos (\theta)\rangle_{\star}-3\langle\sin (\theta)\rangle_{\star}\langle\cos (\theta)\rangle_{\star}\right]\right\rangle\right\rangle_{\phi} \\
& \frac{1}{\beta K^{2}} \frac{\partial^{2} f_{\mathrm{I}}}{\partial q_{c c} \partial q_{s s}}=8(\beta K)^{2}\left\langle\left\langle\left\langle\left[\langle\sin (\theta) \cos (\theta)\rangle_{\star}-\langle\sin (\theta)\rangle_{\star}\langle\cos (\theta)\rangle_{\star}\right]\right.\right.\right. \\
& \left.\left.\times\left[\langle\sin (\theta) \cos (\theta)\rangle_{\star}-3\langle\sin (\theta)\rangle_{\star}\langle\cos (\theta)\rangle_{\star}\right]\right\rangle\right\rangle_{\phi} \\
& \frac{1}{\beta K^{2}} \frac{\partial^{2} f_{\mathrm{I}}}{\partial q_{c c} \partial q_{c s}}=8(\beta K)^{2}\left\langle\left\langle\left\langle\left[\langle\sin (\theta) \cos (\theta)\rangle_{\star}-\langle\sin (\theta)\rangle_{\star}\langle\cos (\theta)\rangle_{\star}\right]\left[\left\langle\cos ^{2}(\theta)\right\rangle_{\star}-3\langle\cos (\theta)\rangle_{\star}^{2}\right]\right.\right.\right. \\
& \left.\left.+\left[\langle\sin (\theta) \cos (\theta)\rangle_{\star}-3\langle\sin (\theta)\rangle_{\star}\langle\cos (\theta)\rangle_{\star}\right]\left[\left\langle\cos ^{2}(\theta)\right\rangle_{\star}-\langle\cos (\theta)\rangle_{\star}^{2}\right]\right\rangle\right\rangle_{\phi} \\
& \frac{1}{\beta K^{2}} \frac{\partial^{2} f_{\mathrm{I}}}{\partial q_{s s} \partial q_{c s}}=8(\beta K)^{2}\left\langle\left\langle\left\langle\left[\langle\sin (\theta) \cos (\theta)\rangle_{\star}-\langle\sin (\theta)\rangle_{\star}\langle\cos (\theta)\rangle_{\star}\right]\left[\left\langle\sin ^{2}(\theta)\right\rangle_{\star}-3\langle\sin (\theta)\rangle_{\star}^{2}\right]\right.\right.\right. \\
& \left.\left.\left.+\left[\langle\sin (\theta) \cos (\theta)\rangle_{\star}-3\langle\sin (\theta)\rangle_{\star}\langle\cos (\theta)\rangle_{\star}\right]\left[\left\langle\sin ^{2}(\theta)\right\rangle_{\star}-\langle\sin (\theta)\rangle_{\star}^{2}\right]\right\rangle\right\rangle\right\rangle_{\phi}
\end{aligned}
$$


Frustrated coupled oscillators with random fields - October 23rd, 2002

Appendix A.3. Second order derivatives for model II

$$
\begin{aligned}
& \frac{1}{\beta K^{2}} \frac{\partial^{2} f_{\mathrm{II}}}{\partial Q_{c c}^{2}} \quad=-4-4(\beta K)^{2}\left\langle\left\langle\left\langle\left\langle\cos ^{2}(2 \theta)\right\rangle_{\star}-\langle\cos (2 \theta)\rangle_{\star}^{2}\right\rangle\right\rangle\right\rangle_{\phi} \\
& \frac{1}{\beta K^{2}} \frac{\partial^{2} f_{\mathrm{II}}}{\partial Q_{c s}^{2}} \quad=-4-4(\beta K)^{2}\left\langle\left\langle\left\langle\left\langle\sin ^{2}(2 \theta)\right\rangle_{\star}-\langle\sin (2 \theta)\rangle_{\star}^{2}\right\rangle\right\rangle\right\rangle_{\phi} \\
& \frac{1}{\beta K^{2}} \frac{\partial^{2} f_{\mathrm{II}}}{\partial Q_{c c} \partial Q_{c s}}=-4(\beta K)^{2}\left\langle\left\langle\left\langle\langle\sin (2 \theta) \cos (2 \theta)\rangle_{\star}-\langle\sin (2 \theta)\rangle_{\star}\langle\cos (2 \theta)\rangle_{\star}\right\rangle\right\rangle\right\rangle_{\phi} \\
& \frac{1}{\beta K^{2}} \frac{\partial^{2} f_{\mathrm{II}}}{\partial Q_{c c} \partial q_{c c}}=-8(\beta K)^{2}\left\langle\left\langle\left\langle\langle\cos (2 \theta) \sin (\theta)\rangle_{\star}\langle\sin (\theta)\rangle_{\star}-\langle\cos (2 \theta)\rangle_{\star}\langle\sin (\theta)\rangle_{\star}^{2}\right\rangle\right\rangle\right\rangle_{\phi} \\
& \frac{1}{\beta K^{2}} \frac{\partial^{2} f_{\mathrm{II}}}{\partial Q_{c c} \partial q_{s s}}=-8(\beta K)^{2}\left\langle\left\langle\left\langle\langle\cos (2 \theta) \cos (\theta)\rangle_{\star}\langle\cos (\theta)\rangle_{\star}-\langle\cos (2 \theta)\rangle_{\star}\langle\cos (\theta)\rangle_{\star}^{2}\right\rangle\right\rangle\right\rangle_{\phi} \\
& \frac{1}{\beta K^{2}} \frac{\partial^{2} f_{\mathrm{II}}}{\partial Q_{c c} \partial q_{c s}}=8(\beta K)^{2}\left\langle\left\langle\left\langle\langle\cos (2 \theta) \cos (\theta)\rangle_{\star}\langle\sin (\theta)\rangle_{\star}+\langle\cos (2 \theta) \sin (\theta)\rangle_{\star}\langle\cos (\theta)\rangle_{\star}\right.\right.\right. \\
& \left.\left.\left.-2\langle\cos (2 \theta)\rangle_{\star}\langle\sin (\theta)\rangle_{\star}\langle\cos (\theta)\rangle_{\star}\right\rangle\right\rangle\right\rangle_{\phi} \\
& \frac{1}{\beta K^{2}} \frac{\partial^{2} f_{\mathrm{II}}}{\partial Q_{c s} \partial q_{c c}}=-8(\beta K)^{2}\left\langle\left\langle\left\langle\langle\sin (2 \theta) \sin (\theta)\rangle_{\star}\langle\sin (\theta)\rangle_{\star}-\langle\sin (2 \theta)\rangle_{\star}\langle\sin (\theta)\rangle_{\star}^{2}\right\rangle\right\rangle\right\rangle_{\phi} \\
& \frac{1}{\beta K^{2}} \frac{\partial^{2} f_{\mathrm{II}}}{\partial Q_{c s} \partial q_{s s}}=-8(\beta K)^{2}\left\langle\left\langle\left\langle\langle\sin (2 \theta) \cos (\theta)\rangle_{\star}\langle\cos (\theta)\rangle_{\star}-\langle\sin (2 \theta)\rangle_{\star}\langle\cos (\theta)\rangle_{\star}^{2}\right\rangle\right\rangle\right\rangle_{\phi} \\
& \frac{1}{\beta K^{2}} \frac{\partial^{2} f_{\mathrm{II}}}{\partial Q_{c s} \partial q_{c s}}=8(\beta K)^{2}\left\langle\left\langle\left\langle\langle\sin (2 \theta) \cos (\theta)\rangle_{\star}\langle\sin (\theta)\rangle_{\star}+\langle\sin (2 \theta) \sin (\theta)\rangle_{\star}\langle\cos (\theta)\rangle_{\star}\right.\right.\right. \\
& \left.\left.\left.-2\langle\sin (2 \theta)\rangle_{\star}\langle\sin (\theta)\rangle_{\star}\langle\cos (\theta)\rangle_{\star}\right\rangle\right\rangle\right\rangle_{\phi} \\
& \frac{1}{\beta K^{2}} \frac{\partial^{2} f_{\mathrm{II}}}{\partial q_{c c}^{2}} \quad=8(\beta K)^{2}\left\langle\left\langle\left\langle\left[\left\langle\sin ^{2}(\theta)\right\rangle_{\star}-\langle\sin (\theta)\rangle_{\star}^{2}\right]\left[\left\langle\sin ^{2}(\theta)\right\rangle_{\star}-3\langle\sin (\theta)\rangle_{\star}^{2}\right]\right\rangle\right\rangle\right\rangle_{\phi} \\
& \frac{1}{\beta K^{2}} \frac{\partial^{2} f_{\mathbb{I I}}}{\partial q_{s s}^{2}} \quad=8(\beta K)^{2}\left\langle\left\langle\left\langle\left[\left\langle\cos ^{2}(\theta)\right\rangle_{\star}-\langle\cos (\theta)\rangle_{\star}^{2}\right]\left[\left\langle\cos ^{2}(\theta)\right\rangle_{\star}-3\langle\cos (\theta)\rangle_{\star}^{2}\right]\right\rangle\right\rangle\right\rangle_{\phi} \\
& \frac{1}{\beta K^{2}} \frac{\partial^{2} f_{\mathrm{II}}}{\partial q_{c s}^{2}} \quad=4+8(\beta K)^{2}\left\langle\left\langle\left\langle\left[\left\langle\sin ^{2}(\theta)\right\rangle_{\star}-\langle\sin (\theta)\rangle_{\star}^{2}\right]\left[\left\langle\cos ^{2}(\theta)\right\rangle_{\star}-3\langle\cos (\theta)\rangle_{\star}^{2}\right]\right.\right.\right. \\
& +\left[\left\langle\cos ^{2}(\theta)\right\rangle_{\star}-\langle\cos (\theta)\rangle_{\star}^{2}\right]\left[\left\langle\sin ^{2}(\theta)\right\rangle_{\star}-3\langle\sin (\theta)\rangle_{\star}^{2}\right] \\
& \left.\left.\left.+2\left[\langle\sin (\theta) \cos (\theta)\rangle_{\star}-\langle\sin (\theta)\rangle_{\star}\langle\cos (\theta)\rangle_{\star}\right]\left[\langle\sin (\theta) \cos (\theta)\rangle_{\star}-3\langle\sin (\theta)\rangle_{\star}\langle\cos (\theta)\rangle_{\star}\right]\right\rangle\right\rangle\right\rangle_{\phi} \\
& \frac{1}{\beta K^{2}} \frac{\partial^{2} f_{\mathrm{II}}}{\partial q_{c c} \partial q_{s s}}=-2+8(\beta K)^{2}\left\langle\left\langle\left\langle\left[\langle\sin (\theta) \cos (\theta)\rangle_{\star}-\langle\sin (\theta)\rangle_{\star}\langle\cos (\theta)\rangle_{\star}\right]\right.\right.\right. \\
& \left.\left.\left.\times\left[\langle\sin (\theta) \cos (\theta)\rangle_{\star}-3\langle\sin (\theta)\rangle_{\star}\langle\cos (\theta)\rangle_{\star}\right]\right\rangle\right\rangle\right\rangle_{\phi} \\
& \frac{1}{\beta K^{2}} \frac{\partial^{2} f_{\mathrm{II}}}{\partial q_{c c} \partial q_{c s}}=-8(\beta K)^{2}\left\langle\left\langle\left\langle\left[\langle\sin (\theta) \cos (\theta)\rangle_{\star}-\langle\sin (\theta)\rangle_{\star}\langle\cos (\theta)\rangle_{\star}\right]\left[\left\langle\sin ^{2}(\theta)\right\rangle_{\star}-3\langle\sin (\theta)\rangle_{\star}^{2}\right]\right.\right.\right. \\
& \left.\left.\left.+\left[\langle\sin (\theta) \cos (\theta)\rangle_{\star}-3\langle\sin (\theta)\rangle_{\star}\langle\cos (\theta)\rangle_{\star}\right]\left[\left\langle\sin ^{2}(\theta)\right\rangle_{\star}-\langle\sin (\theta)\rangle_{\star}^{2}\right]\right\rangle\right\rangle\right\rangle_{\phi} \\
& \frac{1}{\beta K^{2}} \frac{\partial^{2} f_{\mathrm{II}}}{\partial q_{s s} \partial q_{c s}}=-8(\beta K)^{2}\left\langle\left\langle\left\langle\left[\langle\sin (\theta) \cos (\theta)\rangle_{\star}-\langle\sin (\theta)\rangle_{\star}\langle\cos (\theta)\rangle_{\star}\right]\left[\left\langle\cos ^{2}(\theta)\right\rangle_{\star}-3\langle\cos (\theta)\rangle_{\star}^{2}\right]\right.\right.\right. \\
& \left.\left.\left.+\left[\langle\sin (\theta) \cos (\theta)\rangle_{\star}-3\langle\sin (\theta)\rangle_{\star}\langle\cos (\theta)\rangle_{\star}\right]\left[\left\langle\cos ^{2}(\theta)\right\rangle_{\star}-\langle\cos (\theta)\rangle_{\star}^{2}\right]\right\rangle\right\rangle\right\rangle_{\phi}
\end{aligned}
$$




\section{Appendix B. Derivation of the AT Instability}

\section{Appendix B.1. Calculation of the RS Hessian for replicon fluctuations}

We calculate the Hessian of the disorder-averaged free energy per oscillator by expanding (9) in powers of the fluctuations $\left\{\delta \boldsymbol{q}^{\star \star}, \delta \hat{\boldsymbol{q}}^{\star \star}\right\}$ around the replica-symmetric saddle-point. In this appendix we will use pairs of Roman indices $(a b)$ and $(d e)$ to label the four combinations $\{c c, s s, c s, s c\}$, and abbreviate the corresponding functions in the obvious way as $a(\theta), b(\theta), c(\theta), d(\theta) \in\{\cos (\theta), \sin (\theta)\}$. We put $\hat{q}_{\alpha \beta}^{\star \star}=2 i(\beta K)^{2} k_{\alpha \beta}^{\star \star}$ and $\delta \bar{f}[\ldots]=\bar{f}[\ldots]-\bar{f}_{\mathrm{RS}}[\ldots]$, and obtain (since linear terms are absent):

$$
\begin{aligned}
& -\frac{n}{\beta K^{2}} \delta \bar{f}[\ldots]=2 \sin ^{2}\left(A^{\star}\right) \sum_{\alpha \beta}\left[\delta q_{\alpha \beta}^{s s} \delta q_{\alpha \beta}^{c c}-\delta q_{\alpha \beta}^{s c} \delta q_{\alpha \beta}^{c s}\right]+\cos ^{2}\left(A^{\star}\right) \sum_{a b} \sum_{\alpha \beta}\left[\delta q_{\alpha \beta}^{a b}\right]^{2} \\
& -2 \sum_{a b} \sum_{\alpha \beta} \delta k_{\alpha \beta}^{a b} \delta q_{\alpha \beta}^{a b}+\frac{1}{(\beta K)^{2}}\left\langle\log \left\{\frac{\int d \boldsymbol{\theta} M\left(\boldsymbol{\theta} \mid\left\{\boldsymbol{k}^{\star \star}+\delta \boldsymbol{k}^{\star \star}\right\}\right)}{\int d \boldsymbol{\theta} M\left(\boldsymbol{\theta} \mid\left\{\boldsymbol{k}^{\star \star}\right\}\right)}\right\}\right\rangle_{\phi}+\ldots
\end{aligned}
$$

with

$$
M\left(\boldsymbol{\theta} \mid\left\{\boldsymbol{k}^{\star \star}\right\}\right)=e^{\beta h \sum_{\alpha} \cos \left(\theta^{\alpha}-\phi\right)+2(\beta K)^{2} \sum_{a b} \sum_{\alpha \beta} k_{\alpha \beta}^{a b} a\left(\theta_{\alpha}\right) b\left(\theta_{\beta}\right)}
$$

Working out the fraction in the last term of (B.1) gives

$$
\begin{aligned}
& \frac{\int d \boldsymbol{\theta} M\left(\boldsymbol{\theta} \mid\left\{\boldsymbol{k}^{\star \star}+\delta \boldsymbol{k}^{\star \star}\right\}\right)}{\int d \boldsymbol{\theta} M\left(\boldsymbol{\theta} \mid\left\{\boldsymbol{k}^{\star \star}\right\}\right)}=1+2(\beta K)^{2} \sum_{\alpha \beta} \sum_{a b} \delta k_{\alpha \beta}^{a b} \frac{\int d \boldsymbol{\theta} M\left(\boldsymbol{\theta} \mid\left\{\boldsymbol{k}^{\star \star}\right\}\right) a\left(\theta_{\alpha}\right) b\left(\theta_{\beta}\right)}{\int d \boldsymbol{\theta} M\left(\boldsymbol{\theta} \mid\left\{\boldsymbol{k}^{\star \star}\right\}\right)} \\
& +2(\beta K)^{4} \sum_{\alpha \beta \gamma \delta} \sum_{a b, d e} \delta k_{\alpha \beta}^{a b} \delta k_{\gamma \delta}^{d e} \frac{\int d \boldsymbol{\theta} M\left(\boldsymbol{\theta} \mid\left\{\boldsymbol{k}^{\star \star}\right\}\right) a\left(\theta_{\alpha}\right) b\left(\theta_{\beta}\right) d\left(\theta_{\gamma}\right) e\left(\theta_{\delta}\right)}{\int d \boldsymbol{\theta} M\left(\boldsymbol{\theta} \mid\left\{\boldsymbol{k}^{\star \star}\right\}\right)}+\ldots
\end{aligned}
$$

Thus, upon expanding $\log (x)=x-\frac{1}{2} x^{2}+\ldots$, and upon introducing the short-hand $M(\boldsymbol{\theta} \mid \mathrm{RS})=M\left(\boldsymbol{\theta} \mid\left\{\boldsymbol{k}_{\mathrm{RS}}^{\star \star}\right\}\right)$, we arrive at

$$
\begin{gathered}
-\frac{n}{\beta K^{2}} \delta \bar{f}[\ldots]=2 \sin ^{2}\left(A^{\star}\right) \sum_{\alpha \beta}\left[\delta q_{\alpha \beta}^{s s} \delta q_{\alpha \beta}^{c c}-\delta q_{\alpha \beta}^{s c} \delta q_{\alpha \beta}^{c s}\right]+\cos ^{2}\left(A^{\star}\right) \sum_{a b} \sum_{\alpha \beta}\left[\delta q_{\alpha \beta}^{a b}\right]^{2} \\
-2 \sum_{a b} \sum_{\alpha \beta} \delta k_{\alpha \beta}^{a b} \delta q_{\alpha \beta}^{a b}+2(\beta K)^{2} \sum_{\alpha \beta \gamma \delta} \sum_{a b, d e} \delta k_{\alpha \beta}^{a b} \delta k_{\gamma \delta}^{d e}\left\langle\frac{\int d \boldsymbol{\theta} M(\boldsymbol{\theta} \mid \mathrm{RS}) a\left(\theta_{\alpha}\right) b\left(\theta_{\beta}\right) d\left(\theta_{\gamma}\right) e\left(\theta_{\delta}\right)}{\int d \boldsymbol{\theta} M(\boldsymbol{\theta} \mid \mathrm{RS})}\right. \\
\left.-\frac{\int d \boldsymbol{\theta} M(\boldsymbol{\theta} \mid \mathrm{RS}) a\left(\theta_{\alpha}\right) b\left(\theta_{\beta}\right)}{\int d \boldsymbol{\theta} M(\boldsymbol{\theta} \mid \mathrm{RS})} \frac{\int d \boldsymbol{\theta} M(\boldsymbol{\theta} \mid \mathrm{RS}) d\left(\theta_{\gamma}\right) e\left(\theta_{\delta}\right)}{\int d \boldsymbol{\theta} M(\boldsymbol{\theta} \mid \mathrm{RS})}\right\rangle_{\phi}+\ldots
\end{gathered}
$$

We next work out the last of the above quadratic terms for fluctuations around the RS solution (19), for small $n$. We use the short-hand Dxyuv $=D x D y D u D v$, and denote by $M(\theta)$ either the measure (26) (for model I) or 27) (for model II):

$$
\begin{aligned}
& \langle\ldots\rangle_{\phi}=\left\langle\frac{\int D x y u v\left[\prod_{\lambda} \int d \theta M\left(\theta_{\lambda}\right)\right] a\left(\theta_{\alpha}\right) b\left(\theta_{\beta}\right) c\left(\theta_{\gamma}\right) d\left(\theta_{\delta}\right)}{\int D x y u v\left[\int d \theta M(\theta)\right]^{n}}\right\rangle_{\phi} \\
& \quad-\left\langle\frac{\int D x y u v\left[\prod_{\lambda} \int d \theta_{\lambda} M\left(\theta_{\lambda}\right)\right] a\left(\theta_{\alpha}\right) b\left(\theta_{\beta}\right)}{\int D x y u v\left[\int d \theta M(\theta)\right]^{n}} \frac{\int D x y u v\left[\prod_{\lambda} \int d \theta_{\lambda} M\left(\theta_{\lambda}\right)\right] c\left(\theta_{\gamma}\right) d\left(\theta_{\delta}\right)}{\int D x y u v\left[\int d \theta M(\theta)\right]^{n}}\right\rangle_{\phi}
\end{aligned}
$$

If we restrict ourselves to replicon fluctuations, where $\delta q_{\alpha \alpha}^{\star \star}=\delta k_{\alpha \alpha}^{\star \star}=0, \sum_{\alpha} \delta k_{\alpha \beta}=$ $\sum_{\alpha} \delta q_{\alpha \beta}=0$ and $\sum_{\beta} \delta k_{\alpha \beta}=\sum_{\beta} \delta q_{\alpha \beta}=0$, we can proceed by inserting a string of Kronecker symbols (and complementary symbols $\bar{\delta}_{\alpha \beta}=1-\delta_{\alpha \beta}$ ) to streamline the bookkeeping of possibly identical combinations of replica indices:

$$
\delta_{\alpha \delta} \delta_{\beta \gamma}+\delta_{\alpha \gamma} \delta_{\beta \delta}+\delta_{\alpha \delta} \bar{\delta}_{\beta \gamma}+\delta_{\alpha \gamma} \bar{\delta}_{\beta \delta}+\bar{\delta}_{\alpha \gamma} \delta_{\beta \delta}+\bar{\delta}_{\alpha \delta} \delta_{\beta \gamma}+\bar{\delta}_{\alpha \delta} \bar{\delta}_{\alpha \gamma} \bar{\delta}_{\beta \delta} \bar{\delta}_{\beta \gamma}
$$


giving, for $n \rightarrow 0$, and upon using again the replicon properties which allow us to drop contributions which will not survive the summation over $(\alpha, \beta ; a b)$ and $(\gamma, \delta ; d e)$ :

$$
\begin{aligned}
\langle\ldots\rangle_{\phi} & =\delta_{\alpha \delta} \delta_{\beta \gamma}\left\langle\left\langle\left\langle\left[\langle a(\theta) e(\theta)\rangle_{\star}-\langle a(\theta)\rangle_{\star}\langle e(\theta)\rangle_{\star}\right]\left[\langle b(\theta) d(\theta)\rangle_{\star}-\langle b(\theta)\rangle_{\star}\langle d(\theta)\rangle_{\star}\right]\right\rangle\right\rangle_{\phi}\right. \\
& +\delta_{\beta \delta} \delta_{\alpha \gamma}\left\langle\left\langle\left\langle\left[\langle b(\theta) e(\theta)\rangle_{\star}-\langle b(\theta)\rangle_{\star}\langle e(\theta)\rangle_{\star}\right]\left[\langle a(\theta) d(\theta)\rangle_{\star}-\langle a(\theta)\rangle_{\star}\langle d(\theta)\rangle_{\star}\right]\right\rangle\right\rangle_{\phi}\right.
\end{aligned}
$$

These two remaining terms will give identical contributions to the fluctuations around the RS free energy, after the summation over $(\gamma, \delta ; d e)$ has been carried out, and the result can be written in the compact form

$$
\begin{aligned}
-\frac{n}{\beta K^{2}} \delta \bar{f}[\ldots]= & \sum_{\alpha \neq \beta} \sum_{a b, d e}\left\{\delta q_{\alpha \beta}^{a b} \delta q_{\alpha \beta}^{d e}\left[\sin ^{2}\left(A^{\star}\right) C_{a b, d e}+\cos ^{2}\left(A^{\star}\right) \delta_{a b, d e}\right]\right. \\
& \left.-2 \delta q_{\alpha \beta} \delta k_{\alpha \beta}^{d e} \delta_{a b, d e}+4(\beta K)^{2} \delta k_{\alpha \beta}^{a b} \delta k_{\alpha \beta}^{d e} E_{a b, d e}\right\}+\ldots
\end{aligned}
$$

with the two $4 \times 4$ matrices (note: $a b, d e \in\{c c, s s, c s, s c\}$ )

$$
\begin{aligned}
C_{a b, d e} & =\delta_{a b, s s} \delta_{d e, c c}+\delta_{a b, c c} \delta_{d e, s s}-\delta_{a b, c s} \delta_{d e, s c}-\delta_{a b, s c} \delta_{d e, c s} \\
E_{a b, d e} & =\left\langle\left\langle\left\langle[b(\theta) e(\theta)\rangle_{\star}-\langle b(\theta)\rangle_{\star}\langle e(\theta)\rangle_{\star}\right]\left[\langle a(\theta) d(\theta)\rangle_{\star}-\langle a(\theta)\rangle_{\star}\langle d(\theta)\rangle_{\star}\right]\right\rangle\right\rangle_{\phi}
\end{aligned}
$$

Expression (B.2) shows that the replica indices $(\alpha, \beta)$ have become irrelevant labels, and that for $n \rightarrow 0$ and within the sub-space of replicon fluctuations $\left\{\delta k_{\alpha \beta}^{\star \star}, \delta q_{\alpha \beta}^{\star \star}\right\}$, the spectrum of the Hessian reduces to that of the following $8 \times 8$ matrix:

$$
\mathcal{H}=\left(\begin{array}{cc}
\cos ^{2}\left(A^{\star}\right) \mathbf{I}+\sin ^{2}\left(A^{\star}\right) \boldsymbol{C} & -\mathbf{I} \\
-\mathbf{I} & 4(\beta K)^{2} \boldsymbol{E}
\end{array}\right)
$$

(apart from an overall multiplicative constant), with the building blocks $\boldsymbol{C}=\left\{C_{a b, d e}\right\}$ and $\boldsymbol{E}=\left\{E_{a b, d e}\right\}$ as given in (B.3,B.4 and with the $4 \times 4$ unit matrix $\mathbf{\mathbf { I }}_{a b, d e}=\delta_{a b, d e}$.

\section{Appendix B.2. Replicon instabilities}

Requiring the Hessian to have a zero eigenvalue (replicon instability), using the above results, leads us to the following condition:

$$
\begin{array}{ll}
\exists\left(\begin{array}{l}
\boldsymbol{x} \\
\boldsymbol{y}
\end{array}\right) \neq\left(\begin{array}{l}
\mathbf{0} \\
\mathbf{0}
\end{array}\right): \quad & \left(\begin{array}{cc}
\cos ^{2}\left(A^{\star}\right) \mathbf{I}+\sin ^{2}\left(A^{\star}\right) \boldsymbol{C} & -\mathbf{I} \\
-\mathbf{I} & 4(\beta K) 2 \boldsymbol{E}
\end{array}\right)\left(\begin{array}{l}
\boldsymbol{x} \\
\boldsymbol{y}
\end{array}\right)=\left(\begin{array}{l}
\mathbf{0} \\
\mathbf{0}
\end{array}\right) \\
\exists\left(\begin{array}{l}
\boldsymbol{x} \\
\boldsymbol{y}
\end{array}\right) \neq\left(\begin{array}{l}
\mathbf{0} \\
\mathbf{0}
\end{array}\right): \quad\left\{\begin{array}{l}
{\left[\cos ^{2}\left(A^{\star}\right) \mathbf{I}+\sin ^{2}\left(A^{\star}\right) \boldsymbol{C}\right] \boldsymbol{x}=\boldsymbol{y}} \\
\boldsymbol{x}=4(\beta K)^{2} \boldsymbol{E} \boldsymbol{y}
\end{array}\right.
\end{array}
$$

Equivalently:

$$
\operatorname{Det}\left\{4(\beta K)^{2}\left[\cos ^{2}\left(A^{\star}\right) \mathbf{I}+\sin ^{2}\left(A^{\star}\right) \boldsymbol{C}\right] \boldsymbol{E}-\mathbf{I}\right\}=0
$$

For our two models I (where $A^{\star}=0$ ) and II (where $A^{\star}=\frac{1}{2} \pi$ ) this translates into

$$
\begin{array}{ll}
\text { Model I : } & \operatorname{Det}\left[\boldsymbol{E}-(T / 2 K)^{2} \mathbf{I}\right]=0 \\
\text { Model II : } & \operatorname{Det}\left[\boldsymbol{E}-(T / 2 K)^{2} \boldsymbol{C}\right]=0
\end{array}
$$

where we have used the property $C^{2}=\mathbf{I}$. 
In the representation where the various entries of the matrices are ordered as $\{c c, s s, c s, s c\}$, and with assistance of the abbreviations (28,28), we find our matrices $\boldsymbol{C}$ and $\boldsymbol{E}$ (which will generally not commute) to acquire the following form:

$$
\boldsymbol{C}=\left(\begin{array}{cccc}
0 & 1 & 0 & 0 \\
1 & 0 & 0 & 0 \\
0 & 0 & 0 & -1 \\
0 & 0 & -1 & 0
\end{array}\right) \quad \boldsymbol{E}=\left\langle\left\langle\left\langle\left(\begin{array}{cccc}
\gamma_{c c}^{2} & \gamma_{c s}^{2} & \gamma_{c c} \gamma_{c s} & \gamma_{c c} \gamma_{c s} \\
\gamma_{c s}^{2} & \gamma_{s s}^{2} & \gamma_{s s} \gamma_{c s} & \gamma_{s s} \gamma_{c s} \\
\gamma_{c c} \gamma_{c s} & \gamma_{s s} \gamma_{c s} & \gamma_{c c} \gamma_{s s} & \gamma_{c s}^{2} \\
\gamma_{c c} \gamma_{c s} & \gamma_{s s} \gamma_{c s} & \gamma_{c s}^{2} & \gamma_{c c} \gamma_{s s}
\end{array}\right)\right\rangle\right\rangle_{\phi}\right.
$$

In the representation (B.8) one observes that the vector $|0\rangle=\frac{1}{2} \sqrt{2}(0,0,1,-1)$ is an eigenvector of both $\boldsymbol{C}$ (with eigenvalue 1) and $\boldsymbol{E}$ (with eigenvalue $\left\langle\left\langle\left\langle\gamma_{c c} \gamma_{s s}-\gamma_{c s}^{2}\right\rangle\right\rangle\right\rangle_{\phi}$ ), and therefore also of the two matrices in (B.6. B.7). This immediately leads us to the first replicon instability condition:

$$
(T / 2 K)^{2}=\left\langle\left\langle\left\langle\gamma_{c c} \gamma_{s s}-\gamma_{c s}^{2}\right\rangle\right\rangle\right\rangle_{\phi}
$$

(for both models I and II).

The remaining three eigenvalues of the relevant matrices in (B.6.B.7), viz. $\boldsymbol{E}-(T / 2 K)^{2} \mathbf{I}$ (for model I) and $\boldsymbol{E}-(T / 2 K)^{2} \boldsymbol{C}$ (for model II), must all be orthogonal to $|0\rangle$, and are thus in the sub-space spanned by the following three orthogonal eigenvectors of $\boldsymbol{C}$ (with eigenvalues $\{1,-1,-1\}$, respectively):

$$
|1\rangle=\frac{1}{2} \sqrt{2}(1,1,0,0), \quad|2\rangle=\frac{1}{2} \sqrt{2}(1,-1,0,0), \quad|3\rangle=\frac{1}{2} \sqrt{2}(0,0,1,1)
$$

After some simple algebra one finds that on the basis $\{|1\rangle,|2\rangle,|3\rangle\}$ the matrices (B.8) reduce to the following $3 \times 3$ ones

$$
\begin{gathered}
\boldsymbol{C}=\left(\begin{array}{ccc}
1 & 0 & 0 \\
0 & -1 & 0 \\
0 & 0 & -1
\end{array}\right) \\
\boldsymbol{E}=\left(\begin{array}{ccc}
\left\langle\left\langle\left\langle\frac{1}{2}\left(\gamma_{c c}^{2}+\gamma_{s s}^{2}\right)+\gamma_{c s}^{2}\right\rangle\right\rangle\right\rangle_{\phi} & \left\langle\left\langle\left\langle\frac{1}{2}\left(\gamma_{c c}^{2}-\gamma_{s s}^{2}\right)\right\rangle\right\rangle_{\phi}\right. & \left\langle\left\langle\left\langle\gamma_{c s}\left(\gamma_{c c}+\gamma_{s s}\right)\right\rangle\right\rangle\right\rangle_{\phi} \\
\left\langle\left\langle\left\langle\frac{1}{2}\left(\gamma_{c c}^{2}-\gamma_{s s}^{2}\right)\right\rangle\right\rangle\right\rangle_{\phi} & \left\langle\left\langle\left\langle\frac{1}{2}\left(\gamma_{c c}^{2}+\gamma_{s s}^{2}\right)-\gamma_{c s}^{2}\right\rangle\right\rangle\right\rangle_{\phi} & \left\langle\left\langle\left\langle\gamma_{c s}\left(\gamma_{c c}-\gamma_{s s}\right)\right\rangle\right\rangle_{\phi}\right. \\
\left\langle\left\langle\left\langle\gamma_{c s}\left(\gamma_{c c}+\gamma_{s s}\right)\right\rangle\right\rangle\right\rangle_{\phi} & \left\langle\left\langle\left\langle\gamma_{c s}\left(\gamma_{c c}-\gamma_{s s}\right)\right\rangle\right\rangle_{\phi}\right. & \left\langle\left\langle\left\langle\gamma_{c c} \gamma_{s s}+\gamma_{c s}^{2}\right\rangle\right\rangle_{\phi}\right.
\end{array}\right)
\end{gathered}
$$

The remaining replicon instabilities now follow upon inserting (B.10, B.11) into the conditions (B.6. B.7). The physical RSB transition associated with the combined replicon instabilities is the one occurring at the highest temperature. 